\title{
ON SOLVABLE SPHERICAL SUBGROUPS OF SEMISIMPLE ALGEBRAIC GROUPS
}

\author{
R. S. AVDEEV
}

\begin{abstract}
We develop a structure theory of connected solvable spherical subgroups in semisimple algebraic groups. Based on this theory, we obtain an explicit classification of all such subgroups up to conjugacy.
\end{abstract}

\section{$\S 1$. INTRODUCTION}

1.1. Let $G$ be a connected semisimple complex algebraic group. A closed subgroup $H \subset G$ (resp. a homogeneous space $G / H$ ) is said to be spherical if one of the following three equivalent conditions holds:

(1) a Borel subgroup $B \subset G$ has an open orbit in $G / H$;

(2) every irreducible $G$-variety $X$ containing $G / H$ as an open orbit has finitely many $G$-orbits;

(3) for every irreducible finite-dimensional $G$-module $V$ and every character $\chi$ of $H$, the dimension of the subspace $\{v \in V \mid h v=\chi(h) v \forall h \in H\} \subset V$ is at most 1 .

There are other characterizations of spherical subgroups, but the three given above are most often used to study them.

Spherical homogeneous spaces have been studied intensively over the last thirty years. However, the problem of classifying these spaces or, equivalently, classifying spherical subgroups in semisimple algebraic groups still remains of importance. Let us give a brief historical account of this question. The first significant result in this direction was obtained by Krämer [1] in 1979. He classified all reductive spherical subgroups in simple groups. Then Mikityuk [2] in 1986 and, independently, Brion [3] in 1987 classified all reductive spherical subgroups in arbitrary semisimple groups (see also [4] for a more accurate formulation). The next step towards a classification of spherical homogeneous spaces was Luna's 1993 preprint [5], where solvable spherical subgroups in semisimple groups were considered. In this preprint, under certain restrictions, all such subgroups were described in the following sense: with each subgroup one associates a set of combinatorial data that uniquely determines this subgroup, and then one classifies all sets that can appear in this way. In 2001 Luna 6] created a theory of spherical systems and, using this theory, described (in the same sense) all spherical subgroups in semisimple groups of type A. During the following several years Luna's approach was applied successfully by Bravi and Pezzini to certain other types of semisimple groups, including all the classical groups (for details, the reader is referred to the paper [7] and its bibliography). Finally, in 2009 a new approach to the problem was proposed by CupitFoutou who completed the proof of the so-called Luna conjecture and thereby obtained a description of all spherical subgroups in arbitrary semisimple groups [8]. Thus, at

2010 Mathematics Subject Classification. Primary 20G07; Secondary 14M27, 14 M17.

Key words and phrases. Algebraic group, homogeneous space, spherical subgroup, solvable subgroup.

This research was partially supported by the Russian Foundation for Basic Research (grant no. 0901-00648-a). 
this moment there is a description in combinatorial terms of all spherical subgroups in semisimple groups. However, this description has the following shortcoming: it does not provide a simple method for constructing a spherical subgroup from the set of invariants that determines this subgroup, even in the case of solvable spherical subgroups. In other words, the existing description is implicit. Because of this, it is still of interest to obtain an explicit classification of all spherical subgroups in semisimple groups.

This paper contains a new approach to the classification of connected solvable spherical subgroups in semisimple algebraic groups. This approach is radically different from the approach Luna [5] used in 1993 and provides an explicit classification. We note that the above-mentioned results of Luna and the others are not used in this paper.

1.2. Throughout the paper, the ground field is the field $\mathbb{C}$ of complex numbers. All topological terms relate to the Zariski topology. All groups are assumed to be algebraic and their subgroups closed. The tangent algebras of groups denoted by capital Latin letters are denoted by the corresponding small German letters. Weights of tori are identified with their differentials.

Until the end of the paper we fix the following notation:

$G$ is an arbitrary connected semisimple algebraic group;

$B \subset G$ is a fixed Borel subgroup of $G$;

$T \subset B$ is a fixed maximal torus of $G$;

$U \subset B$ is the maximal unipotent subgroup of $G$ contained in $B$;

$N_{G}(T)$ is the normalizer of $T$ in $G$;

$W=N_{G}(T) / T$ is the Weyl group of $G$ with respect to $T$;

$\mathfrak{X}(T)$ is the character lattice (weight lattice) of $T$;

$Q=\mathfrak{X}(T) \otimes_{\mathbb{Z}} \mathbb{Q}$ is the rational vector space spanned by $\mathfrak{X}(T)$;

$(\cdot, \cdot)$ is a fixed inner product in $Q$ invariant with respect to $W$;

$\Delta \subset \mathfrak{X}(T)$ is the root system of $G$ with respect to $T$;

$\Delta_{+} \subset \Delta$ is the subset of positive roots with respect to $B$;

$\Pi \subset \Delta_{+}$is the set of simple roots;

$r_{\alpha} \in W$ is the simple reflection corresponding to a root $\alpha \in \Pi$;

$\bar{w} \in N_{G}(T)$ is a fixed representative of an element $w \in W$;

$\mathfrak{g}_{\alpha} \subset \mathfrak{g}$ is the root subspace corresponding to a root $\alpha \in \Delta$;

$e_{\alpha} \in \mathfrak{g}_{\alpha}$ is a fixed nonzero element.

Let $H \subset B$ be a connected solvable subgroup, and $N \subset U$ its unipotent radical. We say that $H$ is standardly embedded in $B$ (with respect to $T$ ) if the subgroup $S=H \cap T \subset T$ is a maximal torus in $H$. In this situation, we obviously have $H=S \curlywedge N$. It is well known that every connected solvable subgroup of $G$ is conjugate by a suitable element of $G$ to a subgroup that is standardly embedded in $B$.

1.3. We now discuss the structure of our paper and its main ideas.

In $\S 2$, we prove a convenient criterion for sphericity of a connected solvable subgroup in terms of its tangent algebra (Theorem 11). This criterion is the foundation of the entire paper. We then use this criterion to prove Theorem 2 which can be regarded as a first approximation to the classification of connected solvable spherical subgroups. Theorem 2 asserts that a connected solvable spherical subgroup $H$ standardly embedded in $B$ is uniquely determined by its maximal torus $S=H \cap T$ together with the set $\Psi=\left\{\alpha \in \Delta_{+} \mid \mathfrak{g}_{\alpha} \not \subset \mathfrak{h}\right\} \subset \Delta_{+}$.

In $\S 3$ we investigate the question of what kind of set the set $\Psi$ can be. The term "active roots" is introduced for roots in this set. Having studied properties of a single active root in relation to the others we list all positive roots that can be elements of $\Psi$ depending on the root system $\Delta$ (Theorem 3). As a result of further analysis of active 
roots, with every connected solvable spherical subgroup $H$ standardly embedded in $B$ we associate a set of combinatorial data $\Upsilon(H)=(S, \mathrm{M}, \pi, \sim)$, where $S=H \cap T$ is a maximal torus in $H, \mathrm{M} \subset \Psi$ is the set of so-called maximal active roots, $\pi: \mathrm{M} \rightarrow \Pi$ is a map, and $\sim$ is an equivalence relation on $\mathrm{M}$. Then we determine a number of conditions that are satisfied by the set $\Upsilon(H)$. The uniqueness theorem (Theorem 4) concludes the section: every connected solvable spherical subgroup $H$ standardly embedded in $B$ is uniquely determined by its set of combinatorial data $\Upsilon(H)$.

In $\S$ 4 we prove the existence theorem (Theorem [5): for every abstract set of combinatorial data $(S, \mathrm{M}, \pi, \sim)$ satisfying the conditions listed in the uniqueness theorem, there exists a connected solvable spherical subgroup $H$ standardly embedded in $B$ with this set of combinatorial data. The proof of the existence theorem contains an algorithm for constructing the subgroup $H$ from the set $(S, \mathrm{M}, \pi, \sim)$.

Section 5 is devoted to the question of when two connected solvable spherical subgroups standardly embedded in $B$ are conjugate in $G$. With this goal in mind, we introduce the notion of an elementary transformation. An elementary transformation is a transition of the form $H_{1} \mapsto H_{2}$, where $H_{1}, H_{2}$ are connected solvable spherical subgroups standardly embedded in $B$ and $H_{2}=\sigma_{\alpha} H_{1} \sigma_{\alpha}^{-1}$ for some representative $\sigma_{\alpha} \in N_{G}(T)$ of the simple reflection $r_{\alpha}$. The answer to the question under consideration is given by Theorem 6. two connected solvable spherical subgroups standardly embedded in $B$ are conjugate in $G$ if and only if there exists a chain of elementary transformations taking one of these subgroups to the other. Theorems 4, 5, and 6 already give a complete classification of connected solvable spherical subgroups in semisimple groups. Further, in the context of the general theory, we consider an important special case of connected solvable spherical subgroups in more detail, namely, the case of subgroups having finite index in their normalizers. Compared with the general case, the classification of such subgroups is reformulated in a simpler form.

In $\S 6$ we show that the conjugacy class of every connected solvable spherical subgroup contains a subgroup $H$ standardly embedded in $B$ such that the set $\Upsilon(H)$ satisfies stronger conditions than those stated in the uniqueness theorem (Theorem 7 ). We call such sets $\Upsilon(H)$ "reduced". Then we prove that for any two connected solvable spherical subgroups standardly embedded in $B$ and conjugate in $G$ such that their sets of combinatorial data are reduced, there exists a chain of elementary transformations taking one of these subgroups to the other such that every intermediate subgroup of this chain also has a reduced set of combinatorial data (Theorem 8).

Finally, $\S[7$ contains examples where we apply the theory developed in this paper. Namely, in this section, in all simple groups $G$ of rank at most 4 we list, up to conjugacy, all connected solvable spherical subgroups having finite index in their normalizers. The results of $\S 6$ play a considerable role in simplifying the enumeration procedure.

The main results of this paper were announced at the workshop "Algebraic Groups", Oberwolfach, Germany, 18-24 April, 2010 (see 9]).

\subsection{Some notation and conventions.}

$e$ is the identity element of an arbitrary group;

$|X|$ is the cardinality of a finite set $X$;

$\langle A\rangle$ is the linear hull of a subset $A \subset \mathfrak{X}(T)$ in the space $Q$;

$V^{*}$ is the space of linear functions on a vector space $V$;

$Z_{L}(K)$ is the centralizer of a subgroup $K$ in a group $L$;

$N_{L}(K)$ is the normalizer of a subgroup $K$ in a group $L$;

$L^{0}$ is the connected component of the identity of a group $L$;

$\mathfrak{X}(L)$ is the group of characters (in additive notation) of a group $L$;

$\operatorname{rk} L$ is the rank of a reductive group $L$, that is, the dimension of a maximal torus in $L$; 
$\Sigma(\widetilde{\Pi})$ is the Dynkin diagram of a subset $\widetilde{\Pi} \subset \Pi$.

For every root $\alpha=\sum_{\gamma \in \Pi} k_{\gamma} \gamma \in \Delta_{+}$, we introduce its support $\operatorname{Supp} \alpha=\left\{\gamma \mid k_{\gamma}>0\right\}$ and height ht $\alpha=\sum_{\gamma \in \Pi} k_{\gamma}$. If $\alpha \in \Delta_{+}$, then we set $\Delta(\alpha)=\Delta \cap\langle\operatorname{Supp} \alpha\rangle$ and $\Delta_{+}(\alpha)=$ $\Delta_{+} \cap\langle\operatorname{Supp} \alpha\rangle$. The set $\Delta(\alpha)$ is an indecomposable root system whose set of simple roots is $\operatorname{Supp} \alpha$. The set of positive roots of $\Delta(\alpha)$ coincides with $\Delta_{+}(\alpha)$.

Let $L$ be a group, and $L_{1}, L_{2}$ subgroups of it. Then we write $L=L_{1} \wedge L_{2}$ to indicate that $L$ decomposes into a semidirect product of $L_{1}, L_{2}$, that is, $L=L_{1} L_{2}, L_{1} \cap L_{2}=\{e\}$, and $L_{2}$ is a normal subgroup of $L$.

Abusing the language, we identify roots in $\Pi$ and the corresponding nodes of the Dynkin diagram of $\Pi$.

If we say that two nodes of a Dynkin diagram are connected by an edge we allow this edge to be multiple.

For connected Dynkin diagrams, we use the same numeration of simple roots as in the book [10].

\section{$\S 2$. SPHERICITY CRITERION AND SOME APPLICATIONS}

2.1. Let $H \subset G$ be a fixed connected solvable subgroup standardly embedded in $B$. Let $S=H \cap T$ and $N=H \cap U$ be a maximal torus and the unipotent radical of $H$, respectively. We denote by $\tau: \mathfrak{X}(T) \rightarrow \mathfrak{X}(S)$ the character restriction map from $T$ to $S$. Let $\Phi=\tau\left(\Delta_{+}\right) \subset \mathfrak{X}(S)$ be the weight system of the natural action of $S$ on $\mathfrak{u}$. We have $\mathfrak{u}=\bigoplus_{\lambda \in \Phi} \mathfrak{u}_{\lambda}$, where $\mathfrak{u}_{\lambda} \subset \mathfrak{u}$ is the weight subspace of weight $\lambda$ with respect to $S$. Let $\mathfrak{n}=\bigoplus_{\lambda \in \Phi} \mathfrak{n}_{\lambda}$ be the decomposition of the space $\mathfrak{n}$ into a direct sum of weight subspaces with respect to $S$; here, $\mathfrak{n}_{\lambda} \subset \mathfrak{u}_{\lambda}$ for all $\lambda \in \Phi$ and some of the subspaces $\mathfrak{n}_{\lambda}$ may be zero. For every $\lambda \in \Phi$ we denote by $c_{\lambda}$ the codimension of $\mathfrak{n}_{\lambda}$ in $\mathfrak{u}_{\lambda}$.

The following theorem gives a convenient sphericity criterion for connected solvable subgroups.

Theorem 1. Let $H \subset G$ be a connected solvable subgroup standardly embedded in $B$. Then the following conditions are equivalent:

(1) $H$ is spherical in $G$;

(2) $c_{\lambda} \leq 1$ for every $\lambda \in \Phi$, and all the weights $\lambda$ with $c_{\lambda}=1$ are linearly independent in $\mathfrak{X}(S)$.

Proof. By 3], Proposition I.1, 3), $H$ being spherical is equivalent to the condition that $S$ has an open orbit in $U / N$ under the action $(s, u N) \mapsto s u s^{-1} N$. By [11, Lemma 1.4, this condition is equivalent to the existence of an open orbit under the natural action of $S$ on $\mathfrak{u} / \mathfrak{n}$. It remains to prove that $S$ has an open orbit in $\mathfrak{u} / \mathfrak{n}$ if and only if condition (2) holds.

For each $\lambda \in \Phi$ with $c_{\lambda}>0$, we choose a subspace $\mathfrak{p}_{\lambda} \subset \mathfrak{u}_{\lambda}$ such that $\mathfrak{u}_{\lambda}=\mathfrak{n}_{\lambda} \oplus \mathfrak{p}_{\lambda}$. We set $\mathfrak{p}=\bigoplus_{\lambda \in \Phi: c_{\lambda}>0} \mathfrak{p}_{\lambda}$ so that $\mathfrak{u}=\mathfrak{n} \oplus \mathfrak{p}$. Then there is an $S$-equivariant isomorphism $\mathfrak{u} / \mathfrak{n} \simeq \mathfrak{p}$. We now show that condition (2) is equivalent to the existence of an open $S$-orbit in $\mathfrak{p}$. Indeed, suppose that condition (2) holds. We choose a nonzero element in each subspace $\mathfrak{p}_{\lambda}$. Then the set of elements we have selected is a basis in $\mathfrak{p}$. It is easy to see that an open $S$-orbit in $\mathfrak{p}$ is formed by those elements all of whose coordinates in the chosen basis are nonzero. Now suppose that condition (2) does not hold. We choose a basis in each subspace $\mathfrak{p}_{\lambda}$ and combine all these bases into a basis of $\mathfrak{p}$. If $c_{\lambda} \geq 2$ for some $\lambda \in \Phi$, then for any two different basis elements in $\mathfrak{p}_{\lambda}$ the ratio of the corresponding coordinate functions is a nonconstant $S$-invariant rational function on $\mathfrak{p}$, whence there is no open $S$-orbit in $\mathfrak{p}$. Now suppose that $c_{\lambda} \leq 1$ for all $\lambda \in \Phi$, but there are elements $\lambda_{1}, \ldots, \lambda_{k} \in \Phi$ such that $c_{\lambda_{1}}=\cdots=c_{\lambda_{k}}=1$ and $p_{1} \lambda_{1}+\cdots+p_{k} \lambda_{k}=0$ for some nonzero tuple $\left(p_{1}, \ldots, p_{k}\right) \in \mathbb{Z}^{k}$. Let $y_{1}, \ldots, y_{k}$ be the coordinate functions corresponding to the 
basis elements of the subspaces $\mathfrak{p}_{\lambda_{1}}, \ldots, \mathfrak{p}_{\lambda_{k}}$, respectively. Then it is easy to see that the nonconstant rational function $y_{1}^{p_{1}} \cdot \ldots \cdot y_{k}^{p_{k}}$ on $\mathfrak{p}$ is $S$-invariant; therefore there is no open $S$-orbit in $\mathfrak{p}$.

2.2. In this subsection we derive some consequences from Theorem 1 which will play a decisive role in our subsequent exposition.

First of all, recall the following well-known lemma from linear algebra.

Lemma 1. Suppose that vectors $v_{1}, \ldots, v_{n}$ of a finite-dimensional Euclidean space $V$ are contained in the same half-space and the angles between them are pairwise nonacute. Then these vectors are linearly independent.

Let $H \subset G$ be a connected solvable spherical subgroup standardly embedded in $B$. We set $S=H \cap T$ and $N=H \cap U$, so that $H=S \wedge N$. As $H$ is spherical, it satisfies condition (2) of Theorem 1. We denote all the weights $\lambda \in \Phi$ with $c_{\lambda}=1$ by $\varphi_{1}, \ldots, \varphi_{K}$. These weights are linearly independent in $\mathfrak{X}(S)$ and, in particular, each of them is nonzero. For $i=1, \ldots, K$ we denote by $\Psi_{i}$ the set of all roots $\alpha \in \Delta_{+}$such that $\tau(\alpha)=\varphi_{i}$ and $\mathfrak{g}_{\alpha} \not \subset \mathfrak{n}$. We set $\mathfrak{u}_{i}=\bigoplus_{\alpha \in \Psi_{i}} \mathfrak{g}_{\alpha}$. Clearly, $\mathfrak{u}_{i} \subset \mathfrak{u}_{\varphi_{i}}$ for all $i=1, \ldots, K$. Next, for $i=1, \ldots, K$ the subspace $\mathfrak{n} \cap \mathfrak{u}_{i} \subset \mathfrak{u}_{i}$ is the kernel of a linear function $\xi_{i} \in \mathfrak{u}_{i}^{*}$, which is determined up to proportionality. Clearly, if $\alpha \in \Psi_{i}$ for some $i \in\{1, \ldots, K\}$, then the restriction of $\xi_{i}$ to $\mathfrak{g}_{\alpha}$ is nonzero. We also set $\Psi=\Psi(H)=\Psi_{1} \cup \ldots \cup \Psi_{K}$. Note that $\Psi=\left\{\alpha \in \Delta_{+} \mid \mathfrak{g}_{\alpha} \not \subset \mathfrak{n}\right\}$.

Lemma 2. Suppose that $\alpha, \beta \in \Psi$ and $\gamma=\beta-\alpha \in \Delta_{+}$. Then $\gamma \notin \Psi$.

Proof. We have $\tau(\gamma)=\tau(\beta)-\tau(\alpha)$. If $\tau(\alpha)=\tau(\beta)$, then $\tau(\gamma)=0$, which is impossible for $\gamma \in \Psi$. If $\tau(\alpha) \neq \tau(\beta)$, then the weights $\tau(\alpha), \tau(\beta)$ are linearly independent and therefore both are distinct from $\tau(\gamma)$. We obtain that the weights $\tau(\alpha), \tau(\beta), \tau(\gamma)$ are pairwise distinct and linearly dependent, which is also impossible for $\gamma \in \Psi$.

Proposition 1. Suppose that $1 \leq i, j \leq K(i, j$ are not necessarily distinct $)$ and roots $\alpha \in \Psi_{i}, \beta \in \Psi_{j}$ are distinct. Suppose that $\gamma=\beta-\alpha \in \Delta_{+}$. Then $\Psi_{i}+\gamma \subset \Psi_{j}$. In particular, $\left|\Psi_{i}\right| \leq\left|\Psi_{j}\right|$.

Proof. It follows from Lemma 2 that $\gamma \notin \Psi$ and $\mathfrak{g}_{\gamma} \subset \mathfrak{n}$. Assume that $\alpha^{\prime}+\gamma \notin \Psi_{j}$ for some element $\alpha^{\prime} \in \Psi_{i}$. In the one-dimensional subspace $\left(\mathfrak{g}_{\alpha} \oplus \mathfrak{g}_{\alpha^{\prime}}\right) \cap \mathfrak{n}$, we choose a nonzero element $x=p e_{\alpha}+p^{\prime} e_{\alpha^{\prime}}$, where $p, p^{\prime} \in \mathbb{C}$. Note that $p \neq 0, p^{\prime} \neq 0$, and $\left[x, e_{\gamma}\right] \in \mathfrak{n}$. Let $q \neq 0$ be such that $\left[e_{\alpha}, e_{\gamma}\right]=q e_{\beta}$. Then $\left[x, e_{\gamma}\right]=p q e_{\beta}+p^{\prime}\left[e_{\alpha^{\prime}}, e_{\gamma}\right]$. If $\alpha^{\prime}+\gamma \in \Delta$, then the conditions $\tau\left(\alpha^{\prime}+\gamma\right)=\varphi_{j}$ and $\alpha^{\prime}+\gamma \notin \Psi_{j}$ imply $\left[e_{\alpha^{\prime}}, e_{\gamma}\right] \in \mathfrak{g}_{\alpha^{\prime}+\gamma} \subset \mathfrak{n}$, so that $e_{\beta} \in \mathfrak{n}$. If $\alpha^{\prime}+\gamma \notin \Delta$, then $\left[e_{\alpha^{\prime}}, e_{\gamma}\right]=0$, and again, $e_{\beta} \in \mathfrak{n}$. Thus, we have obtained that $\mathfrak{g}_{\beta} \subset \mathfrak{n}$, which contradicts the condition $\beta \in \Psi_{j}$.

Corollary 1. For each $j=1, \ldots, K$, the angles between the roots in $\Psi_{j}$ are pairwise nonacute, and these roots are linearly independent.

Proof. For $\left|\Psi_{j}\right|=1$ there is nothing to prove. For $\left|\Psi_{j}\right| \geq 2$, assume that two distinct roots $\alpha, \beta \in \Psi_{j}$ satisfy the condition $(\alpha, \beta)>0$. Then the vector $\gamma=\beta-\alpha$ is a root. We can assume without loss of generality that $\gamma \in \Delta_{+}$. Then by Proposition 1 we have $\Psi_{j}+\gamma \subset \Psi_{j}$, which is false. Therefore $(\alpha, \beta) \leq 0$ for any two distinct roots $\alpha, \beta \in \Psi_{j}$. The linear independence of the roots in $\Psi_{j}$ now follows from Lemma 1 .

Proposition 1 enables one to introduce a partial order on the set $\widetilde{\Psi}=\left\{\Psi_{1}, \ldots, \Psi_{K}\right\}$ as follows. For $i \neq j$ we write $\Psi_{i} \prec \Psi_{j}$ if $\Psi_{i}+\gamma \subset \Psi_{j}$ for some root $\gamma \in \Delta_{+}$. We write $\Psi_{i} \prec \Psi_{j}$ if $i=j$ or there exists a chain $\Psi_{i}=\Psi_{k_{1}}, \Psi_{k_{2}}, \ldots, \Psi_{k_{m-1}}, \Psi_{k_{m}}=\Psi_{j}$ such that $\Psi_{k_{p}} \prec \Psi_{k_{p+1}}$ for all $p=1, \ldots, m-1$. In particular, if $\Psi_{i} \prec \Psi_{j}$, then $\Psi_{i} \prec \Psi_{j}$. Clearly, the relation $\prec$ is transitive. Next, with each set $\Psi_{i}$ we associate the number 
$\rho\left(\Psi_{i}\right)=\sum_{\alpha \in \Psi_{i}}$ ht $\alpha$. Then, obviously, $\rho\left(\Psi_{i}\right)<\rho\left(\Psi_{j}\right)$ for $\Psi_{i} \prec \Psi_{j}$. Hence the relations $\Psi_{i} \prec \Psi_{j}$ and $\Psi_{j} \prec \Psi_{i}$ cannot hold simultaneously for $i \neq j$. Therefore, the relation $\prec$ is indeed a partial order on $\widetilde{\Psi}$.

For $i=1, \ldots, K$, we say that a root $\alpha \in \Psi_{i}$ is maximal if the set $\Psi_{i}$ is maximal in $\widetilde{\Psi}$ with respect to the partial order $\prec$.

Lemma 3. Let $\Psi_{i_{1}}, \ldots, \Psi_{i_{m}}$ be all the maximal elements of the set $\widetilde{\Psi}$ with respect to the order $\prec$. Then the angle between any pair of roots in the set $\Psi_{i_{1}} \cup \ldots \cup \Psi_{i_{m}}$ (that is, the maximal roots) is nonacute, and these roots are linearly independent.

Proof. In view of Lemma 1 and Corollary 1 it suffices to show that for $p \neq q$ the angle between any two roots $\alpha \in \Psi_{i_{p}}$ and $\beta \in \Psi_{i_{q}}$ is nonacute. Assume the converse. Then the difference $\gamma=\beta-\alpha$ is a root, and we can assume without loss of generality that $\gamma \in \Delta_{+}$. By Proposition 1 we obtain $\Psi_{i_{p}}+\gamma \subset \Psi_{i_{q}}$, whence $\Psi_{i_{p}} \prec \Psi_{i_{q}}$. This contradicts the maximality of the set $\Psi_{i_{p}}$ in $\widetilde{\Psi}$.

Proposition 2. Suppose that $1 \leq i, j \leq K, i \neq j, \Psi_{i} \prec \Psi_{j}$, and a root $\gamma \in \Delta_{+}$is such that $\Psi_{i}+\gamma \subset \Psi_{j}$. Then, up to proportionality, the linear function $\xi_{i} \in \mathfrak{u}_{i}^{*}$ is uniquely determined by the linear function $\xi_{j} \in \mathfrak{u}_{j}^{*}$. More precisely, there is a constant $c_{i j} \neq 0$ such that $\xi_{i}(x)=c_{i j} \xi_{j}\left(\left[x, e_{\gamma}\right]\right)$ for all $x \in \mathfrak{u}_{i}$.

Proof. Taking Lemma 2 into account, we obtain $\gamma \notin \Psi$, whence $\mathfrak{g}_{\gamma} \subset \mathfrak{n}$. It follows from the condition $\Psi_{i}+\gamma \subset \Psi_{j}$ that the linear map $l: \mathfrak{u}_{i} \rightarrow \mathfrak{u}_{j}, x \mapsto\left[x, e_{\gamma}\right]$, is injective. Consider the linear function $\xi_{i}^{\prime} \in \mathfrak{u}_{i}^{*}$ such that $\xi_{i}^{\prime}(x)=\xi_{j}(l(x))$ for $x \in \mathfrak{u}_{i}$. We have $\xi_{i}^{\prime} \not \equiv 0$, since $\xi_{j}\left(e_{\alpha}\right) \neq 0$ for any $\alpha \in \Psi_{j}$. As $l\left(\mathfrak{n} \cap \mathfrak{u}_{i}\right) \subset \mathfrak{n} \cap \mathfrak{u}_{j}$, it follows that $\xi_{i}^{\prime}(x)=0$ for any $x \in \mathfrak{n} \cap \mathfrak{u}_{i}$. This immediately implies that $\xi_{i}=c_{i j} \xi_{i}^{\prime}$ for some $c_{i j} \neq 0$, that is, $\xi_{i}(x)=c_{i j} \xi_{j}\left(\left[x, e_{\gamma}\right]\right)$ for all $x \in \mathfrak{u}_{i}$.

Theorem 2. Up to a conjugation by an element of $T$, a connected solvable spherical subgroup $H \subset G$ standardly embedded in $B$ is uniquely determined by its maximal torus $S \subset T$ and the set $\Psi \subset \Delta_{+}$.

Proof. The set of weights $\left\{\varphi_{1}, \ldots, \varphi_{K}\right\}$ is uniquely determined as the image of the set $\Psi$ under the map $\tau$. For every $i=1, \ldots, K$, the set $\Psi_{i}$ is uniquely determined as the set $\left\{\alpha \in \Psi \mid \tau(\alpha)=\varphi_{i}\right\}$. Next, by Proposition 2 the condition $\Psi_{i} \prec \Psi_{j}$ implies that, up to proportionality, the linear function $\xi_{i}$ is uniquely determined by the linear function $\xi_{j}$; therefore, up to proportionality, the whole family of linear functions $\xi_{1}, \ldots, \xi_{K}$ is uniquely determined by the linear functions $\xi_{j}$ corresponding to the maximal elements $\Psi_{j}$ of $\widetilde{\Psi}$.

The conjugation by an element $t \in T$ takes the algebra $\mathfrak{h}$ to an isomorphic one and acts on each of the spaces $\mathfrak{g}_{\alpha}, \alpha \in \Delta_{+}$, as the multiplication by $\alpha(t)$. By Lemma 3 all maximal roots of the set $\Psi$ are linearly independent. Therefore, for a suitable choice of $t \in T$, all the linear functions $\xi_{i}$ corresponding to the maximal elements $\Psi_{i}$ of $\widetilde{\Psi}$ can be independently reduced to a prescribed form. For example, we can require each $\xi_{i}$ to be the sum of all coordinates in the basis $\left\{e_{\alpha} \mid \alpha \in \Psi_{i}\right\}$. This is possible since $\left.\xi_{i}\right|_{\mathfrak{g}_{\alpha}} \not \equiv 0$ for any $\alpha \in \Psi_{i}$.

\section{§3. ACtive ROOT THEORY}

As we have seen in $\S 2.2$ (see Theorem 22), up to a conjugation by an element of $T$, a connected solvable spherical subgroup $H \subset G$ standardly embedded in $B$ is uniquely determined by its maximal torus $S \subset T$ and the set $\Psi \subset \Delta_{+}$. In this section we analyse the roots contained in $\Psi$ (these roots will be given the name "active" in $\S 3.1$ ) as well as the set $\Psi$ as a whole. 
Until the end of the section we fix a connected solvable spherical subgroup $H=$ $S \wedge N \subset G$ standardly embedded in $B$ (where $S=H \cap T$ and $N=H \cap U$ ) and retain all the notation introduced in $\S 2$.

3.1. In this subsection we introduce the notion of an active root, establish basic properties of active roots, and find out which positive roots can be active, depending on the root system $\Delta$.

Definition 1. A root $\alpha \in \Delta_{+}$is called active if $\mathfrak{g}_{\alpha} \not \subset \mathfrak{n}$.

Obviously, a root $\alpha$ is active if and only if $\alpha \in \Psi$.

Lemma 4. Let $\alpha$ be an active root and suppose that $\alpha=\beta+\gamma$, where $\beta, \gamma \in \Delta_{+}$. Then exactly one of the two roots $\beta, \gamma$ is active.

Proof. If neither of the roots $\beta, \gamma$ is active, then $\mathfrak{g}_{\beta}, \mathfrak{g}_{\gamma} \subset \mathfrak{n}$, whence $\mathfrak{g}_{\alpha}=\left[\mathfrak{g}_{\beta}, \mathfrak{g}_{\gamma}\right] \subset \mathfrak{n}$, which is not the case. Therefore at least one of the two roots $\beta, \gamma$ is active. By Lemma 2 these two roots cannot be active simultaneously.

Definition 2. We say that an active root $\beta$ is subordinate to an active root $\alpha$ if $\alpha=\beta+\gamma$ for some $\gamma \in \Delta_{+}$.

Definition 3. An active root $\alpha$ is called maximal if it is not subordinate to any other active root.

We note that the notion of maximality of an active root introduced in this definition coincides with the notion of maximality considered in $\$ 2.2$ In particular, if $\alpha$ is a maximal active root, then every active $\operatorname{root} \beta$ with $\tau(\alpha)=\tau(\beta)$ is also maximal.

Definition 4. If $\alpha$ is an active root, then the set consisting of $\alpha$ and all active roots subordinate to $\alpha$ is called the family of active roots generated by the active root $\alpha$. We denote this set by $F(\alpha)$.

For every root $\alpha \in \Delta_{+}$we set $s(\alpha)$ to be the number of representations of $\alpha$ as a sum of two positive roots. If a root $\alpha$ is active, then by Lemma 4 the number of active roots subordinate to $\alpha$ is equal to $s(\alpha)$, that is, $s(\alpha)=|F(\alpha)|-1$.

Lemma 5. Let $\alpha$ be an active root.

(a) If $\beta \in F(\alpha) \backslash\{\alpha\}$, then $\tau(\alpha) \neq \tau(\beta)$.

(b) If $\beta, \gamma \in F(\alpha) \backslash\{\alpha\}$ and $\beta \neq \gamma$, then $\tau(\beta) \neq \tau(\gamma)$.

Proof. (a) We set $\gamma=\alpha-\beta \in \Delta_{+}$. Assume that $\tau(\alpha)=\tau(\beta)$. Then $\alpha, \beta \in \Psi_{i}$ for some $i \in\{1, \ldots, K\}$. By Proposition 1 we have $\Psi_{i}+\gamma \subset \Psi_{i}$, which is not the case. Therefore, $\tau(\alpha) \neq \tau(\beta)$.

(b) Suppose that $\alpha=\beta+\beta^{\prime}=\gamma+\gamma^{\prime}$, where $\beta^{\prime}, \gamma^{\prime} \in \Delta_{+}$and $\beta^{\prime} \neq \gamma^{\prime}$. Fix $i \in\{1, \ldots, K\}$ such that $\alpha \in \Psi_{i}$. Assume that $\tau(\beta)=\tau(\gamma)$. Then $\tau\left(\beta^{\prime}\right)=\tau\left(\gamma^{\prime}\right)$. Next, by Proposition 1 we obtain that $\beta+\gamma^{\prime}, \gamma+\beta^{\prime} \in \Psi_{i}$, whence $\tau\left(\beta+\gamma^{\prime}\right)=\tau\left(\gamma+\beta^{\prime}\right)=\tau(\alpha)$. Note that the roots $\alpha, \beta+\gamma^{\prime}, \gamma+\beta^{\prime}$ are distinct because $\beta \neq \gamma$. By Corollary 1 these three roots are linearly independent. On the other hand, they satisfy the linear dependence $2 \alpha=\left(\beta+\gamma^{\prime}\right)+\left(\gamma+\beta^{\prime}\right)$, a contradiction. Therefore, $\tau(\beta) \neq \tau(\gamma)$.

Corollary 2. If $\alpha$ is an active root, then all roots in $F(\alpha)$ are linearly independent.

Proof. It follows from Lemma 5 that all weights $\tau(\beta)$, where $\beta \in F(\alpha)$, are distinct. By Theorem 1 these weights are linearly independent. Therefore, a fortiori, all roots in $F(\alpha)$ are linearly independent. 
Lemma 6. Suppose that $\alpha \in \Delta_{+}$.

(a) If $\Delta(\alpha)$ is a root system of type $\mathrm{A}, \mathrm{D}$, or $\mathrm{E}$, then $s(\alpha)=$ ht $\alpha-1$.

(b) In the general case, $s(\alpha) \geq|\operatorname{Supp} \alpha|-1$.

Proof. We assume without loss of generality that $\Delta=\Delta(\alpha)$.

Let us first prove (a). Since the root system $\Delta$ has type A, D, or E, the lengths of all roots in it are the same. Therefore,

(1) the sum of two roots is a root if and only if the angle between them is $2 \pi / 3$;

(2) the difference of two roots is a root if and only if the angle between them is $\pi / 3$;

(3) for any $\beta \in \Delta$ and $\beta_{0} \in \Pi$ the root $r_{\beta_{0}}(\beta)$ is equal either to $\beta-\beta_{0}$, or to $\beta$, or to $\beta+\beta_{0}$.

We use induction on ht $\alpha$. For ht $\alpha=1$ the assertion is true. Assume that ht $\alpha=k$ and the assertion has been established for all roots $\alpha^{\prime} \in \Delta_{+}$with ht $\alpha^{\prime}<k$. Consider an arbitrary simple root $\alpha_{0}$ such that $\beta=\alpha-\alpha_{0} \in \Delta_{+}$. Then the angle between the roots $\alpha_{0}$ and $\beta$ is equal to $2 \pi / 3$, whence $\alpha=r_{\alpha_{0}}(\beta)$. We have ht $\beta=$ ht $\alpha-1$; therefore $s(\beta)=$ ht $\alpha-2$ by the induction hypothesis. Suppose that $\beta=\beta_{1}+\beta_{2}$, where $\beta_{1}, \beta_{2} \in \Delta_{+}$. Note that neither of the sets $\operatorname{Supp} \beta_{1}$, Supp $\beta_{2}$ coincides with $\left\{\alpha_{i}\right\}$, because otherwise one of the roots $\beta_{1}, \beta_{2}$ would coincide with $\alpha_{i}$, which is impossible, since $\beta-\alpha_{i}$ is not a root. Consequently, $r_{\alpha_{0}}\left(\beta_{1}\right), r_{\alpha_{0}}\left(\beta_{2}\right) \in \Delta_{+} \backslash\left\{\alpha_{0}\right\}$, and $\alpha=r_{\alpha_{0}}\left(\beta_{1}\right)+r_{\alpha_{0}}\left(\beta_{2}\right)$ is a representation of $\alpha$ as a sum of two positive roots. Conversely, if $\alpha=\alpha_{1}+\alpha_{2}$, where $\alpha_{1}, \alpha_{2} \in \Delta_{+} \backslash\left\{\alpha_{0}\right\}$, then Supp $\alpha_{1} \neq\left\{\alpha_{0}\right\}$ and Supp $\alpha_{2} \neq\left\{\alpha_{0}\right\}$, so $r_{\alpha_{0}}\left(\alpha_{1}\right), r_{\alpha_{0}}\left(\alpha_{2}\right) \in \Delta_{+}$ and $\beta=r_{\alpha_{0}}\left(\alpha_{1}\right)+r_{\alpha_{0}}\left(\alpha_{2}\right)$ is a representation of $\beta$ as a sum of two positive roots. Thus, we have established a one-to-one correspondence between the representations of $\beta$ as a sum of two positive roots and the representations of $\alpha$ as a sum of two positive roots different from $\alpha_{0}$. Taking the representation $\alpha=\alpha_{0}+\beta$ into account, we obtain $s(\alpha)=s(\beta)+1=$ ht $\alpha-1$.

We now prove (b). We again use induction on ht $\alpha$. For ht $\alpha=1$ the assertion holds. Assume that ht $\alpha=k$ and the assertion holds for all roots $\alpha^{\prime} \in \Delta_{+}$with ht $\alpha^{\prime}<k$. In view of Lemma 1 there exists a simple root $\alpha_{0}$ such that $\left(\alpha, \alpha_{0}\right)>0$. Then $\beta=\alpha-\alpha_{0} \in \Delta_{+}$. We set $\gamma=r_{\alpha_{0}}(\alpha)$. Since $\left(\alpha, \alpha_{0}\right)>0$, we have ht $\gamma<$ ht $\alpha$; therefore the root $\gamma$ satisfies the induction hypothesis. Namely, $s(\gamma) \geq|\operatorname{Supp} \alpha|-2$ for $\alpha_{0} \notin \operatorname{Supp} \gamma$ and $s(\gamma) \geq|\operatorname{Supp} \alpha|-1$ for $\alpha_{0} \in \operatorname{Supp} \gamma$. In any case, the number of representations of the form $\gamma=\gamma_{1}+\gamma_{2}$, where $\gamma_{1}, \gamma_{2} \in \Delta_{+} \backslash\left\{\alpha_{0}\right\}$, is at least $|\operatorname{Supp} \alpha|-2$. For every such representation, we have $r_{\alpha_{0}}\left(\gamma_{1}\right), r_{\alpha_{0}}\left(\gamma_{2}\right) \in \Delta_{+} \backslash\left\{\alpha_{0}\right\}$; therefore $\alpha=r_{\alpha_{0}}\left(\gamma_{1}\right)+r_{\alpha_{0}}\left(\gamma_{2}\right)$ is a representation of the root $\alpha$ as a sum of two positive roots. Taking account of the representation $\alpha=\beta+\alpha_{0}$, we obtain $s(\alpha) \geq|\operatorname{Supp} \alpha|-1$.

Lemma 7. Let $\alpha$ be an active root. Then:

(a) $|F(\alpha)|=|\operatorname{Supp} \alpha|$;

(b) all weights $\tau(\beta)$, where $\beta \in \operatorname{Supp} \alpha$, are linearly independent;

(c) $\langle F(\alpha)\rangle=\langle\operatorname{Supp} \alpha\rangle$;

(d) if $\beta \in \Delta_{+}, \operatorname{Supp} \beta \subset \operatorname{Supp} \alpha$, and $\alpha-\beta \notin \Delta_{+}$, then the root $\beta$ is not active.

Proof. By Lemma 6(b) we have $|F(\alpha)| \geq|\operatorname{Supp} \alpha|$. By Lemma [5 all weights $\tau(\gamma)$, where $\gamma \in F(\alpha)$, are distinct, and they are linearly independent by Theorem 1 . But these weights are contained in the subspace $\tau(\langle\operatorname{Supp} \alpha\rangle) \subset \mathfrak{X}(S) \otimes_{\mathbb{Z}} \mathbb{Q}$ of dimension at most $|\operatorname{Supp} \alpha|$; therefore, $|F(\alpha)| \leq|\operatorname{Supp} \alpha|$. This proves parts (a), (b), and (c).

We now prove (d). Suppose that $\beta \in \Delta_{+}$, Supp $\beta \subset \operatorname{Supp} \alpha$, and $\alpha-\beta \notin \Delta_{+}$. Then from (b) we see that $\tau(\beta) \neq \tau(\gamma)$ for all $\gamma \in F(\alpha)$. If the root $\beta$ were active, then by Theorem 1 all weights in the set $\{\tau(\beta)\} \cup\{\tau(\gamma) \mid \gamma \in F(\alpha)\}$ would be linearly independent, which is impossible by (c). Therefore $\beta$ is not active. 
Corollary 3. Let $\alpha$ be an active root.

(a) If $\beta \in \Psi$ and $\operatorname{Supp} \beta \subset \operatorname{Supp} \alpha$, then $\beta \in F(\alpha)$.

(b) If $\beta \in F(\alpha) \backslash\{\alpha\}$, then $F(\beta) \subset F(\alpha)$.

(c) If $\alpha$ is maximal, then $\operatorname{Supp} \alpha \backslash \operatorname{Supp} \beta \neq \varnothing$ for any maximal active root $\beta \neq \alpha$.

Proof. Under the hypotheses of part (a), by Lemma 7(d) we obtain $\alpha-\beta \in \Delta_{+}$, whence $\beta \in F(\alpha)$. Parts (b) and (c) obviously follow from (a).

Corollary 4. Suppose that $\Psi_{i} \prec \Psi_{j}$ for some $i, j \in\{1, \ldots, K\}, i \neq j$. Then $\Psi_{i} \prec \Psi_{j}$.

Proof. It suffices to prove that $\Psi_{p} \prec \Psi_{r}$ for any $p, q, r$ which satisfy $\Psi_{p} \prec \Psi_{q}, \Psi_{q} \prec \Psi_{r}$. By the definition of the partial order on $\widetilde{\Psi}$, there exist roots $\gamma_{p q}, \gamma_{q r} \in \Delta_{+}$such that $\Psi_{p}+\gamma_{p q} \subset \Psi_{q}$ and $\Psi_{q}+\gamma_{q r} \subset \Psi_{r}$. Consider an arbitrary root $\alpha \in \Psi_{p}$. Then $\alpha+\gamma_{p q} \in \Psi$, $\alpha+\gamma_{p q}+\gamma_{q r} \in \Psi, \alpha \in F\left(\alpha+\gamma_{p q}\right)$, and $\alpha+\gamma_{p q} \in F\left(\alpha+\gamma_{p q}+\gamma_{q r}\right)$. By Corollary 3 we obtain that $\alpha \in F\left(\alpha+\gamma_{p q}+\gamma_{q r}\right)$. Therefore, $\gamma_{p q}+\gamma_{q r} \in \Delta_{+}$, so by Proposition 1 we have $\Psi_{p}+\left(\gamma_{p q}+\gamma_{q r}\right) \subset \Psi_{r}$, that is, $\Psi_{p} \prec \Psi_{r}$.

Proposition 3. Let $\alpha$ be an active root. Then there is a unique simple root $\pi(\alpha) \in$ Supp $\alpha$ with the following property: if $\alpha=\alpha_{1}+\alpha_{2}$ for some roots $\alpha_{1}, \alpha_{2} \in \Delta_{+}$, then $\alpha_{1}$ (resp. $\left.\alpha_{2}\right)$ is active if and only if $\pi(\alpha) \notin \operatorname{Supp} \alpha_{1}$ (resp. $\left.\pi(\alpha) \notin \operatorname{Supp} \alpha_{2}\right)$.

Proof. We prove this assertion by induction on ht $\alpha$. If ht $\alpha=1$, then $\alpha \in \Pi$ and we can take $\pi(\alpha)=\alpha$. Now suppose that ht $\alpha=k$ and that the assertion holds for all active roots of height at most $k-1$. Assume that the required root $\pi(\alpha)$ does not exist. With every simple root $\gamma \in \operatorname{Supp} \alpha$ we associate an active root $\gamma^{\prime} \in F(\alpha) \backslash\{\alpha\}$ such that $\gamma \in \operatorname{Supp} \gamma^{\prime}$ and the height of the root $\gamma^{\prime}$ is minimal. Then $\gamma=\pi\left(\gamma^{\prime}\right)$ in view of the choice of $\gamma^{\prime}$ and the induction hypothesis. From the uniqueness of the root $\pi\left(\gamma^{\prime}\right)$ we obtain that for distinct roots $\gamma_{1}, \gamma_{2} \in \operatorname{Supp} \alpha$ the corresponding roots $\gamma_{1}^{\prime}, \gamma_{2}^{\prime} \in F(\alpha)$ are also distinct. Thus, $|F(\alpha)| \geq|\operatorname{Supp} \alpha|+1$, which contradicts Lemma 7(a). Therefore a root $\pi(\alpha)$ with the requisite properties does exist. If there is another such simple root $\pi^{\prime}(\alpha) \neq \pi(\alpha)$, then the set $F(\alpha) \backslash\{\alpha\}$ has $|\operatorname{Supp} \alpha|-1$ elements, is linearly independent by Corollary 2 , and is contained in the subspace $\left\langle(\operatorname{Supp} \alpha) \backslash\left\{\pi(\alpha), \pi^{\prime}(\alpha)\right\}\right\rangle$ of dimension $|\operatorname{Supp} \alpha|-2$, which is impossible. Thus, the root $\pi(\alpha)$ is uniquely determined.

Corollary 5. For every active root $\alpha$, the family of active roots $F(\alpha)$ is uniquely determined by the simple root $\pi(\alpha)$.

Corollary 6. If $\alpha$ is an active root, then the map $\pi: F(\alpha) \rightarrow \operatorname{Supp} \alpha$ is a bijection.

Proof. With each simple root $\beta \in \operatorname{Supp} \alpha$ we associate a root $\rho(\beta) \in F(\alpha)$ of minimal height such that $\beta \in \operatorname{Supp} \rho(\beta)$ (if there are several, we choose any one). Then by Proposition 3 applied to the active root $\rho(\beta)$ we obtain $\beta=\pi(\rho(\beta))$; therefore the map $\pi$ is surjective. But $|F(\alpha)|=|\operatorname{Supp} \alpha|$ by Lemma $7($ a), whence $\pi$ is a bijection.

Definition 5. If $\alpha$ is an active root, then the root $\pi(\alpha) \in \Pi$ appearing in Proposition 3 is called the simple root associated with the active root $\alpha$.

Theorem 3. Suppose that $\alpha$ is an active root and $\pi(\alpha)$ is the simple root associated with $\alpha$. Then the pair $(\alpha, \pi(\alpha))$ is contained in Table 1 .

This theorem being proved a bit later, we now explain the notation in Table1 Column " $\alpha$ " gives the expression of $\alpha$ as a sum of simple roots in Supp $\alpha$. Here, the $j$ th simple root in the diagram $\Sigma(\operatorname{Supp} \alpha)$ is denoted by $\alpha_{j}$. Column " $\pi(\alpha)$ " lists all possibilities for $\pi(\alpha)$ for a given active root $\alpha$. 
TABLE 1

\begin{tabular}{|c|c|c|c|}
\hline & Type of $\Delta(\alpha)$ & $\alpha$ & $\pi(\alpha)$ \\
\hline 1 & any of rank $n$ & $\alpha_{1}+\alpha_{2}+\cdots+\alpha_{n}$ & $\alpha_{1}, \alpha_{2}, \ldots, \alpha_{n}$ \\
\hline 2 & $\mathrm{~B}_{n}$ & $\alpha_{1}+\alpha_{2}+\cdots+\alpha_{n-1}+2 \alpha_{n}$ & $\alpha_{1}, \alpha_{2}, \ldots, \alpha_{n-1}$ \\
\hline 3 & $\mathrm{C}_{n}$ & $2 \alpha_{1}+2 \alpha_{2}+\cdots+2 \alpha_{n-1}+\alpha_{n}$ & $\alpha_{n}$ \\
\hline 4 & $\mathrm{~F}_{4}$ & $2 \alpha_{1}+2 \alpha_{2}+\alpha_{3}+\alpha_{4}$ & $\alpha_{3}, \alpha_{4}$ \\
\hline 5 & $\mathrm{G}_{2}$ & $2 \alpha_{1}+\alpha_{2}$ & $\alpha_{2}$ \\
\hline 6 & $\mathrm{G}_{2}$ & $3 \alpha_{1}+\alpha_{2}$ & $\alpha_{2}$ \\
\hline
\end{tabular}

Proof of Theorem 3. If $\Delta(\alpha)$ is a root system of type A, D, or E, then by Lemmas 6 and 7 the root $\alpha$ is equal to the sum of simple roots in its support.

If, however, $\Delta(\alpha)$ has one of the types B, C, F, G, then by Lemma 7(a) we obtain $s(\alpha)=|\operatorname{Supp}(\alpha)|-1$. By a case-by-case analysis it is easy to establish that this equality holds for exactly two roots with complete support 11 in the root systems of types $\mathrm{B}_{n}, \mathrm{C}_{n}, \mathrm{~F}_{4}$, and for exactly three roots with complete support in the root system $G_{2}$. All these roots are contained in Table 1. For each of the rows $2-5$ of this table, consider the $\operatorname{root} \beta=\sum_{\gamma \in \operatorname{Supp} \alpha} \gamma \in \Delta_{+}$. We have $\alpha-\beta \in \Delta_{+}$; therefore $\alpha=\beta+(\alpha-\beta)$ is a representation of $\alpha$ as a sum of two positive roots. Hence, $\pi(\alpha) \notin \operatorname{Supp}(\alpha-\beta)$. For the root $\alpha$ in row 6 of Table 1, $\alpha=\alpha_{1}+\left(2 \alpha_{1}+\alpha_{2}\right)$ is a representation of $\alpha$ as a sum of two positive roots, so $\pi(\alpha) \neq \alpha_{1}$. Thus, for every root $\alpha$ in rows $2-6$ of Table 1 , we have obtained a subset of the set $\operatorname{Supp} \alpha$ that certainly does not contain the root $\pi(\alpha)$. All the remaining possibilities for $\pi(\alpha)$ are listed in each case in column " $\pi(\alpha)$ ".

Remark 1 . From the existence theorem proved in $\S 4$ below it follows that all the possibilities presented in Table 1 are realized.

In order to state some corollaries of Theorem 3, we introduce the following concept.

Definition 6. Let $\alpha$ be an active root. A simple root $\alpha^{\prime} \in \operatorname{Supp} \alpha$ is said to be terminal with respect to $\operatorname{Supp} \alpha$ if in the diagram $\Sigma(\operatorname{Supp} \alpha)$ the node $\alpha^{\prime}$ is connected by an edge to exactly one node.

The following three assertions are obtained by an easy case-by-case examination of all the possibilities in Table 1 .

Corollary 7. If $\alpha$ is an active root, $|\operatorname{Supp} \alpha| \geq 2$, and $\alpha^{\prime} \in \operatorname{Supp} \alpha \cap F(\alpha)$, then the root $\alpha^{\prime}$ is terminal with respect to $\operatorname{Supp} \alpha$.

Corollary 8. If $\alpha$ is an active root and a simple root $\alpha^{\prime} \in \operatorname{Supp} \alpha$ is terminal with respect to $\operatorname{Supp} \alpha$, then either $\alpha^{\prime}=\pi(\alpha)$ or $\alpha^{\prime} \in F(\alpha)$.

Corollary 9. Suppose that $\alpha, \alpha^{\prime}$ are active roots such that $\alpha^{\prime} \in F(\alpha), \pi(\alpha)$ is terminal with respect to $\operatorname{Supp} \alpha$, and in the diagram $\Sigma(\operatorname{Supp} \alpha)$ the node $\pi\left(\alpha^{\prime}\right)$ is connected by an edge to the node $\pi(\alpha)$. Then $\operatorname{Supp} \alpha=\{\pi(\alpha)\} \cup \operatorname{Supp} \alpha^{\prime}$.

3.2. In this subsection we find out how the supports of two different maximal active roots can intersect. Our main results here are Propositions 4 and 5.

Lemma 8. Let $\alpha, \beta$ be distinct maximal active roots such that $\tau(\alpha) \neq \tau(\beta)$. Suppose that $\alpha^{\prime} \in F(\alpha), \beta^{\prime} \in F(\beta)$, and $\alpha^{\prime} \neq \beta^{\prime}$. Then $\tau\left(\alpha^{\prime}\right) \neq \tau\left(\beta^{\prime}\right)$.

\footnotetext{
${ }^{1}$ For $\Delta$ indecomposable, a root $\alpha \in \Delta_{+}$has complete support (with respect to $\Pi$ ) if $\operatorname{Supp} \alpha=\Pi$.
} 
Proof. If $\alpha^{\prime}=\alpha$ and $\beta^{\prime}=\beta$, then there is nothing to prove. Therefore we assume without loss of generality that $\alpha^{\prime} \neq \alpha$ and $\alpha=\alpha^{\prime}+\alpha^{\prime \prime}$ for some $\alpha^{\prime \prime} \in \Delta_{+}$. Assume that $\tau\left(\alpha^{\prime}\right)=\tau\left(\beta^{\prime}\right)$. If $\beta^{\prime}=\beta$, then Proposition 1 implies that $\beta^{\prime}+\alpha^{\prime \prime}=\beta+\alpha^{\prime \prime}$ is an active root, which contradicts the maximality of $\beta$. Therefore in what follows we assume that $\beta^{\prime} \neq \beta$ and $\beta=\beta^{\prime}+\beta^{\prime \prime}$ for some $\beta^{\prime \prime} \in \Delta_{+}$. Again by Proposition 1 we obtain that $\alpha^{\prime}+\beta^{\prime \prime}$ and $\beta^{\prime}+\alpha^{\prime \prime}$ are active roots such that $\tau\left(\alpha^{\prime}+\beta^{\prime \prime}\right)=\tau(\beta)$ and $\tau\left(\beta^{\prime}+\alpha^{\prime \prime}\right)=\tau(\alpha)$. Then by Lemma 3 the angles between the (distinct) roots $\alpha, \beta^{\prime}+\alpha^{\prime \prime}, \beta, \alpha^{\prime}+\beta^{\prime \prime}$ are nonacute, and therefore these roots are linearly independent. On the other hand, there is the linear dependence $\alpha+\beta=\left(\beta^{\prime}+\alpha^{\prime \prime}\right)+\left(\alpha^{\prime}+\beta^{\prime \prime}\right)$. This contradiction proves that $\tau\left(\alpha^{\prime}\right) \neq \tau\left(\beta^{\prime}\right)$.

Lemma 9. Suppose that $\alpha, \beta$ are distinct maximal active roots such that $\tau(\alpha) \neq \tau(\beta)$. Then neither of the simple roots $\pi(\alpha), \pi(\beta)$ is contained in the set $\operatorname{Supp} \alpha \cap \operatorname{Supp} \beta$.

Proof. It suffices to prove that $\pi(\alpha) \notin \operatorname{Supp} \alpha \cap \operatorname{Supp} \beta$. Assume the converse. We set $a=|\operatorname{Supp} \alpha|, b=|\operatorname{Supp} \beta|$, and $c=|\operatorname{Supp} \alpha \cap \operatorname{Supp} \beta|$. By Lemma 6, the set $\{\gamma \in F(\alpha) \mid \pi(\gamma) \in \operatorname{Supp} \alpha \backslash \operatorname{Supp} \beta\}$ contains at least $a-c$ roots. Clearly, none of these roots, nor the root $\alpha$, is contained in the set $F(\beta)$. Therefore, there are at least $a-c+1+b$ distinct roots in the set $F(\alpha) \cup F(\beta)$. By Lemmas [5] and 8 we obtain that the weights with respect to $S$ of all roots in $F(\alpha) \cup F(\beta)$ are different and, by Theorem 1, linearly independent. Hence the dimension of the space $\langle F(\alpha) \cup F(\beta)\rangle$ is at least $a+b-c+1$. On the other hand, this space is contained in the space $\langle\operatorname{Supp} \alpha \cup \operatorname{Supp} \beta\rangle$ of dimension $a+b-c$, and we obtain a contradiction.

Corollary 10. Suppose that $\alpha$ and $\beta$ are distinct maximal active roots and $\pi(\alpha) \in$ $\operatorname{Supp} \alpha \cap \operatorname{Supp} \beta$. Then $\tau(\alpha)=\tau(\beta)$.

Next we list some conditions on a pair of active roots $\alpha, \beta$. These conditions will be used when we state Propositions 4 and 5.

(D0) $\operatorname{Supp} \alpha \cap \operatorname{Supp} \beta=\varnothing$;

(D1) Supp $\alpha \cap \operatorname{Supp} \beta=\{\delta\}$, where $\pi(\alpha) \neq \delta, \pi(\beta) \neq \delta$, and the root $\delta$ is terminal with respect to $\operatorname{Supp} \alpha$ and $\operatorname{Supp} \beta$;

(E1) Supp $\alpha \cap \operatorname{Supp} \beta=\{\delta\}$, where $\delta=\pi(\alpha)=\pi(\beta), \alpha-\delta \in \Delta_{+}, \beta-\delta \in \Delta_{+}$, and the root $\delta$ is terminal with respect to $\operatorname{Supp} \alpha$ and $\operatorname{Supp} \beta$;

(D2) the diagram $\Sigma(\operatorname{Supp} \alpha \cup \operatorname{Supp} \beta)$ has the form depicted in Figure1 (where $p, q, r \geq$ 1 are some numbers), $\alpha=\alpha_{1}+\cdots+\alpha_{p}+\gamma_{0}+\gamma_{1}+\cdots+\gamma_{r}, \beta=\beta_{1}+\cdots+\beta_{q}+\gamma_{0}+\gamma_{1}+\cdots+\gamma_{r}$, $\pi(\alpha) \notin \operatorname{Supp} \alpha \cap \operatorname{Supp} \beta$, and $\pi(\beta) \notin \operatorname{Supp} \alpha \cap \operatorname{Supp} \beta$;

(E2) the diagram $\Sigma(\operatorname{Supp} \alpha \cup \operatorname{Supp} \beta$ ) has the form depicted in Figure1(where $p, q, r \geq$ 1 are some numbers), $\alpha=\alpha_{1}+\cdots+\alpha_{p}+\gamma_{0}+\gamma_{1}+\cdots+\gamma_{r}, \beta=\beta_{1}+\cdots+\beta_{q}+\gamma_{0}+\gamma_{1}+\cdots+\gamma_{r}$, and $\pi(\alpha)=\pi(\beta) \in \operatorname{Supp} \alpha \cap \operatorname{Supp} \beta$.

We note that in condition (D1) the root $\delta$ is active by Corollary 8 .

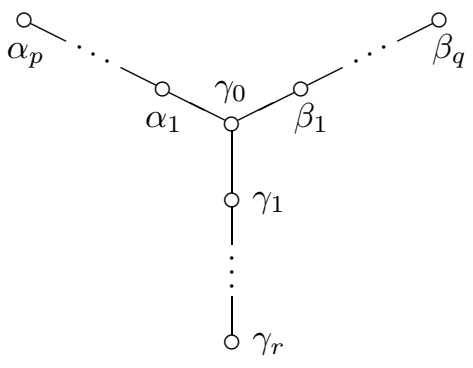

Figure 1 
Proposition 4. Suppose that $\alpha, \beta$ are distinct maximal active roots such that $\tau(\alpha) \neq$ $\tau(\beta)$. Then exactly one of the possibilities (D0), (D1) or (D2) is realized.

Proof. We can assume without loss of generality that $\Delta=\Delta \cap\langle\operatorname{Supp} \alpha \cup \operatorname{Supp} \beta\rangle$. Set $I=\operatorname{Supp} \alpha \cap \operatorname{Supp} \beta$. Assume that possibility (D0) is not realized and $I \neq \varnothing$. Then in the diagram $\Sigma(\Pi)$ there exists a node $\delta \in I$ connected by an edge to a node contained in Supp $\alpha \backslash I$ (the latter set is nonempty in view of the maximality of $\alpha$ and Corollary 3(a)). Two cases are possible.

Case 1. The root $\delta$ is terminal with respect to $\operatorname{Supp} \alpha$ or $\operatorname{Supp} \beta$. Then, by Corollary 8 and Lemma 9, $\delta$ is active. In view of Corollary 7 we obtain that $\delta$ is terminal with respect to both $\operatorname{Supp} \alpha$ and $\operatorname{Supp} \beta$; therefore, $I=\{\delta\}$ and condition (D1) holds.

Case 2. The root $\delta$ is terminal with respect to neither $\operatorname{Supp} \alpha$ nor $\operatorname{Supp} \beta$. Since the situation is symmetric with respect to interchanging $\alpha$ and $\beta$, we can assume that the following additional condition holds: every node in $I$ connected by an edge to a node in $(\operatorname{Supp} \alpha \cup \operatorname{Supp} \beta) \backslash I$ is terminal with respect to neither $\operatorname{Supp} \alpha$ nor $\operatorname{Supp} \beta$. It follows from this condition that in the diagram $\Sigma(\Pi)$ the node $\delta$ has degree 3 , and the diagram itself has the form depicted in Figure1 (for some $p, q, r \geq 1$ ), and $\alpha=\alpha_{1}+\cdots+\alpha_{p}+\gamma_{0}+\gamma_{1}+\cdots+\gamma_{r}$, $\beta=\beta_{1}+\cdots+\beta_{q}+\gamma_{0}+\gamma_{1}+\cdots+\gamma_{r}, \delta=\gamma_{0}$. By Lemma 9 neither of the simple roots $\pi(\alpha), \pi(\beta)$ is contained in $I$; therefore condition (D2) holds.

Lemma 10. Let $\alpha, \beta$ be distinct active roots such that $\pi(\alpha)=\pi(\beta)$. Then $\tau(\alpha)=\tau(\beta)$.

Proof. By Corollary [6] we have $\alpha \notin F(\beta)$ and $\beta \notin F(\alpha)$. We set $\delta=\pi(\alpha)=\pi(\beta)$, $a=|\operatorname{Supp} \alpha|, b=|\operatorname{Supp} \beta|$, and $c=|\operatorname{Supp} \alpha \cap \operatorname{Supp} \beta|$. Assume that $\tau(\alpha) \neq \tau(\beta)$. Consider the set $A=F(\alpha) \cup\{\beta\} \cup\{\gamma \in F(\beta) \mid \pi(\gamma) \in \operatorname{Supp} \beta \backslash \operatorname{Supp} \alpha\}$. This set consists of exactly $a+b-c+1$ different elements. If $\operatorname{dim} \tau(\langle\operatorname{Supp} \alpha \cup \operatorname{Supp} \beta\rangle)=a+b-c$, then the weights with respect to $S$ of all elements in $A$ are different and therefore linearly independent (Theorem 11). But this is impossible, since $A \subset\langle\operatorname{Supp} \alpha \cup \operatorname{Supp} \beta\rangle$. Therefore, $\operatorname{dim} \tau(\langle\operatorname{Supp} \alpha \cup \operatorname{Supp} \beta\rangle) \leq a+b-c-1$ and so we can find at least two pairs of elements in $A$ with the same weights with respect to $S$. Two cases are possible.

Case 1. There are roots $\alpha^{\prime} \in F(\alpha) \backslash\{\alpha\}$ and $\beta^{\prime} \in A \cap F(\beta) \backslash\{\beta\}$ such that $\tau(\alpha)=\tau\left(\beta^{\prime}\right)$ and $\tau(\beta)=\tau\left(\alpha^{\prime}\right)$. Let $i, j \in\{1, \ldots, K\}$ be such that $\alpha \in \Psi_{i}$ and $\beta \in \Psi_{j}$. Then we have $\Psi_{i} \prec \Psi_{j}$ and $\Psi_{j} \prec \Psi_{i}$, so $\Psi_{i}=\Psi_{j}$ and $\tau(\alpha)=\tau(\beta)$, a contradiction.

Case 2. There are roots $\alpha^{\prime} \in F(\alpha) \backslash\{\alpha\}$ and $\beta^{\prime} \in A \cap F(\beta) \backslash\{\beta\}$ such that $\tau\left(\alpha^{\prime}\right)=$ $\tau\left(\beta^{\prime}\right)$. Note that $\alpha^{\prime} \neq \beta^{\prime}$. Let $\operatorname{roots} \alpha^{\prime \prime}, \beta^{\prime \prime} \in \Delta_{+}$be such that $\alpha=\alpha^{\prime}+\alpha^{\prime \prime}$ and $\beta=\beta^{\prime}+\beta^{\prime \prime}$. Then by Proposition 1 we have $\alpha^{\prime}+\beta^{\prime \prime}, \beta^{\prime}+\alpha^{\prime \prime} \in \Psi$ and $\tau\left(\alpha^{\prime}+\beta^{\prime \prime}\right)=\tau(\beta)$, $\tau\left(\beta^{\prime}+\alpha^{\prime \prime}\right)=\tau(\alpha)$. Since $\alpha^{\prime} \neq \beta^{\prime}$ and $\tau(\alpha) \neq \tau(\beta)$, the four roots $\alpha, \beta, \alpha^{\prime}+\beta^{\prime \prime}, \beta^{\prime}+\alpha^{\prime \prime}$ are pairwise distinct. By Corollary 1 the angle between $\alpha^{\prime}+\beta^{\prime \prime}$ and $\beta$, as well as the angle between $\beta^{\prime}+\alpha^{\prime \prime}$ and $\alpha$, is nonacute. Next, the angle between the $\operatorname{roots} \alpha$ and $\beta$ is also nonacute, since otherwise their difference would be a root and we would have $\alpha \in F(\beta)$ or $\beta \in F(\alpha)$, so that $\pi(\alpha) \neq \pi(\beta)$ (Corollary 6), which is not the case. Assume that the angle between either $\alpha^{\prime}+\beta^{\prime \prime}$ and $\alpha$ or $\beta^{\prime}+\alpha^{\prime \prime}$ and $\beta$ is acute. Interchanging $\alpha$ and $\beta$ if necessary, we may assume that the angle between the roots $\alpha^{\prime}+\beta^{\prime \prime}$ and $\alpha$ is acute. Then $\rho=\alpha-\left(\alpha^{\prime}+\beta^{\prime \prime}\right)=\alpha^{\prime \prime}-\beta^{\prime \prime} \in \Delta$. Again in view of the symmetry under the interchange of $\alpha$ and $\beta$ we can assume that $\rho \in \Delta_{+}$. Then $\alpha=\left(\alpha^{\prime}+\beta^{\prime \prime}\right)+\rho$, whence $\alpha^{\prime}+\beta^{\prime \prime} \in F(\alpha)$, $\delta=\pi(\alpha) \notin \operatorname{Supp}\left(\alpha^{\prime}+\beta^{\prime \prime}\right)$, and $\delta \notin \operatorname{Supp} \beta^{\prime \prime}$. On the other hand, $\beta=\beta^{\prime}+\beta^{\prime \prime}$ and therefore $\delta=\pi(\beta) \in \operatorname{Supp} \beta^{\prime \prime}$ (see Proposition 3). This gives a contradiction and shows that the angles between $\alpha^{\prime}+\beta^{\prime \prime}$ and $\alpha$ and between $\beta^{\prime}+\alpha^{\prime \prime}$ and $\beta$ are nonacute. We now prove that the angle between $\alpha^{\prime}+\beta^{\prime \prime}$ and $\beta^{\prime}+\alpha^{\prime \prime}$ is nonacute. If this is not the case, then $\rho=\alpha^{\prime}+\beta^{\prime \prime}-\beta^{\prime}-\alpha^{\prime \prime} \in \Delta$. Once again we can assume that $\rho \in \Delta_{+}$. Then $\alpha+\rho=2\left(\alpha^{\prime}+\beta^{\prime \prime}\right)-\beta \in \Psi$ and $\tau(\alpha+\rho)=\tau(\beta)=\tau\left(\alpha^{\prime}+\beta^{\prime \prime}\right)$ by Proposition 11. The roots $\alpha+\rho, \beta$, and $\alpha^{\prime}+\beta^{\prime \prime}$ are pairwise distinct and by Corollary 1 must be linearly 
independent, which is not the case. As a result of the preceding arguments we see that the four roots $\alpha, \beta, \alpha^{\prime}+\beta^{\prime \prime}, \beta^{\prime}+\alpha^{\prime \prime}$ are pairwise distinct and the angle between each pair is nonacute. Therefore, these roots are linearly independent by Lemma 1. On the other hand, there is the linear dependence $\alpha+\beta=\left(\alpha^{\prime}+\beta^{\prime \prime}\right)+\left(\beta^{\prime}+\alpha^{\prime \prime}\right)$. This contradiction proves the lemma.

Corollary 11. Suppose that $\alpha, \beta$ are distinct active roots such that $\tau(\alpha)=\tau(\beta)$ and $\pi(\alpha) \in \operatorname{Supp} \alpha \cap \operatorname{Supp} \beta$. Then $\pi(\alpha)=\pi(\beta)$.

Proof. By Corollary 6 there exists a root $\beta^{\prime} \in F(\beta)$ such that $\pi\left(\beta^{\prime}\right)=\pi(\alpha)$. Then in view of Lemma 10 we have $\tau\left(\beta^{\prime}\right)=\tau(\alpha)=\tau(\beta)$. By Lemma 5 we obtain $\beta^{\prime}=\beta$.

Lemma 11. Suppose that $\alpha, \beta$ are distinct active roots such that $\tau(\alpha)=\tau(\beta)$. Suppose that $\alpha^{\prime} \in F(\alpha), \beta^{\prime} \in F(\beta), \alpha^{\prime} \neq \beta^{\prime}$, and $\alpha-\alpha^{\prime} \neq \beta-\beta^{\prime}$. Then $\tau\left(\alpha^{\prime}\right) \neq \tau\left(\beta^{\prime}\right)$.

Proof. If $\alpha^{\prime}=\alpha$ or $\beta^{\prime}=\beta$, then the required assertion follows from Lemma 5 , Therefore in what follows we assume that $\alpha^{\prime} \neq \alpha$ and $\beta^{\prime} \neq \beta$. Then we have $\alpha=\alpha^{\prime}+\alpha^{\prime \prime}$ and $\beta=\beta^{\prime}+\beta^{\prime \prime}$ for some roots $\alpha^{\prime \prime}, \beta^{\prime \prime} \in \Delta_{+}$, and $\alpha^{\prime \prime} \neq \beta^{\prime \prime}$ by hypothesis. Assume that $\tau\left(\alpha^{\prime}\right)=\tau\left(\beta^{\prime}\right)$. Then, by Proposition 1, $\alpha^{\prime}+\beta^{\prime \prime}$ and $\beta^{\prime}+\alpha^{\prime \prime}$ are active roots and $\tau(\alpha)=\tau(\beta)=\tau\left(\alpha^{\prime}+\beta^{\prime \prime}\right)=\tau\left(\beta^{\prime}+\alpha^{\prime \prime}\right)$. Furthermore, the conditions $\alpha \neq \beta, \alpha^{\prime} \neq \beta^{\prime}$, and $\alpha^{\prime \prime} \neq \beta^{\prime \prime}$ imply that any two roots among $\alpha, \beta, \alpha^{\prime}+\beta^{\prime \prime}, \beta^{\prime}+\alpha^{\prime \prime}$ are distinct, apart from, possibly, the roots $\alpha^{\prime}+\beta^{\prime \prime}$ and $\beta^{\prime}+\alpha^{\prime \prime}$. In any case, by Corollary 1 all the (distinct) roots in the set $\left\{\alpha, \beta, \alpha^{\prime}+\beta^{\prime \prime}, \beta^{\prime}+\alpha^{\prime \prime}\right\}$ (which consists of three or four elements) are linearly independent. On the other hand, there is the linear dependence $\alpha+\beta=\left(\beta^{\prime}+\alpha^{\prime \prime}\right)+\left(\alpha^{\prime}+\beta^{\prime \prime}\right)$. We have obtained a contradiction, so $\tau\left(\alpha^{\prime}\right) \neq \tau\left(\beta^{\prime}\right)$.

Corollary 12. Let $\alpha, \beta$ be distinct active roots such that $\tau(\alpha)=\tau(\beta)$. Suppose that $\alpha^{\prime} \in F(\alpha) \backslash\{\alpha\}, \beta^{\prime} \in F(\beta) \backslash\{\beta\}, \alpha^{\prime} \neq \beta^{\prime}$, and $\tau\left(\alpha^{\prime}\right)=\tau\left(\beta^{\prime}\right)$. Then there exists a root $\gamma \in \Delta_{+}$such that $\alpha=\alpha^{\prime}+\gamma$ and $\beta=\beta^{\prime}+\gamma$.

Lemma 12. Let $\alpha, \beta$ be distinct active roots such that $\tau(\alpha)=\tau(\beta)$. Suppose that $\operatorname{Supp} \alpha \cap \operatorname{Supp} \beta=\{\gamma\}$, where $\gamma=\pi(\alpha)=\pi(\beta)$. Then $\alpha-\gamma \in F(\alpha)$ and $\beta-\gamma \in F(\beta)$.

Proof. By hypothesis, $F(\alpha) \cap F(\beta)=\varnothing$. Assume that for any two roots $\alpha^{\prime} \in F(\alpha) \backslash\{\alpha\}$ and $\beta^{\prime} \in F(\beta) \backslash\{\beta\}$ the condition $\tau\left(\alpha^{\prime}\right) \neq \tau\left(\beta^{\prime}\right)$ holds. Then, in view of Lemma 5 , the restriction to $S$ of the roots in $F(\alpha) \cup F(\beta)$ yields exactly $|F(\alpha)|+|F(\beta)|-1$ different weights. By Theorem 1 all these weights are linearly independent and generate a subspace $\Omega \subset \mathfrak{X}(S) \otimes_{\mathbb{Z}} \mathbb{Q}$ of dimension $|F(\alpha)|+|F(\beta)|-1$, which is equal to $|\operatorname{Supp} \alpha|+|\operatorname{Supp} \beta|-1$ by Lemma 7 (a). On the other hand, the subspace $\Omega$ is contained in the subspace $\Omega^{\prime}$ generated by the restrictions to $S$ of the roots in $\operatorname{Supp} \alpha \cup \operatorname{Supp} \beta$. The subspace $\Omega^{\prime}$ is generated by $|\operatorname{Supp} \alpha|+|\operatorname{Supp} \beta|-1$ elements, which satisfy the nontrivial relation $\tau(\alpha)=\tau(\beta)$. Hence, $\operatorname{dim} \Omega^{\prime} \leq|\operatorname{Supp} \alpha|+|\operatorname{Supp} \beta|-2$, a contradiction. Therefore there exist roots $\alpha^{\prime} \in F(\alpha) \backslash\{\alpha\}, \beta^{\prime} \in F(\beta) \backslash\{\beta\}$ such that $\tau\left(\alpha^{\prime}\right)=\tau\left(\beta^{\prime}\right)$. Then by Corollary 12 there exists a root $\delta \in \Delta_{+}$such that $\alpha=\alpha^{\prime}+\delta$ and $\beta=\beta^{\prime}+\delta$. It follows from the latter equalities that $\operatorname{Supp} \delta \subset \operatorname{Supp} \alpha \cap \operatorname{Supp} \beta=\{\gamma\}$. Hence, $\delta=\gamma$, $\alpha-\gamma=\alpha^{\prime} \in F(\alpha)$, and $\beta-\gamma=\beta^{\prime} \in F(\beta)$.

Proposition 5. Suppose that $\alpha, \beta$ are distinct active roots such that $\tau(\alpha)=\tau(\beta)$. Then exactly one of the possibilities (D0), (D1), (E1), (D2), (E2) is realized.

Proof. We can assume without loss of generality that $\Delta=\Delta \cap\langle\operatorname{Supp} \alpha \cup \operatorname{Supp} \beta\rangle$. Set $I=\operatorname{Supp} \alpha \cap \operatorname{Supp} \beta$. Assume that possibility (D0) is not realized and $I \neq \varnothing$. Then there exists a node $\delta \in I$ connected by an edge to a node contained in $\operatorname{Supp} \alpha \backslash I$ (the latter set is nonempty by Corollary [3(a) and Lemma [5(a)). There are three possible cases. 
Case 1. The root $\delta$ is terminal with respect to $\operatorname{Supp} \alpha$. Then $I=\{\delta\}$. By Corollary 8 the root $\delta$ is either an active root or the root associated with $\alpha$. If $\delta$ is an active root, then, by Corollary [7, $\delta$ is terminal with respect to $\operatorname{Supp} \beta$ and we have (D1). If $\delta=\pi(\alpha)$, then by Corollary 11 and Lemma 12 we obtain that $\delta=\pi(\beta), \alpha-\delta \in F(\alpha)$, and $\beta-\delta \in F(\beta)$. We now show that $\delta$ is terminal with respect to $\operatorname{Supp} \beta$. Consider the degree $d$ of the node $\delta$ in the diagram $\Sigma(\Pi)$. If $d=2$, then $\delta$ is automatically terminal with respect to $\operatorname{Supp} \beta$. If $d=3$, then the root system $\Delta(\beta)$ has type A, and so by Theorem 3 the root $\beta$ is equal to the sum of all roots in $\operatorname{Supp} \beta$. But then the support of the $\operatorname{root} \beta-\delta$ is disconnected, which is impossible. Therefore, $d=2, \delta$ is terminal with respect to $\operatorname{Supp} \beta$, and (E1) is realized.

Case 2. The root $\delta$ is not terminal with respect to $\operatorname{Supp} \alpha$ but is terminal with respect to $\operatorname{Supp} \beta$. If $I=\{\delta\}$, then we can interchange $\alpha$ and $\beta$ and consider Case 1 . Therefore we assume that $I \neq\{\delta\}$. Let $\delta^{\prime}$ be the node of the diagram $\Sigma(I)$ connected by an edge to $\delta$. By Corollaries 7 , 8, and 11 we have $\delta=\pi(\beta)=\pi(\alpha)$. Let roots $\alpha^{\prime} \in F(\alpha) \backslash\{\alpha\}$ and $\beta^{\prime} \in F(\beta) \backslash\{\beta\}$ be such that $\pi\left(\alpha^{\prime}\right)=\pi\left(\beta^{\prime}\right)=\delta$. By Lemma 10 we obtain that $\tau\left(\alpha^{\prime}\right)=\tau\left(\beta^{\prime}\right)$. If $\alpha^{\prime} \neq \beta^{\prime}$, then by Corollary 12 we have $\alpha-\alpha^{\prime}=\beta-\beta^{\prime}$ and, in particular, $\operatorname{Supp}\left(\alpha-\alpha^{\prime}\right) \subset I$. Since $\delta \notin \operatorname{Supp} \alpha^{\prime}$ and $\delta^{\prime} \in \operatorname{Supp} \alpha^{\prime}$, the set $\operatorname{Supp}\left(\alpha-\alpha^{\prime}\right)$ contains a node of the set Supp $\alpha \backslash I$ connected by an edge to $\delta$, a contradiction. If, however, $\alpha^{\prime}=\beta^{\prime}$, then in view of Corollary 9 we obtain that $\operatorname{Supp} \beta \subset I$, which is also impossible.

Case 3. The root $\delta$ is terminal with respect to neither $\operatorname{Supp} \alpha$ nor $\operatorname{Supp} \beta$. Arguing just as in case 2 of the proof of Proposition 4, we obtain that the diagram $\Sigma(\Pi)$ has the form depicted in Figure 1 (for some $p, q, r \geq 1$ ) and further, $\alpha=\alpha_{1}+\cdots+\alpha_{p}+\gamma_{0}+\gamma_{1}+\cdots+\gamma_{r}$, $\beta=\beta_{1}+\cdots+\beta_{q}+\gamma_{0}+\gamma_{1}+\cdots+\gamma_{r}, \delta=\gamma_{0}$. In this situation, in view of Corollary 11 we obtain that one of the possibilities (D2) or (E2) holds.

3.3. The main goal of this subsection is to prove the following proposition.

Proposition 6. Let $\alpha$ be a maximal active root. Then there exists a simple root $\widetilde{\alpha} \in$ $\operatorname{Supp} \alpha$ such that $\widetilde{\alpha} \notin \operatorname{Supp} \beta$ for any maximal active root $\beta \neq \alpha$. In other words, the support of a maximal active root is not entirely covered by the supports of other maximal active roots.

Before we prove this proposition, let us prove several auxiliary lemmas.

Lemma 13. Suppose that $\alpha, \beta$ are distinct active roots such that $\tau(\alpha)=\tau(\beta)$ and $\pi(\alpha)=\pi(\beta)$.

(a) There exists a unique node $\eta(\alpha, \beta) \in \operatorname{Supp} \alpha \backslash \operatorname{Supp} \beta$ of the diagram $\Sigma(\Pi)$ connected by an edge to a node in $\operatorname{Supp} \alpha \cap \operatorname{Supp} \beta$.

(b) If a root $\alpha^{\prime} \in F(\alpha)$ is such that $\pi\left(\alpha^{\prime}\right)=\eta(\alpha, \beta)$, then there exists a root $\beta^{\prime} \in F(\beta)$ with $\tau\left(\alpha^{\prime}\right)=\tau\left(\beta^{\prime}\right)$.

Proof. It follows from Proposition 5 that exactly one of the possibilities (E1) or (E2) is realized for $\alpha, \beta$. It is easy to see that part (a) holds in either case. Let us prove part (b). We consider the two possibilities separately.

Case 1. Possibility (E1) is realized. Then in view of Lemma 12 the root $\alpha$ is contained in either row 1 or row 2 of Table 1 . We denote by $\delta$ the unique simple root contained in $\operatorname{Supp} \alpha \cap \operatorname{Supp} \beta$. Then the root $\alpha^{\prime}=\alpha-\delta$ is the required one.

Case 2. Possibility (E2) is realized. Then the diagram $\Sigma(\operatorname{Supp} \alpha \cup \operatorname{Supp} \beta)$ has the form depicted in Figure 1 (for some $p, q, r \geq 1$ ), $\alpha=\alpha_{1}+\cdots+\alpha_{p}+\gamma_{0}+\gamma_{1}+\cdots+\gamma_{r}$, $\beta=\beta_{1}+\cdots+\beta_{q}+\gamma_{0}+\gamma_{1}+\cdots+\gamma_{r}$, and $\pi(\alpha)=\pi(\beta)=\gamma_{s}$, where $0 \leq s \leq r$. Then, obviously, $\alpha^{\prime}=\alpha_{1}+\cdots+\alpha_{p}$ and $\beta^{\prime}=\beta_{1}+\cdots+\beta_{q}$. 
Lemma 14. Suppose that $\alpha, \beta, \gamma$ are pairwise distinct active roots such that $\tau(\alpha)=$ $\tau(\beta)=\tau(\gamma)$ and $\pi(\alpha)=\pi(\beta)=\pi(\gamma)$. Then either $\operatorname{Supp} \alpha \cap \operatorname{Supp} \beta \subset \operatorname{Supp} \alpha \cap \operatorname{Supp} \gamma$ or $\operatorname{Supp} \alpha \cap \operatorname{Supp} \gamma \subset \operatorname{Supp} \alpha \cap \operatorname{Supp} \beta$.

Proof. By Proposition 5 one of the possibilities (E1) or (E2) is realized for each of the pairs $\alpha, \beta$ and $\alpha, \gamma$. If possibility (E1) is realized for at least one of these pairs, then the assertion holds. It remains to observe that possibility (E2) cannot be realized simultaneously for both of these pairs.

Lemma 15. Let $\alpha$ be a maximal active root. Suppose that a maximal active root $\beta \neq \alpha$ is such that $\pi(\alpha) \in \operatorname{Supp} \alpha \cap \operatorname{Supp} \beta$ and the set $\operatorname{Supp} \alpha \cap \operatorname{Supp} \beta$ is maximal with respect to inclusion. Then for every maximal active root $\gamma$ distinct from $\alpha$ we have $\eta(\alpha, \beta) \notin \operatorname{Supp} \gamma$.

Proof. By Corollaries 10 and 11 we have $\tau(\alpha)=\tau(\beta)$ and $\pi(\alpha)=\pi(\beta)$. Consider the root $\eta=\eta(\alpha, \beta)$. By definition we have $\eta \notin \operatorname{Supp} \beta$. Let a root $\alpha^{\prime} \in F(\alpha) \backslash\{\alpha\}$ be such that $\pi\left(\alpha^{\prime}\right)=\eta$. Then by Lemma 13(b) there exists a root $\beta^{\prime} \in F(\beta) \backslash\{\beta\}$ with $\tau\left(\alpha^{\prime}\right)=\tau\left(\beta^{\prime}\right)$. By Corollary 12 there exists a root $\delta \in \Delta_{+}$such that $\delta=\alpha-\alpha^{\prime}=\beta-\beta^{\prime}$.

Assume that $\eta$ is contained in the support of a maximal active root $\gamma$ distinct from $\alpha$ and $\beta$. Since $\eta \in \operatorname{Supp} \alpha \cap \operatorname{Supp} \gamma$ and $\pi(\alpha) \neq \eta$, we have $\pi(\gamma) \neq \eta$. Consider a root $\gamma^{\prime} \in F(\gamma) \backslash\{\gamma\}$ such that $\pi\left(\gamma^{\prime}\right)=\eta$ and let $\gamma^{\prime \prime}=\gamma-\gamma^{\prime} \in \Delta_{+}$. By Lemma 10 we have $\tau\left(\gamma^{\prime}\right)=\tau\left(\alpha^{\prime}\right)$. If $\gamma^{\prime \prime}=\delta$, then $\tau(\gamma)=\tau(\alpha)=\tau(\beta)$, so that $\pi(\gamma) \in \operatorname{Supp} \delta \subset$ $\operatorname{Supp} \alpha \cap \operatorname{Supp} \gamma$, and $\pi(\gamma)=\pi(\alpha)$ by Corollary 11. Since $\eta \in(\operatorname{Supp} \alpha \cap \operatorname{Supp} \gamma) \backslash \operatorname{Supp} \beta$, by Lemma 14 we obtain that $\operatorname{Supp} \alpha \cap \operatorname{Supp} \gamma \supsetneqq \operatorname{Supp} \alpha \cap \operatorname{Supp} \beta$, which contradicts the choice of $\beta$. Therefore, $\gamma^{\prime \prime} \neq \delta$. Next, by Proposition [1, $\alpha^{\prime}+\gamma^{\prime \prime}, \beta^{\prime}+\gamma^{\prime \prime}$, and $\gamma^{\prime}+\delta$ are maximal active roots. By Lemma 3 all distinct roots in the set $\left\{\alpha, \beta, \gamma, \alpha^{\prime}+\right.$ $\left.\gamma^{\prime \prime}, \beta^{\prime}+\gamma^{\prime \prime}, \gamma^{\prime}+\delta\right\}$ are linearly independent. However, these roots satisfy the relation $\alpha+\beta+2 \gamma=\left(\alpha^{\prime}+\gamma^{\prime \prime}\right)+\left(\beta^{\prime}+\gamma^{\prime \prime}\right)+2\left(\gamma^{\prime}+\delta\right)$. Since the root $\gamma$ is different from $\alpha$, $\beta, \beta^{\prime}+\gamma^{\prime \prime}, \gamma^{\prime}+\delta$, this relation is nontrivial. We have arrived at a contradiction, which proves the lemma.

Proof of Proposition 6. If $\pi(\alpha)$ is not contained in the support of any maximal active root different from $\alpha$, then we can take $\widetilde{\alpha}=\pi(\alpha)$. Otherwise we have $\pi(\alpha) \in \operatorname{Supp} \beta$ for some maximal active root $\beta \neq \alpha$. We assume without loss of generality that the set $\operatorname{Supp} \alpha \cap \operatorname{Supp} \beta$ is maximal with respect to inclusion. Then by Lemma 15 we can take $\widetilde{\alpha}=\eta(\alpha, \beta)$.

3.4. In this subsection we describe a condition relating the torus $S$ with the set $\Psi$. The main result is stated in Proposition 7.

Recall (see Corollary 6) that the map $\pi: F(\alpha) \rightarrow \operatorname{Supp} \alpha$ is bijective for every active $\operatorname{root} \alpha$.

Lemma 16. Let $\alpha, \beta$ be distinct maximal active roots. Let $J=\operatorname{Supp} \alpha \backslash \operatorname{Supp} \beta$.

(a) If $\tau(\alpha) \neq \tau(\beta)$, then for every root $\alpha^{\prime} \in F(\alpha)$ with $\pi\left(\alpha^{\prime}\right) \in J$ and for every root $\beta^{\prime} \in F(\beta)$ we have $\tau\left(\alpha^{\prime}\right) \neq \tau\left(\beta^{\prime}\right)$.

(b) If $\tau(\alpha)=\tau(\beta)$ and $\pi(\alpha) \in J$, then for every root $\alpha^{\prime} \in F(\alpha) \backslash\{\alpha\}$ with $\pi\left(\alpha^{\prime}\right) \in J$ and for every root $\beta^{\prime} \in F(\beta)$ we have $\tau\left(\alpha^{\prime}\right) \neq \tau\left(\beta^{\prime}\right)$.

(c) If $\tau(\alpha)=\tau(\beta)$ and $\pi(\alpha) \in \operatorname{Supp} \alpha \cap \operatorname{Supp} \beta$, then for every root $\alpha^{\prime} \in F(\alpha)$ with $\pi\left(\alpha^{\prime}\right) \in J \backslash\{\eta(\alpha, \beta)\}$ and for every root $\beta^{\prime} \in F(\beta)$ we have $\tau\left(\alpha^{\prime}\right) \neq \tau\left(\beta^{\prime}\right)$.

Proof. Part (a) is an immediate consequence of Lemma 8. We now prove part (b). Let the roots $\alpha^{\prime} \in F(\alpha) \backslash\{\alpha\}$ and $\beta^{\prime} \in F(\beta)$ be such that $\pi\left(\alpha^{\prime}\right) \in J$ and $\tau\left(\alpha^{\prime}\right)=\tau\left(\beta^{\prime}\right)$. Then by Lemma 11 we obtain that $\delta=\alpha-\alpha^{\prime}=\beta-\beta^{\prime} \in \Delta_{+}$. Hence, $\pi(\alpha) \in \operatorname{Supp} \delta \subset \operatorname{Supp} \alpha \backslash J$, a contradiction. Under the hypotheses of part (c), by Proposition 5 one of the possibilities (E1) or (E2) is realized for $\alpha, \beta$. In both cases, as is easy to see, every root $\alpha^{\prime} \in F(\alpha)$ with 
$\pi\left(\alpha^{\prime}\right) \in J \backslash\{\eta(\alpha, \beta)\}$ is subordinate to the root $\alpha^{\prime \prime} \in F(\alpha)$ such that $\pi\left(\alpha^{\prime \prime}\right)=\eta(\alpha, \beta)$. Assuming that $\tau\left(\alpha^{\prime}\right)=\tau\left(\beta^{\prime}\right)$ for some $\operatorname{root} \beta^{\prime} \in F(\beta)$, by Lemma 11 we obtain that $\delta=\alpha-\alpha^{\prime}=\beta-\beta^{\prime} \in \Delta_{+}$. Then $\eta(\alpha, \beta) \in \operatorname{Supp} \delta$, which is impossible because $\operatorname{Supp} \delta \subset \operatorname{Supp} \alpha \cap \operatorname{Supp} \beta$.

We denote by $\mathrm{M}=\mathrm{M}(H)$ the set of maximal active roots of the subgroup $H$.

Lemma 17. Let $\mathrm{M}^{\prime} \subset \mathrm{M}$ be an arbitrary subset. Set $l=\left|\bigcup_{\delta \in \mathrm{M}^{\prime}} \operatorname{Supp} \delta\right|$ and $k=$ $\left|\tau\left(\bigcup_{\delta \in \mathrm{M}^{\prime}} F(\delta)\right)\right|$. Then:

(a) $\operatorname{dim}\left\langle\mu-\nu \mid \mu, \nu \in \mathrm{M}^{\prime}, \tau(\mu)=\tau(\nu)\right\rangle=\left|\mathrm{M}^{\prime}\right|-\left|\tau\left(\mathrm{M}^{\prime}\right)\right|$;

(b) $l=k+\left|\mathrm{M}^{\prime}\right|-\left|\tau\left(\mathrm{M}^{\prime}\right)\right|$.

Proof. We prove both parts by simultaneous induction on $\left|\mathrm{M}^{\prime}\right|$.

For $\left|\mathrm{M}^{\prime}\right|=1$ we have $\left|\mathrm{M}^{\prime}\right|=\left|\tau\left(\mathrm{M}^{\prime}\right)\right|$, and part (a) is obviously true. Part (b) is also true by Lemmas 5 and 7 (a).

We now assume that parts (a) and (b) hold for all proper subsets of $\mathrm{M}^{\prime}$ and prove these assertions for $\mathrm{M}^{\prime}$. Let $\mathrm{M}^{\prime}=\widetilde{\mathrm{M}}^{\prime} \cup\{\alpha\}$, where $\alpha \notin \widetilde{\mathrm{M}}^{\prime}$. Set $J=(\operatorname{Supp} \alpha) \backslash\left(\bigcup_{\delta \in \widetilde{\mathrm{M}}^{\prime}} \operatorname{Supp} \delta\right)$. Set also $\tilde{l}=\left|\bigcup_{\delta \in \widetilde{\mathrm{M}}^{\prime}} \operatorname{Supp} \delta\right|$ and $\widetilde{k}=\left|\tau\left(\bigcup_{\delta \in \widetilde{\mathrm{M}}^{\prime}} F(\delta)\right)\right|$. Clearly, $\left|\mathrm{M}^{\prime}\right|=\left|\mathrm{M}^{\prime}\right|+1$ and $l=\widetilde{l}+|J|$. We point out the following two properties of $\alpha$ :

(1) if $\alpha^{\prime}, \alpha^{\prime \prime} \in F(\alpha)$ are distinct roots, then $\tau\left(\alpha^{\prime}\right) \neq \tau\left(\alpha^{\prime \prime}\right)$ (see Lemma 5);

(2) if for a root $\alpha^{\prime} \in F(\alpha)$ it happens that $\pi\left(\alpha^{\prime}\right) \in \operatorname{Supp} \beta$ for some $\beta \in \widetilde{\mathrm{M}}^{\prime}$, then there exists a root $\beta^{\prime} \in F(\beta)$ such that $\tau\left(\alpha^{\prime}\right)=\tau\left(\beta^{\prime}\right)$ (this follows from Lemma 10).

Further we consider two cases.

Case 1. For every root $\delta \in \widetilde{\mathrm{M}}^{\prime}$ we have $\tau(\alpha) \neq \tau(\delta)$. Then $\left|\tau\left(\mathrm{M}^{\prime}\right)\right|=\left|\tau\left(\widetilde{\mathrm{M}}^{\prime}\right)\right|+1$ and the subspace $\left\langle\mu-\nu \mid \mu, \nu \in \mathrm{M}^{\prime}, \tau(\mu)=\tau(\nu)\right\rangle$ coincides with the subspace $\langle\mu-\nu| \mu, \nu \in$ $\left.\widetilde{\mathrm{M}}^{\prime}, \tau(\mu)=\tau(\nu)\right\rangle$, which has dimension $\left|\widetilde{\mathrm{M}}^{\prime}\right|-\left|\tau\left(\widetilde{\mathrm{M}}^{\prime}\right)\right|=\left|\mathrm{M}^{\prime}\right|-\left|\tau\left(\mathrm{M}^{\prime}\right)\right|$ by the induction hypothesis. We have thus proved part (a). To prove part (b), by the induction hypothesis it suffices to verify that $|J|=k-\widetilde{k}$. By Lemma 16(a), for every root $\alpha^{\prime} \in F(\alpha)$ with $\pi\left(\alpha^{\prime}\right) \in J$ and every root $\beta \in \bigcup_{\delta \in \widetilde{\mathrm{M}}^{\prime}} F(\delta)$ we have $\tau\left(\alpha^{\prime}\right) \neq \tau(\beta)$. Hence, in view of properties (1) and (2), we obtain $|J|=k-\widetilde{k}$.

Case 2. There exists a root $\alpha_{0} \in \widetilde{\mathrm{M}}^{\prime}$ such that $\tau(\alpha)=\tau\left(\alpha_{0}\right)$. Then $\left|\tau\left(\mathrm{M}^{\prime}\right)\right|=\left|\tau\left(\widetilde{\mathrm{M}}^{\prime}\right)\right|$. By Proposition 6 there exists a simple root $\rho \in \operatorname{Supp} \alpha$ with $\rho \in J$, whence $\alpha-\alpha_{0}$ is not contained in the subspace $\left\langle\mu-\nu \mid \mu, \nu \in \widetilde{\mathrm{M}}^{\prime}, \tau(\mu)=\tau(\nu)\right\rangle$. It is easy to see that $\left\langle\mu-\nu \mu, \nu \in \mathrm{M}^{\prime}, \tau(\mu)=\tau(\nu)\right\rangle$ coincides with the subspace $\left\langle\mu-\nu \mu, \nu \in \widetilde{\mathrm{M}}^{\prime}, \tau(\mu)=\right.$ $\tau(\nu)\rangle \oplus\left\langle\alpha-\alpha_{0}\right\rangle$, and its dimension is equal to $\left|\widetilde{\mathrm{M}}^{\prime}\right|-\left|\tau\left(\widetilde{\mathrm{M}}^{\prime}\right)\right|+1=\left|\mathrm{M}^{\prime}\right|-\left|\tau\left(\mathrm{M}^{\prime}\right)\right|$ by the induction hypothesis. Part (a) is proved. To prove part (b), by the induction hypothesis it suffices to verify that $|J|=k-\widetilde{k}+1$. We consider two subcases.

Subcase 2.1. $\pi(\alpha) \in J$. By Lemma 16(a, b), for every root $\alpha^{\prime} \in F(\alpha)$ such that $\pi\left(\alpha^{\prime}\right) \in J \backslash\{\pi(\alpha)\}$ and for every $\operatorname{root} \beta \in \bigcup_{\delta \in \widetilde{\mathrm{M}}^{\prime}} F(\delta)$ we have $\tau\left(\alpha^{\prime}\right) \neq \tau(\beta)$. Hence by properties (1) and (2) we obtain $|J|=k-\widetilde{k}+1$.

Subcase 2.2. $\pi(\alpha) \notin J$. In this situation there exists a maximal active $\operatorname{root} \beta \neq \alpha$ such that $\pi(\alpha) \in \operatorname{Supp} \beta$. We assume without loss of generality that the set $\operatorname{Supp} \alpha \cap \operatorname{Supp} \beta$ is maximal with respect to inclusion. Then by Lemma 15 we have $\eta(\alpha, \beta) \in J$. Let $\alpha^{\prime} \in F(\alpha)$ be the root such that $\pi\left(\alpha^{\prime}\right)=\eta(\alpha, \beta)$. Lemma 13) (b) implies that there exists a root $\beta^{\prime} \in F(\beta)$ such that $\tau\left(\alpha^{\prime}\right)=\tau\left(\beta^{\prime}\right)$. Assume that for some root $\alpha^{\prime \prime} \in F(\alpha)$ with $\pi\left(\alpha^{\prime \prime}\right) \in J \backslash\{\eta(\alpha, \beta)\}$ there exist roots $\gamma \in \widetilde{\mathrm{M}}^{\prime}$ and $\gamma^{\prime} \in F(\gamma)$ such that $\tau\left(\alpha^{\prime \prime}\right)=\tau\left(\gamma^{\prime}\right)$. Clearly, $\alpha^{\prime \prime} \neq \gamma^{\prime}$. We set $\eta^{\prime}=\pi\left(\alpha^{\prime \prime}\right)$. Applying Lemmas 8 and 11 we find that $\tau(\alpha)=$ $\tau(\gamma)$ and $\alpha-\alpha^{\prime \prime}=\gamma-\gamma^{\prime} \in \Delta_{+}$, whence $\pi(\alpha) \in \operatorname{Supp} \alpha \cap \operatorname{Supp} \gamma$. Then $\eta^{\prime}=\eta(\alpha, \gamma)$ by Lemma 16)(c). Therefore, in the diagram $\Sigma(\Pi)$ the node $\eta^{\prime}$ is connected by an edge to a node in $\operatorname{Supp} \alpha \cap \operatorname{Supp} \gamma$. Next, by Corollary 11 we have $\pi(\alpha)=\pi(\gamma)$. By the choice of $\beta$ and by Lemma 14 we have $\operatorname{Supp} \alpha \cap \operatorname{Supp} \gamma \subset \operatorname{Supp} \alpha \cap \operatorname{Supp} \beta$. Hence 
in the diagram $\Sigma(\Pi)$ the node $\eta^{\prime} \in \operatorname{Supp} \alpha \backslash \operatorname{Supp} \beta$ is connected to a node in the set $\operatorname{Supp} \alpha \cap \operatorname{Supp} \beta$. Then by Lemma 13(a) we have $\eta^{\prime}=\eta(\alpha, \beta)$, which is impossible. Thus, for every root $\alpha^{\prime \prime} \in F(\alpha)$ with $\pi\left(\alpha^{\prime}\right) \in J \backslash\{\eta(\alpha, \beta)\}$ and every root $\gamma^{\prime} \in \bigcup_{\delta \in \widetilde{\mathrm{M}}^{\prime}} F(\delta)$ we have $\tau\left(\alpha^{\prime \prime}\right) \neq \tau\left(\gamma^{\prime}\right)$. Hence by properties (1) and (2) we obtain $|J|=k-\widetilde{k}+1$.

Part (b) is proved.

Proposition 7. The kernel of the map $\tau:\left\langle\bigcup_{\delta \in \mathrm{M}} \operatorname{Supp} \delta\right\rangle \rightarrow \mathfrak{X}(S) \otimes_{\mathbb{Z}} \mathbb{Q}$ coincides with the subspace $\langle\mu-\nu \mid \mu, \nu \in \mathrm{M}, \tau(\mu)=\tau(\nu)\rangle$.

Proof. Set $R=\left\langle\bigcup_{\delta \in \mathrm{M}} \operatorname{Supp} \delta\right\rangle \subset Q$. By Theorem 1 the dimension of $\tau(R)$ is at least $K$. Next, since $\left.\langle\mu-\nu \mid \mu, \nu \in \mathrm{M}, \tau(\mu)=\tau(\nu)\rangle \subset \operatorname{Ker} \tau\right|_{R}$, it follows from Lemma 17(a) that $\left.\operatorname{dim} \operatorname{Ker} \tau\right|_{R} \geq|\mathrm{M}|-|\tau(\mathrm{M})|$. Applying Lemma 17(b) we find that $\left.\operatorname{dim} \operatorname{Ker} \tau\right|_{R}=$ $|\mathrm{M}|-|\tau(\mathrm{M})|$, giving the required assertion.

3.5. Here we summarize the results obtained in $\S 3$ and prove the uniqueness theorem (see Theorem 4).

Recall that in $\S 3.4$ we introduced the notation $\mathrm{M}=\mathrm{M}(H)$ for the set of maximal active roots of $H$. We now introduce a relation $\sim$ on $\mathrm{M}$ as follows. For any two roots $\alpha, \beta \in \mathrm{M}$ we set $\alpha \sim \beta$ if and only if $\tau(\alpha)=\tau(\beta)$. Obviously, this is an equivalence relation.

With every connected solvable spherical subgroup $H \subset G$ standardly embedded in $B$ we associate the set of combinatorial data $\Upsilon(H)=(S, \mathrm{M}, \pi, \sim)$. We also set $\Upsilon_{0}(H)=$ $(\mathrm{M}, \pi, \sim)$. (In each of the sets $\Upsilon(H)$ and $\Upsilon_{0}(H), \pi$ is considered as a map from M to $\Pi$.)

Theorem 4 (Uniqueness). Let $H \subset G$ be a connected solvable spherical subgroup standardly embedded in $B$. Then, up to a conjugation by an element of $T, H$ is uniquely determined by its set of combinatorial data $\Upsilon(H)=(S, \mathrm{M}, \pi, \sim)$, and this set satisfies the following conditions:

(A) $\pi(\alpha) \in \operatorname{Supp} \alpha$ for every root $\alpha \in \mathrm{M}$, and the pair $(\alpha, \pi(\alpha))$ is contained in Table 1 .

(D) if $\alpha, \beta \in \mathrm{M}$ and $\alpha \nsim \beta$, then one of the possibilities (D0), (D1), (D2) is realized for $\alpha, \beta$;

(E) if $\alpha, \beta \in \mathrm{M}$ and $\alpha \sim \beta$, then one of the possibilities (D0), (D1), (E1), (D2), (E2) is realized for $\alpha, \beta$;

(C) if $\alpha \in \mathrm{M}$, then $\operatorname{Supp} \alpha \not \subset \bigcup_{\delta \in \mathrm{M} \backslash\{\alpha\}} \operatorname{Supp} \delta$;

(T) $\left.\operatorname{Ker} \tau\right|_{R}=\langle\mu-\nu \mid \mu, \nu \in \mathrm{M}, \mu \sim \nu\rangle$, where $R=\left\langle\bigcup_{\delta \in \mathrm{M}} \operatorname{Supp} \delta\right\rangle$.

Proof. By Corollary 5 the set $\Psi$ is uniquely determined by the pair $(\mathrm{M}, \pi)$. Then by Theorem 2, up to a conjugation by an element of $T, H$ is uniquely determined by the triple $(S, \mathrm{M}, \pi)$.

Condition (A) follows from the definition of the root $\pi(\alpha)$ and from Theorem 3 , Conditions (D) and (E) follow from Propositions 4 and 5, respectively. The validity of condition (C) was established in Proposition 6. Finally, the fact that condition (T) holds was proved in Proposition 7 .

Remark 2. The set of data $(S, \mathrm{M}, \pi, \sim)$ is redundant in the sense that the relation $\sim$ is uniquely determined by the torus $S$ and the set M. However, the advantage of this set consists in the fact that, as we shall see in $\S$, the unipotent radical $N$ of $H$ can be constructed using only the subset $(\mathrm{M}, \pi, \sim)$ with no need of $S$ (see Remark 4).

Remark 3. If two distinct connected solvable spherical subgroups $H_{1}, H_{2} \subset G$ standardly embedded in $B$ are conjugate in $G$, then, generally speaking, the sets of combinatorial data $(S, \mathrm{M}, \pi, \sim)$ corresponding to them are different. Thus, in general, the set 
$(S, \mathrm{M}, \pi, \sim)$ is not an invariant of the conjugacy classes of connected solvable spherical subgroups. We shall return to this question in $\S 5$.

\section{$\S 4$. EXISTENCE THEOREM}

In this section we show that, given a set of combinatorial data described in the statement of Theorem 4, one can construct a connected solvable spherical subgroup of $G$ standardly embedded in $B$ with this set of combinatorial data. Namely, we shall prove the following theorem.

Theorem 5 (Existence). Suppose that a subtorus $S \subset T$, a subset $\mathrm{M} \subset \Delta_{+}$, a map $\pi: \mathrm{M} \rightarrow \Pi$, and an equivalence relation $\sim$ on $\mathrm{M}$ satisfy conditions $(\mathrm{A}),(\mathrm{D}),(\mathrm{E}),(\mathrm{C})$, and $(\mathrm{T})$. Then there exists a connected solvable spherical subgroup $H \subset G$ standardly embedded in $B$ such that $\Upsilon(H)=(S, \mathrm{M}, \pi, \sim)$.

In $\S 4.1$ we present some preliminary information that will be needed in the proof. The proof itself is carried out in $\S \S 4.24 .4$.

4.1. Suppose that a pair $\left(\alpha, \alpha_{0}\right)$, where $\alpha \in \Delta_{+}, \alpha_{0} \in \operatorname{Supp} \alpha$, is such that the root $\alpha$ is contained in column " $\alpha$ " of Table 1] and the root $\alpha_{0}$ is contained in the same row in column " $\pi(\alpha)$ " of this table. We set

$$
F(\alpha)=\{\alpha\} \cup\left\{\alpha^{\prime} \in \Delta_{+} \mid \alpha-\alpha^{\prime} \in \Delta_{+}, \alpha_{0} \notin \operatorname{Supp} \alpha^{\prime}\right\} .
$$

Then an easy case-by-case analysis establishes that the following properties hold:

(1) if $\beta \in F(\alpha)$, then $\beta$ is contained in Table 1

(2) if $\beta \in F(\alpha)$ and $\beta=\beta_{1}+\beta_{2}$ for some roots $\beta_{1}, \beta_{2} \in \Delta_{+}$, then exactly one of the two roots $\beta_{1}, \beta_{2}$ is contained in $F(\alpha)$;

(3) for every $\beta \in F(\alpha)$ we have $\left|\{\beta\} \cup\left\{\beta^{\prime} \in F(\alpha) \mid \beta-\beta^{\prime} \in \Delta_{+}\right\}\right|=|\operatorname{Supp} \beta|$; in particular, $|F(\alpha)|=|\operatorname{Supp} \alpha|$;

(4) all roots in the set $F(\alpha)$ are linearly independent (in view of (3), this is equivalent to the equality $\langle F(\alpha)\rangle=\langle\operatorname{Supp} \alpha\rangle)$.

4.2. We proceed to the proof of Theorem 5. Suppose that a set of combinatorial data ( $S, \mathrm{M}, \pi, \sim)$, where $S \subset T$ is a subtorus, $\mathrm{M} \subset \Delta_{+}$is a subset, $\pi: \mathrm{M} \rightarrow \Pi$ is a map, and $\sim$ is an equivalence relation on $\mathrm{M}$, satisfies conditions $(\mathrm{A}),(\mathrm{D}),(\mathrm{E}),(\mathrm{C})$, and $(\mathrm{T})$.

For each pair $(\alpha, \pi(\alpha))$, where $\alpha \in \mathrm{M}$, we construct the set $F(\alpha)$ as described in $\S 4.1$, and form the set $\Psi=\bigcup_{\alpha \in \mathrm{M}} F(\alpha)$.

In this subsection we derive basic properties of the set $\Psi$ which are necessary for the proof of Theorem 5

Lemma 18. Suppose that roots $\alpha \in \mathrm{M}$ and $\beta \in \Psi$ are such that $\operatorname{Supp} \beta \subset \operatorname{Supp} \alpha$. Then $\beta \in F(\alpha)$.

Proof. Consider a root $\widetilde{\beta} \in \mathrm{M}$ such that $\beta \in F(\widetilde{\beta})$. If $\widetilde{\beta}=\alpha$, then there is nothing to prove; therefore we assume that $\widetilde{\beta} \neq \alpha$. By conditions (D) and (E), one of the possibilities (D1), (E1), (D2), or (E2) is realized for the roots $\alpha, \widetilde{\beta}$. A direct check in each of these cases shows that the assertion is true.

Lemma 19. Suppose that $\alpha \in \Psi$ and $\alpha=\alpha_{1}+\alpha_{2}$ for some roots $\alpha_{1}, \alpha_{2} \in \Delta_{+}$. Then exactly one of the two roots $\alpha_{1}, \alpha_{2}$ is contained in $\Psi$.

Proof. Let a root $\widetilde{\alpha} \in \mathrm{M}$ (possibly, coinciding with $\alpha$ ) be such that $\alpha \in F(\widetilde{\alpha})$. Then by property (2) exactly one of the two roots $\alpha_{1}, \alpha_{2}$ is contained in $F(\widetilde{\alpha})$. Suppose, for definiteness, that $\alpha_{1} \in F(\widetilde{\alpha})$. If $\alpha_{2} \in \Psi$, then by Lemma 18 we obtain $\alpha_{2} \in F(\widetilde{\alpha})$, which is not the case. 
We now define the set $F(\alpha)$ for an arbitrary root $\alpha \in \Psi: F(\alpha)=\{\alpha\} \cup\left\{\alpha^{\prime} \in \Psi\right.$ $\left.\alpha-\alpha^{\prime} \in \Delta_{+}\right\}$. For roots $\alpha \in \mathrm{M}$ this definition coincides with the one given above.

Corollary 13. Let $\alpha \in \Psi$ be an arbitrary root. Then:

(a) $|F(\alpha)|=|\operatorname{Supp} \alpha|$;

(b) all roots in $F(\alpha)$ are linearly independent (in view of (a), this is equivalent to the equality $\langle F(\alpha)\rangle=\langle\operatorname{Supp} \alpha\rangle)$.

Proof. Part (a) follows from property (3) and Lemma 19. part (b) follows from property (4).

Proposition 8. (a) Suppose that $\alpha \in \Psi$. Then there exists a unique simple root $\pi(\alpha) \in$ Supp $\alpha$ with the following property: if $\alpha=\alpha_{1}+\alpha_{2}$ for some roots $\alpha_{1}, \alpha_{2} \in \Delta_{+}$, then $\alpha_{1}$ (resp. $\alpha_{2}$ ) belongs to $\Psi$ if and only if $\pi(\alpha) \notin \operatorname{Supp} \alpha_{1}$ (resp. $\left.\pi(\alpha) \notin \operatorname{Supp} \alpha_{2}\right)$.

(b) For every root $\alpha \in \Psi$, the map $\pi: F(\alpha) \rightarrow \operatorname{Supp} \alpha$ is a bijection.

Proof. The proofs of parts (a) and (b) repeat almost word-for-word the proofs of Proposition 3 and Corollary 6. respectively, the only difference being that the references to Lemma [(a) and Corollary 2 are replaced by the references to Corollaries 13(a) and 13(b), respectively.

Thus, we have defined the map $\pi$ on the entire set $\Psi$. Note that on the set $\mathrm{M}$ this map coincides with the given map $\pi: \mathrm{M} \rightarrow \Pi$.

The next step is to extend the equivalence relation $\sim$ to the entire set $\Psi$. Let $\alpha^{\prime}, \beta^{\prime} \in$ $\Psi \backslash \mathrm{M}$. We set $\alpha^{\prime} \sim \beta^{\prime}$ if and only if there exist $\operatorname{roots} \alpha, \beta \in \mathrm{M}$ and $\delta \in \Delta_{+}$such that $\alpha^{\prime} \in F(\alpha), \beta^{\prime} \in F(\beta), \alpha^{\prime}+\delta=\alpha$, and $\beta^{\prime}+\delta=\beta$.

Below we shall prove (see Proposition 9) that this relation is an equivalence relation on the set $\Psi \backslash \mathrm{M}$. We now point out two simple properties of this relation.

Lemma 20. Suppose that $\alpha^{\prime}, \beta^{\prime} \in \Psi \backslash \mathrm{M}, \alpha^{\prime} \sim \beta^{\prime}$, and roots $\alpha, \beta \in \mathrm{M}, \delta \in \Delta_{+}$are such that $\alpha^{\prime} \in F(\alpha), \beta^{\prime} \in F(\beta), \alpha^{\prime}+\delta=\alpha$, and $\beta^{\prime}+\delta=\beta$. Then $\alpha \sim \beta$.

Proof. Since both simple roots $\pi(\alpha), \pi(\beta)$ are contained in Supp $\delta$, they are contained in $\operatorname{Supp} \alpha \cap \operatorname{Supp} \beta$, but this is impossible for $\alpha \nsim \beta$ by condition (D).

Lemma 21. Suppose that $\alpha^{\prime}, \beta^{\prime} \in \Psi \backslash M, \alpha^{\prime} \neq \beta^{\prime}$, and $\alpha^{\prime} \sim \beta^{\prime}$. Then there exists exactly one root $\alpha \in \mathrm{M}$ with $\alpha^{\prime} \in F(\alpha)$.

Proof. We choose roots $\alpha, \beta \in \mathrm{M}, \delta \in \Delta_{+}$such that $\alpha^{\prime} \in F(\alpha), \beta^{\prime} \in F(\beta), \alpha^{\prime}+\delta=\alpha$, and $\beta^{\prime}+\delta=\beta$. Then it follows from the hypothesis that $\alpha \neq \beta$. Assume that there exists a root $\widetilde{\alpha} \in \mathrm{M}$ such that $\widetilde{\alpha} \neq \alpha$ and $\alpha^{\prime} \in F(\widetilde{\alpha})$. Then we have $\operatorname{Supp} \alpha^{\prime} \subset \operatorname{Supp} \widetilde{\alpha}$ and $\operatorname{Supp} \delta \subset \operatorname{Supp} \beta$, so that $\operatorname{Supp} \alpha \subset \operatorname{Supp} \widetilde{\alpha} \cup \operatorname{Supp} \beta$, which contradicts condition (C).

Proposition 9. The relation $\sim$ is an equivalence relation on $\Psi \backslash \mathrm{M}$.

Proof. Reflexivity and symmetry are obvious for $\sim$; therefore it suffices to prove transitivity. Suppose that $\alpha^{\prime}, \beta^{\prime}, \gamma^{\prime} \in \Psi \backslash \mathrm{M}$ are distinct roots and $\alpha^{\prime} \sim \beta^{\prime}, \alpha^{\prime} \sim \gamma^{\prime}$. We claim that $\beta^{\prime} \sim \gamma^{\prime}$. We have $\alpha=\alpha^{\prime}+\delta_{1}, \beta=\beta^{\prime}+\delta_{1}, \widetilde{\alpha}=\alpha^{\prime}+\delta_{2}, \gamma=\gamma^{\prime}+\delta_{2}$ for some $\operatorname{roots} \alpha, \beta, \widetilde{\alpha}, \gamma \in \mathrm{M}$ and $\delta_{1}, \delta_{2} \in \Delta_{+}$. By Lemma 21 we see that $\widetilde{\alpha}=\alpha$, so $\delta_{1}=\delta_{2}$ and $\beta^{\prime} \sim \gamma^{\prime}$.

Corollary 14. Suppose that $A \subset \Psi \backslash \mathrm{M}$ is an equivalence class containing more than one element. Then

(a) for every root $\alpha^{\prime} \in A$ there exists a unique root $\alpha \in \mathrm{M}$ such that $\alpha^{\prime} \in F(\alpha)$;

(b) the root $\delta=\alpha-\alpha^{\prime}$ is the same for all roots $\alpha^{\prime} \in A$;

(c) $A+\delta \subset \mathrm{M}$, and all roots in $A+\delta$ are pairwise equivalent. 
Proof. Part (a) follows from Lemma 21, part (b) from the proof of Proposition 9 part (c) from part (b) and Lemma 20.

Thus, we have an equivalence relation on each of the sets $M$ and $\Psi \backslash M$. We extend it to the whole set $\Psi$, setting $\alpha \nsim \beta$ for $\alpha \in \mathrm{M}, \beta \in \Psi \backslash \mathrm{M}$ or $\alpha \in \Psi \backslash \mathrm{M}, \beta \in \mathrm{M}$. Let $\Psi_{1}, \Psi_{2}, \ldots, \Psi_{K}$ be all the equivalence classes of the set $\Psi$ with respect to the relation $\sim$.

Proposition 10. (a) Suppose that for some $i, j \in\{1, \ldots, K\}, i \neq j$, roots $\alpha^{\prime} \in \Psi_{i}$ and $\delta \in \Delta_{+}$are such that $\alpha^{\prime}+\delta \in \Psi_{j}$. Then $\Psi_{i}+\delta \subset \Psi_{j}$.

(b) Suppose that $i \in\{1, \ldots, K\}$ and roots $\alpha^{\prime}, \alpha^{\prime \prime} \in \Psi_{i}$ are distinct. Then $\alpha^{\prime \prime}-\alpha^{\prime} \notin \Delta$.

(c) Suppose that $i, j \in\{1, \ldots, K\}, i \neq j$, and $\left|\Psi_{i}\right| \geq 2$. Then there exists at most one root $\delta \in \Delta_{+}$such that $\Psi_{i}+\delta \subset \Psi_{j}$.

Proof. (a) If $\left|\Psi_{i}\right|=1$, then there is nothing to prove. If $\Psi_{j} \subset \mathrm{M}$, then the required assertion follows from Corollary 14(c). Therefore in what follows we assume that $\left|\Psi_{i}\right| \geq 2$ and $\Psi_{j} \not \subset \mathrm{M}$. We set $\alpha^{\prime \prime}=\alpha^{\prime}+\delta, \alpha^{\prime \prime} \in \Psi_{j}$. Let $\alpha$ denote the unique root in $\mathrm{M}$ such that $\alpha^{\prime} \in F(\alpha)$ (uniqueness follows from Corollary 14(a)). Let $\widetilde{\alpha} \in \mathrm{M}$ be an arbitrary root such that $\alpha^{\prime \prime} \in F(\widetilde{\alpha})$. Then $\operatorname{Supp} \alpha^{\prime} \subset \operatorname{Supp} \widetilde{\alpha}$, whence by Lemma 18 we obtain $\alpha^{\prime} \in F(\widetilde{\alpha})$ and, in view of Corollary 14(a), $\widetilde{\alpha}=\alpha$. We set $\delta^{\prime}=\alpha-\alpha^{\prime} \in \Delta_{+}$and $\delta^{\prime \prime}=\alpha-\alpha^{\prime \prime} \in \Delta_{+}$. We now consider an arbitrary root $\beta^{\prime} \in \Psi_{i}$ and show that $\beta^{\prime \prime}=\beta^{\prime}+\delta \in \Psi_{j}$. We denote by $\beta$ the unique root in M such that $\beta^{\prime} \in F(\beta)$ (see Corollary 14(a)). From Corollary 14(b, c) we obtain $\beta=\beta^{\prime}+\delta^{\prime}$ and $\alpha \sim \beta$. Next, we have $\delta^{\prime}=\delta+\delta^{\prime \prime}$, whence $\left|\operatorname{Supp} \delta^{\prime}\right| \geq 2$. Furthermore, $\operatorname{Supp} \delta^{\prime} \subset \operatorname{Supp} \alpha \cap \operatorname{Supp} \beta$ and $\pi(\alpha), \pi(\beta) \in \operatorname{Supp} \delta^{\prime}$. Therefore possibility (E2) is realized for the roots $\alpha, \beta$. Hence the diagram $\Sigma(\operatorname{Supp} \alpha \cup \operatorname{Supp} \beta)$ has the form depicted in Figure 1 (for some $p, q, r \geq 1$ ), $\alpha=\alpha_{1}+\cdots+\alpha_{p}+\gamma_{0}+\gamma_{1}+\cdots+\gamma_{r}$, $\beta=\beta_{1}+\cdots+\beta_{q}+\gamma_{0}+\gamma_{1}+\cdots+\gamma_{r}$, and $\pi(\alpha)=\pi(\beta)=\gamma_{s}$ for some $s \in\{0,1, \ldots, r\}$. Furthermore, $\delta^{\prime}=\gamma_{t}+\gamma_{t+1}+\cdots+\gamma_{r}$, where $0 \leq t \leq s$, and $\delta^{\prime \prime}=\gamma_{u}+\gamma_{u+1}+\cdots+\gamma_{r}$, where $t<u \leq s$. It is easy to see that $\beta^{\prime \prime}=\beta-\delta^{\prime \prime}$ is a root contained in $F(\beta)$. Since $\beta^{\prime \prime}+\delta^{\prime \prime}=\beta, \alpha^{\prime \prime}+\delta^{\prime \prime}=\alpha$, and $\alpha \sim \beta$, it follows that $\alpha^{\prime \prime} \sim \beta^{\prime \prime}$ and $\beta^{\prime \prime} \in \Psi_{j}$, which completes the proof of part (a).

(b) Assume that $\delta_{0}=\alpha^{\prime \prime}-\alpha^{\prime} \in \Delta$. Without loss of generality we can also assume that $\delta_{0} \in \Delta_{+}$. By Corollary 14 there exist unique roots $\widetilde{\alpha}^{\prime}, \widetilde{\alpha}^{\prime \prime} \in \mathrm{M}, \delta \in \Delta_{+}$such that $\alpha^{\prime} \in F\left(\widetilde{\alpha}^{\prime}\right), \alpha^{\prime \prime} \in F\left(\widetilde{\alpha}^{\prime \prime}\right), \alpha^{\prime}+\delta=\widetilde{\alpha}^{\prime}, \alpha^{\prime \prime}+\delta=\widetilde{\alpha}^{\prime \prime}$. By Lemma 18 we have $\alpha^{\prime} \in F\left(\widetilde{\alpha}^{\prime \prime}\right)$, which contradicts Lemma 14(a).

(c) If $\Psi_{j} \subset \mathrm{M}$, then the assertion is valid by Corollary 14 In what follows we assume that $\Psi_{j} \not \subset \mathrm{M}$. Let $\delta$ be a root such that $\Psi_{i}+\delta \subset \Psi_{j}$. We claim that $\delta$ is uniquely determined by $i$ and $j$. By Corollary 14 there exist uniquely determined indices $k, l \in\{1, \ldots, K\}$ and roots $\delta_{i}, \delta_{j} \in \Delta_{+}$such that $\Psi_{k}, \Psi_{l} \subset \mathrm{M}, \Psi_{i}+\delta_{i} \subset \Psi_{k}$, and $\Psi_{j}+\delta_{j} \subset \Psi_{l}$. By part (a), for any root $\alpha \in \Psi_{i}$ we have $\alpha+\delta \in \Psi_{j}, \alpha+\delta_{i} \in \Psi_{k} \subset \mathrm{M}$, $\alpha+\delta+\delta_{j} \in \Psi_{l} \subset \mathrm{M}$, whence by Lemma 18 we obtain $\alpha \in F\left(\alpha+\delta+\delta_{j}\right)$. But then $\alpha+\delta_{i}=\alpha+\delta+\delta_{j}$ by Corollary 14(a). Consequently, $\delta=\delta_{i}-\delta_{j}$ and the root $\delta$ is uniquely determined by $i, j$.

4.3. In this subsection we construct an algebra $\mathfrak{n}$ which is going to be the tangent algebra of the unipotent radical of the desired solvable spherical subgroup.

We set $\mathfrak{u}_{i}=\bigoplus_{\alpha \in \Psi_{i}} \mathfrak{g}_{\alpha}$ for $i=1, \ldots, K$, and $\mathfrak{u}_{0}=\bigoplus_{\alpha \notin \Psi} \mathfrak{g}_{\alpha}$, so that $\mathfrak{u}=\mathfrak{u}_{0} \oplus \bigoplus_{i=1}^{K} \mathfrak{u}_{i}$. With each subspace $\mathfrak{u}_{i}, i=1, \ldots, K$, we associate a linear function $\xi_{i}: \mathfrak{u}_{i} \rightarrow \mathbb{C}$ as follows. First let $i$ be such that $\Psi_{i} \subset \mathrm{M}$. Then we take $\xi_{i}$ to be an arbitrary linear function whose restrictions to each of the root subspaces $\mathfrak{g}_{\alpha}, \alpha \in \Psi_{i}$ do not vanish. Next, for each $i$ such that $\Psi_{i} \not \subset \mathrm{M}$ and $\left|\Psi_{i}\right|=1$, for $\xi_{i}$ we take any nonzero linear function on the (one-dimensional) space $\mathfrak{u}_{i}$. Finally, if $i$ is such that $\Psi_{i} \not \subset \mathrm{M}$ and $\left|\Psi_{i}\right| \geq 2$, then we proceed as follows. By Corollary 14 there exist a unique $j \in\{1, \ldots, K\}$ and a unique root 
$\delta \in \Delta_{+}$such that $\Psi_{j} \subset \mathrm{M}$ and $\Psi_{i}+\delta \subset \Psi_{j}$. For every $x \in \mathfrak{u}_{i}$ we set $\xi_{i}(x)=\xi_{j}\left(\left[x, e_{\delta}\right]\right)$. Then $\xi_{i}$ is a linear function on $\mathfrak{u}_{i}$, and its restriction to $\mathfrak{g}_{\alpha}$ is nonzero for every $\alpha \in \Psi_{i}$.

Lemma 22. Suppose that $\Psi_{i}+\delta \subset \Psi_{j}$, where the indices $i, j \in\{1, \ldots, K\}$ are distinct, and $\delta \in \Delta_{+}$. Then there exists a constant $c_{i j} \neq 0$ such that $\xi_{i}(x)=c_{i j} \xi_{j}\left(\left[x, e_{\delta}\right]\right)$ for all $x \in \mathfrak{u}_{i}$.

Proof. If $\left|\Psi_{i}\right|=1$ or $\Psi_{j} \subset \mathrm{M}$, then the assertion follows from the definition of $\xi_{i}$. In what follows we assume that $\left|\Psi_{i}\right| \geq 2$ and $\Psi_{j} \not \subset \mathrm{M}$. From the proof of Proposition 10(c) there exist a unique $k \in\{1, \ldots, K\}$ and uniquely determined roots $\delta_{i}, \delta_{j} \in \Delta_{+}$such that $\Psi_{k} \subset \mathrm{M}, \Psi_{i}+\delta_{i} \subset \Psi_{k}, \Psi_{j}+\delta_{j} \subset \Psi_{k}$, and $\delta+\delta_{j}=\delta_{i}$. Let $x \in \mathfrak{u}_{i}$. Then by definition we have $\xi_{i}(x)=\xi_{k}\left(\left[x, e_{\delta_{i}}\right]\right)$ and $\xi_{j}\left(\left[x, e_{\delta}\right]\right)=\xi_{k}\left(\left[\left[x, e_{\delta}\right], e_{\delta_{j}}\right]\right)$. Applying the Jacobi identity we obtain

$$
\xi_{j}\left(\left[x, e_{\delta}\right]\right)=\xi_{k}\left(\left[x,\left[e_{\delta}, e_{\delta_{j}}\right]\right]\right)+\xi_{k}\left(\left[\left[x, e_{\delta_{j}}\right], e_{\delta}\right]\right) .
$$

Since $\left[e_{\delta}, e_{\delta_{j}}\right]=c e_{\delta_{i}}$ for some $c \neq 0$, we have $\xi_{j}\left(\left[x, e_{\delta}\right]\right)=c \xi_{k}\left(\left[x, e_{\delta_{i}}\right]\right)+\xi_{k}\left(\left[\left[x, e_{\delta_{j}}\right], e_{\delta}\right]\right)$.

To complete the proof it suffices to verify that $\left[x, e_{\delta_{j}}\right]=0$. This equality will hold if we prove that $\alpha+\delta_{j} \notin \Delta_{+}$for every $\alpha \in \Psi_{i}$. Assume that $\alpha+\delta_{j} \in \Delta_{+}$for some $\alpha \in \Psi_{i}$. Then the root $\alpha+\delta_{j}+\delta \in \Psi_{k}$ has the following representation as a sum of two positive roots: $\alpha+\delta_{j}+\delta=\left(\alpha+\delta_{j}\right)+\delta$. Since $\delta \notin \Psi$, we have $\alpha+\delta_{j} \in \Psi$. Furthermore, we have $\alpha+\delta \in \Psi$. Therefore, $\operatorname{Supp}\left(\alpha+\delta_{j}+\delta\right)=\operatorname{Supp}(\alpha+\delta) \cup \operatorname{Supp}\left(\alpha+\delta_{j}\right)$, which contradicts the conditions $\pi\left(\alpha+\delta_{j}+\delta\right) \notin \operatorname{Supp}(\alpha+\delta)$ and $\pi\left(\alpha+\delta_{j}+\delta\right) \notin \operatorname{Supp}\left(\alpha+\delta_{j}\right)$, which hold by Proposition $8(\mathrm{a})$.

For every $i=1, \ldots, K$ we set $\mathfrak{n}_{i}=\left\{x \in \mathfrak{u}_{i} \mid \xi_{i}(x)=0\right\}$. Obviously, $\mathfrak{n}_{i}=0$ for $\left|\Psi_{i}\right|=1$. We now consider the subspace $\mathfrak{n}=\mathfrak{u}_{0} \oplus \bigoplus_{i=1}^{K} \mathfrak{n}_{i} \subset \mathfrak{u}$.

Proposition 11. The subspace $\mathfrak{n}$ is a subalgebra of $\mathfrak{u}$.

Proof. Recall (see Lemma 19) that for every root $\alpha \in \Psi$ and every representation $\alpha=$ $\beta+\gamma$, where $\beta, \gamma \in \Delta_{+}$, exactly one of the roots $\beta, \gamma$ belongs to $\Psi$. Because of this, the proof reduces to verifying that $\left[\mathfrak{n}_{i}, \mathfrak{g}_{\delta}\right] \subset \mathfrak{n}$ for every $i=1, \ldots, K$ and $\delta \notin \Psi$. Let us verify this. If $\alpha+\delta \notin \Psi$ for all $\alpha \in \Psi_{i}$, then the inclusion $\left[\mathfrak{n}_{i}, \mathfrak{g}_{\delta}\right] \subset \mathfrak{n}$ holds automatically. If $\alpha+\delta \in \Psi_{j}$ for some $\alpha \in \Psi_{i}$ and some $j \in\{1, \ldots, K\}$ (then $i \neq j$ by Proposition 10(b)), then we obtain $\Psi_{i}+\delta \subset \Psi_{j}$ by Proposition [10(a). From the definition of the subspaces $\mathfrak{n}_{i}, \mathfrak{n}_{j}$ and Lemma 22 we have $\left[\mathfrak{n}_{i}, \mathfrak{g}_{\delta}\right] \subset \mathfrak{n}_{j} \subset \mathfrak{n}$.

Lemma 23. The torus $S$ normalizes the subalgebra $\mathfrak{n}$.

Proof. By condition (T), for any two roots $\alpha, \beta \in \mathrm{M}$ with $\alpha \sim \beta$ we have $\tau(\alpha)=\tau(\beta)$. Now let $\alpha^{\prime}, \beta^{\prime} \in \Psi \backslash \mathrm{M}$ and $\alpha^{\prime} \sim \beta^{\prime}$. Then the definition of the equivalence relation $\sim$ implies that there exist roots $\alpha, \beta \in \mathrm{M}$ and $\delta \in \Delta_{+}$such that $\alpha=\alpha^{\prime}+\delta$ and $\beta=\beta^{\prime}+\delta$. Then $\tau\left(\alpha^{\prime}\right)=\tau(\alpha)-\tau(\delta)=\tau(\beta)-\tau(\delta)=\tau\left(\beta^{\prime}\right)$. Hence for all $i=1, \ldots, K$ the subspace $\mathfrak{u}_{i}$ is $S$-invariant, and so is $\mathfrak{n}_{i}$. This proves the lemma.

4.4. This subsection is the final stage of the proof of Theorem 5 . We shall construct the subgroup $H \subset G$ and prove that it is spherical in $G$.

Let $N$ denote the unipotent subgroup of $G$ with tangent algebra $\mathfrak{n}$. We set $H=S N$. It follows from Lemma 23 that $H$ is a subgroup of $G$; moveover, $H=S \wedge N$ and $H$ is standardly embedded in $B$. For $i=1, \ldots, K$ we set $\varphi_{i}=\tau(\alpha) \in \mathfrak{X}(S)$, where $\alpha \in \Psi_{i}$ is an arbitrary root. It follows from the proof of Lemma 23 that the weight $\varphi_{i}$ is well defined.

Proposition 12. The subgroup $H$ is a connected solvable spherical subgroup of $G$ standardly embedded in $B$ such that $\Upsilon(H)=(S, \mathrm{M}, \pi, \sim)$. 
Before we prove this proposition, let us first prove several auxiliary lemmas.

Recall (see Proposition $8(\mathrm{~b})$ ) that the map $\pi: F(\alpha) \rightarrow \operatorname{Supp} \alpha$ is a bijection for every root $\alpha \in \Psi$.

Lemma 24. Suppose that $\alpha, \beta \in \mathrm{M}$ are distinct roots such that $\alpha \sim \beta$ and $\pi(\alpha)=\pi(\beta)$. Then:

(a) There exists a unique node $\eta(\alpha, \beta) \in \operatorname{Supp} \alpha \backslash \operatorname{Supp} \beta$ of the diagram $\Sigma(\Pi)$ connected by an edge to a node in $\operatorname{Supp} \alpha \cap \operatorname{Supp} \beta$.

(b) If a root $\alpha^{\prime} \in F(\alpha)$ is such that $\pi\left(\alpha^{\prime}\right)=\eta(\alpha, \beta)$, then there exists a root $\beta^{\prime} \in F(\beta)$ with $\alpha^{\prime} \sim \beta^{\prime}$.

Proof. The proof repeats word-for-word the proof of Lemma 13.

Lemma 25. Suppose that $\alpha, \beta, \gamma \in \mathrm{M}$ are pairwise distinct roots such that $\alpha \sim \beta \sim \gamma$ and $\pi(\alpha)=\pi(\beta)=\pi(\gamma)$. Then either $\operatorname{Supp} \alpha \cap \operatorname{Supp} \beta \subset \operatorname{Supp} \alpha \cap \operatorname{Supp} \gamma$ or $\operatorname{Supp} \alpha \cap$ $\operatorname{Supp} \gamma \subset \operatorname{Supp} \alpha \cap \operatorname{Supp} \beta$.

Proof. The proof is almost word-for-word the same as the proof of Lemma 14, with the difference that instead of referring to Proposition 5 we cite condition (E).

Lemma 26. Suppose that $\alpha, \beta \in \mathrm{M}, \alpha \neq \beta$, and $I=\operatorname{Supp} \alpha \cap \operatorname{Supp} \beta \neq \varnothing$. Let $\delta \in I$ be an arbitrary root and let $\alpha^{\prime} \in F(\alpha), \beta^{\prime} \in F(\beta)$ be such that $\pi\left(\alpha^{\prime}\right)=\pi\left(\beta^{\prime}\right)=\delta$.

(a) If either (D1) or (D2) is realized for $\alpha, \beta$, then $\alpha^{\prime}=\beta^{\prime}$.

(b) If either (E1) or (E2) is realized for $\alpha, \beta$, then $\alpha^{\prime} \sim \beta^{\prime}$.

In either case, $\alpha^{\prime} \sim \beta^{\prime}$.

Proof. By conditions (D) and (E), one of (D1), (D2), (E1), (E2) is realized for the roots $\alpha$ and $\beta$. Part (a) is obtained by a direct check. If one of (E1) or (E2) is realized, then we have $\alpha \sim \beta$. In the case of (E1), the set $I$ consists of the only root $\delta$. Then $\alpha^{\prime}=\alpha, \beta^{\prime}=\beta$, and $\alpha^{\prime} \sim \beta^{\prime}$. Finally, if (E2) is realized, then we have either $\alpha^{\prime}=\beta^{\prime}$ or $\alpha-\alpha^{\prime}=\beta-\beta^{\prime}$. In both cases, $\alpha^{\prime} \sim \beta^{\prime}$.

If $\Psi^{\prime} \subset \Psi$ is an arbitrary nonempty subset, then the restriction of the equivalence relation $\sim$ from $\Psi$ to $\Psi^{\prime}$ is well defined; therefore we can consider equivalence classes on $\Psi^{\prime}$.

Lemma 27. Suppose that $\mathrm{M}^{\prime} \subset \mathrm{M}$ is an arbitrary subset. Set $l=\left|\bigcup_{\delta \in \mathrm{M}^{\prime}} \operatorname{Supp} \delta\right|$, let $k$ be the number of equivalence classes in the set $\bigcup_{\delta \in \mathrm{M}^{\prime}} F(\delta)$, and let $m$ be the number of equivalence classes in the set $\mathrm{M}^{\prime}$. Then

(a) $\operatorname{dim}\left\langle\mu-\nu \mid \mu, \nu \in \mathrm{M}^{\prime}, \mu \sim \nu\right\rangle=\left|\mathrm{M}^{\prime}\right|-m$;

(b) $l \geq k+\left|\mathrm{M}^{\prime}\right|-m$.

Proof. We prove parts (a) and (b) by simultaneous induction on $\left|\mathrm{M}^{\prime}\right|$.

For $\left|\mathrm{M}^{\prime}\right|=1$ we have $\left|\mathrm{M}^{\prime}\right|=m$ and $\left\langle\mu-\nu \mid \mu, \nu \in \mathrm{M}^{\prime}, \mu \sim \nu\right\rangle=\{0\}$; therefore part (a) is true. Part (b) is also true by property (3) of roots in $\mathrm{M}$ (see $\S 4.1$ ).

We assume that parts (a) and (b) hold for all proper subsets of the set $\mathrm{M}^{\prime}$ and prove that they hold for $\mathrm{M}^{\prime}$. Suppose that $\mathrm{M}^{\prime}=\widetilde{\mathrm{M}}^{\prime} \cup\{\alpha\}$, where $\alpha \notin \widetilde{\mathrm{M}}^{\prime}$. Set $J=(\operatorname{Supp} \alpha) \backslash\left(\bigcup_{\delta \in \widetilde{\mathrm{M}}^{\prime}} \operatorname{Supp} \delta\right)$ and $\widetilde{l}=\left|\bigcup_{\delta \in \widetilde{\mathrm{M}}^{\prime}} \operatorname{Supp} \delta\right|$. Let $\widetilde{k}$ be the number of equivalence classes in the set $\bigcup_{\delta \in \widetilde{\mathrm{M}}^{\prime}} F(\delta)$, and let $\widetilde{m}$ be the number of equivalence classes in the set $\widetilde{\mathrm{M}}^{\prime}$. Clearly, $\left|\mathrm{M}^{\prime}\right|=\left|\mathrm{M}^{\prime}\right|+1$ and $l=\widetilde{l}+|J|$.

We further consider two cases.

Case 1. For every root $\delta \in \widetilde{\mathrm{M}}^{\prime}$ we have $\alpha \nsim \delta$. Then $m=\tilde{m}+1$ and the subspace $\left\langle\mu-\nu \mid \mu, \nu \in \mathrm{M}^{\prime}, \mu \sim \nu\right\rangle$ coincides with the subspace $\left\langle\mu-\nu \mid \mu, \nu \in \widetilde{\mathrm{M}}^{\prime}, \mu \sim \nu\right\rangle$, which has dimension $\left|\widetilde{M}^{\prime}\right|-\widetilde{m}=\left|\mathrm{M}^{\prime}\right|-m$ by the induction hypothesis. We have thus proved 
part (a). To prove part (b), in view of the induction hypothesis it suffices to verify that $|J| \geq k-\widetilde{k}$. This inequality holds by Lemma 26 .

Case 2. There exists a root $\alpha_{0} \in \widetilde{\mathrm{M}}^{\prime}$ such that $\alpha \sim \alpha_{0}$. Then we have $m=\widetilde{m}$. By condition (C) there exists a simple root $\rho \in \operatorname{Supp} \alpha$ such that $\rho \in J$; therefore $\alpha-\alpha_{0}$ is not contained in the subspace $\left\langle\mu-\nu \mid \mu, \nu \in \widetilde{\mathrm{M}}^{\prime}, \mu \sim \nu\right\rangle$. It is easy to see that the subspace $\left\langle\mu-\nu \mid \mu, \nu \in \mathrm{M}^{\prime}, \mu \sim \nu\right\rangle$ coincides with the subspace $\langle\mu-\nu| \mu, \nu \in \widetilde{\mathrm{M}}^{\prime}$, $\mu \sim \nu\rangle \oplus\left\langle\alpha-\alpha_{0}\right\rangle$; its dimension is equal to $\left|\widetilde{\mathrm{M}}^{\prime}\right|-\widetilde{m}+1=\left|\mathrm{M}^{\prime}\right|-m$ by the induction hypothesis. Part (a) is proved.

To prove part (b), by the induction hypothesis it suffices to verify that $|J| \geq k-\widetilde{k}+1$. We consider two subcases.

Subcase 2.1. $\pi(\alpha) \in J$. The required inequality holds by Lemma 26 and the condition $\alpha \sim \alpha_{0}$.

Subcase 2.2. $\pi(\alpha) \notin J$. In this situation there exists a root $\beta \in \widetilde{\mathrm{M}}^{\prime}$ such that $\pi(\alpha) \in \operatorname{Supp} \beta$. We assume without loss of generality that the set $\operatorname{Supp} \alpha \cap \operatorname{Supp} \beta$ is maximal with respect to inclusion. Let $\eta(\alpha, \beta)$ be the root from Lemma 24(a). Consider the root $\alpha^{\prime} \in F(\alpha)$ with $\pi\left(\alpha^{\prime}\right)=\eta(\alpha, \beta)$ and the root $\beta^{\prime} \in F(\beta)$ with $\beta^{\prime} \sim \alpha^{\prime}$ ( $\beta^{\prime}$ exists by Lemma 24(b)). We claim that $\eta(\alpha, \beta) \in J$. Assume the converse. Then there exist roots $\gamma \in \tilde{\mathrm{M}}^{\prime} \backslash\{\beta\}$ and $\gamma^{\prime} \in F(\gamma)$ such that $\pi\left(\gamma^{\prime}\right)=\pi\left(\alpha^{\prime}\right)$. If $\alpha^{\prime}=\gamma^{\prime}$, then we have Supp $\alpha^{\prime} \subset$ $\operatorname{Supp} \gamma$ and $\operatorname{Supp}\left(\alpha-\alpha^{\prime}\right)=\operatorname{Supp}\left(\beta-\beta^{\prime}\right) \subset \operatorname{Supp} \beta$, so that $\operatorname{Supp} \alpha \subset \operatorname{Supp} \beta \cup \operatorname{Supp} \gamma$, in contradiction to condition (C). Therefore, $\alpha^{\prime} \neq \gamma^{\prime}$, and by Lemma $26(\mathrm{a}, \mathrm{b})$ one of the possibilities (E1) or (E2) is realized for the roots $\alpha, \gamma$. In particular, $\alpha \sim \gamma$ and $\pi(\alpha)=\pi(\gamma)$, so by Lemma 25 we obtain $\operatorname{Supp} \alpha \cup \operatorname{Supp} \gamma \supsetneqq \operatorname{Supp} \alpha \cup \operatorname{Supp} \beta$, contradicting the choice of $\beta$. Thus, we have $\eta(\alpha, \beta) \in J$. Then the inequality $|J| \geq k-\widetilde{k}+1$ holds in view of Lemma 26 and the condition $\alpha^{\prime} \sim \beta^{\prime}$.

Part (b) is proved.

Proof of Proposition 12. We only need prove that $H$ is spherical; all the other conditions hold by construction. We consider the subspace $R=\left\langle\bigcup_{\delta \in \mathrm{M}} \operatorname{Supp} \delta\right\rangle \subset Q$ and denote its dimension by $l$. Let $m$ be the number of equivalence classes in M. By property (4) (see $\S 4.1$ ), the image of $R$ under the map $\tau$ is generated by the weights $\varphi_{1}, \ldots, \varphi_{K}$. By Lemma 27(a), the dimension of this image is equal to $l-(|\mathrm{M}|-m)$. Therefore, $K \geq l-(|\mathrm{M}|-m)$. On the other hand, $K \leq l-(|\mathrm{M}|-m)$ by Lemma 27(b). Consequently, $K=l-(m-n)$ and all the weights $\varphi_{1}, \ldots, \varphi_{K}$ are linearly independent. Furthermore, by construction the codimension of the subspace $\mathfrak{n}_{i}$ in the space $\mathfrak{u}_{i}$ is equal to 1 for all $i=1, \ldots, K$. Thus, condition (2) of Theorem 1 holds and $H$ is spherical in $G$.

The proof of Theorem 5 is complete.

Remark 4. As we see from the proof of Theorem 5. up to a conjugation by an element of $T$, the unipotent radical $N$ of a connected solvable spherical subgroup $H$ standardly embedded in $B$ can be uniquely reconstructed from the set $\Upsilon_{0}(H)=(\mathrm{M}, \pi, \sim)$ satisfying conditions (A), (D), (E), (C).

Remark 5. For every set $(\mathrm{M}, \pi, \sim)$ satisfying conditions $(\mathrm{A}),(\mathrm{D}),(\mathrm{E}),(\mathrm{C})$ there is at least one connected solvable spherical subgroup $H \subset G$ standardly embedded in $B$ such that $\Upsilon_{0}(H)=(\mathrm{M}, \pi, \sim)$. Namely, we can take $S$ to be the connected component of the identity of the subgroup of $T$ defined by the vanishing of all characters of the form $\alpha-\beta$, where $\alpha, \beta \in \mathrm{M}$ and $\alpha \sim \beta$. 


\section{$\S 5$. Classification of COnnected solvable spherical subgroups UP TO CONJUGACY}

Theorems 4 and 5 give a classification of connected solvable spherical subgroups of $G$ standardly embedded in $B$ up to a conjugation by an element of $T$. The goal of this section is to clarify the question of when two connected solvable spherical subgroups standardly embedded in $B$ are conjugate in $G$ and how the corresponding sets of combinatorial data are related in this case.

\subsection{The main result of this subsection is Proposition 13}

Let $H_{1}, H_{2} \subset G$ be two connected solvable subgroups standardly embedded in $B$. Let $N_{i}$ be the unipotent radical of $H_{i}$ and $S_{i} \subset T$ its maximal torus so that $H_{i}=S_{i} \wedge N_{i}$, $i=1,2$. We set $Z=Z_{G}\left(S_{1}\right)$. The group $Z$ is reductive and connected (as the centralizer of a torus in $G$ ), and its tangent algebra $\mathfrak{z}$ has the form $\mathfrak{z}=\mathfrak{t} \oplus \bigoplus_{\alpha \in \Delta: \tau(\alpha)=0} \mathfrak{g}_{\alpha}$, where $\tau: \mathfrak{X}(T) \rightarrow \mathfrak{X}\left(S_{1}\right)$ is the character restriction map.

Lemma 28. If $H_{2}=g H_{1} g^{-1}$ for some $g \in G$, then $g \in N_{2} \cdot N_{G}(T) \cdot Z$. In particular, $H_{2}=g_{0} H_{1} g_{0}^{-1}$ for some $g_{0} \in N_{G}(T) \cdot Z$.

Proof. We have $N_{2}=g N_{1} g^{-1}$. Next, there exists an element $u \in N_{2}$ such that $S_{2}=$ $g_{0} S_{1} g_{0}^{-1}$ for $g_{0}=u g$. By the Bruhat decomposition of $G$ we have $g_{0}=u_{1} \sigma u_{2}$, where $u_{1}, u_{2} \in U, \sigma \in N_{G}(T)$.

We consider an arbitrary element $s_{1} \in S_{1}$ and set $s_{2}=g_{0} s_{1} g_{0}^{-1} \in S_{2}$. Then we have $u_{1}^{-1} s_{2} u_{1} \sigma=\sigma u_{2} s_{1} u_{2}^{-1}$, which can be rewritten in the form $s_{2} v_{1} \sigma=\sigma s_{1} v_{2}$, where $v_{1}=s_{2}^{-1} u_{1}^{-1} s_{2} u_{1} \in U$ and $v_{2}=s_{1}^{-1} u_{2} s_{1} u_{2}^{-1} \in U$. Hence, $\sigma v_{2} \sigma^{-1}=\left(\sigma s_{1}^{-1} \sigma^{-1}\right) s_{2} v_{1} \in$ $B$. Since $v_{2}$ is a unipotent element, we have $\sigma v_{2} \sigma^{-1} \in U$. Therefore, $\sigma v_{2} \sigma^{-1} v_{1}^{-1}=$ $\left(\sigma s_{1}^{-1} \sigma^{-1}\right) s_{2} \in U \cap T=\{e\}$, so $s_{2}=\sigma s_{1} \sigma^{-1}$ and $v_{2}=\sigma v_{1} \sigma^{-1}$. Thus, $s_{1}=\sigma^{-1} g_{0} s_{1} g_{0}^{-1} \sigma$ for any element $s_{1} \in S_{1}$; therefore, $\sigma^{-1} g_{0} \in Z$ and $g_{0} \in N_{G}(T) \cdot Z$.

Proposition 13. If both of the subgroups $H_{1}, H_{2}$ are spherical in $G$ and $H_{2}=g H_{1} g^{-1}$ for some $g \in G$, then $g \in N_{2} \cdot N_{G}(T) \cdot N_{1}$. In particular, $H_{2}=\sigma H_{1} \sigma^{-1}$ for some $\sigma \in N_{G}(T)$.

Proof. By Lemma 28 we can assume that $g=u \sigma z$, where $u \in N_{2}, \sigma_{0} \in N_{G}(T), z \in Z$. Consider the subalgebra $\mathfrak{u}_{0}=\bigoplus_{\alpha \in \Delta_{+}: \tau(\alpha)=0} \mathfrak{g}_{\alpha}$ of the Lie algebra $\mathfrak{z}$. It is the tangent algebra of a maximal unipotent subgroup $U_{0}$ of $Z$. Furthermore, $\mathfrak{u}_{0} \subset \mathfrak{h}_{1}$. Since $\operatorname{Ad}(g) \mathfrak{u}_{0} \subset \mathfrak{u}$, it follows that $\operatorname{Ad}(z) \mathfrak{u}_{0} \subset \operatorname{Ad}\left(\sigma_{0}^{-1}\right) \mathfrak{u}$. Hence the projection of the algebra $\operatorname{Ad}(z) \mathfrak{u}_{0}$ to the subspace $\mathfrak{t} \subset \mathfrak{z}$ is zero and for every root $\alpha \in \Delta$ the projection of $\operatorname{Ad}(z) \mathfrak{u}_{0}$ to one of the spaces $\mathfrak{g}_{\alpha}$ or $\mathfrak{g}_{-\alpha}$ is zero. From dimension considerations we obtain that $\operatorname{Ad}(z) \mathfrak{u}_{0}$ is a regular subalgebra of $\mathfrak{z}$ (that is, normalized by $T$ ), and the subalgebra $\mathfrak{t} \oplus \operatorname{Ad}(z) \mathfrak{u}_{0}$ is a Borel subalgebra of $\mathfrak{z}$ containing the Cartan subalgebra $\mathfrak{t}$. Therefore there exists an element $\sigma_{1} \in N_{Z}(T) \subset N_{G}(T)$ such that $\operatorname{Ad}\left(\sigma_{1}\right)\left(\mathfrak{t} \oplus \operatorname{Ad}(z) \mathfrak{u}_{0}\right)=\mathfrak{t} \oplus \mathfrak{u}_{0}$ and $\operatorname{Ad}\left(\sigma_{1} z\right) \mathfrak{u}_{0}=\mathfrak{u}_{0}$. Thus, $z \in N_{G}(T) \cdot N_{Z}\left(U_{0}\right)$. Since $N_{Z}\left(U_{0}\right)=T \curlywedge U_{0}$ and $U_{0} \subset N_{1}$, it follows that $z \in N_{G}(T) \cdot N_{1}$. Hence we finally obtain $g \in N_{2} \cdot N_{G}(T) \cdot N_{1}$.

5.2. In this subsection we introduce the notion of an elementary transformation and prove the main theorem of the section (Theorem 6). Throughout this subsection we use the notation from $\S 2.2$

Let $H \subset G$ be a connected solvable spherical subgroup standardly embedded in $B$.

Definition 7. An active root $\alpha$ is called regular if the set $\Psi_{i}$ containing $\alpha$ consists of a single element, that is, $\Psi_{i}=\{\alpha\}$. 
It is easy to see that an active root $\alpha$ is regular if and only if the projection of the subspace $\mathfrak{n} \subset \mathfrak{u}$ to $\mathfrak{g}_{\alpha}$ is zero. It is also clear that the subgroup $H$ is regular (that is, is normalized by $T$ ) if and only if all its active roots are regular.

We denote by $\Psi^{\text {reg }}(H)$ the set of regular active roots of $H$. We also set $\Omega(H)=$ $\Delta_{+} \backslash \Psi^{\mathrm{reg}}(H)$. Clearly, $\alpha \in \Omega(H)$ if and only if the projection of the space $\mathfrak{n}$ to $\mathfrak{g}_{\alpha}$ is nonzero.

Definition 8. Let $\alpha \in \Psi^{\mathrm{reg}}(H) \cap \Pi$. An elementary transformation with centre $\alpha$ (or simply an elementary transformation) is the transition from the subgroup $H$ to the subgroup $\sigma_{\alpha} H \sigma_{\alpha}^{-1}$, where $\sigma_{\alpha} \in N_{G}(T)$ is some representative of the reflection $r_{\alpha}$.

Since $\alpha$ is the only positive root which is taken to a negative root under the simple reflection $r_{\alpha}$, the subgroup $\sigma_{\alpha} H \sigma_{\alpha}^{-1}$ is also standardly embedded in $B$.

Let $C_{0} \subset Q$ be the dominant Weyl chamber, that is, $C_{0}=\{x \in Q \mid(x, \alpha) \geq 0$ for all $\alpha \in \Pi\}$. For every Weyl chamber $C \subset Q$, let $P(C)$ denote the set of those roots in $\Delta$ that are positive with respect to $C$. Clearly, $P\left(C_{0}\right)=\Delta_{+}$.

Let us study the following question. Suppose that we are given a connected solvable spherical subgroup $H \subset G$ standardly embedded in $B$. Let us find all subgroups conjugate to $H$ that are also standardly embedded in $B$. Let $H^{\prime}$ be such a subgroup. By Proposition 13, $H^{\prime}=\sigma H \sigma^{-1}$ for some $\sigma \in N_{G}(T)$. Let $w$ be the image of the element $\sigma$ in the group $W$. Then $w \Omega(H) \subset \Delta_{+}=P\left(C_{0}\right)$, so $\Omega(H) \subset P\left(w^{-1} C_{0}\right)$. Conversely, suppose that $C$ is a Weyl chamber such that $\Omega(H) \subset P(C)$. Let $w_{C}$ denote the (unique) element of $W$ such that $C=w_{C}^{-1} C_{0}$. Then, obviously, the subgroup $H^{\prime}=\bar{w}_{C} H \bar{w}_{C}^{-1}$ is standardly embedded in $B$.

Lemma 29. A Weyl chamber $C$ satisfies the condition $\Omega(H) \subset P(C)$ if and only if it is contained in the cone $X(H)=\{x \mid(x, \alpha) \geq 0$ for all $\alpha \in \Omega(H)\} \subset Q$.

Proof. This follows from the fact that for a root $\alpha$ the condition $\alpha \in P(C)$ is equivalent to the condition that $(\alpha, x) \geq 0$ for all $x \in C$.

Let $H, H^{\prime}, \sigma, w$ be as above. Then $\Omega(H) \subset P\left(w^{-1} C_{0}\right)$ and, by Lemma 29, $w^{-1} C_{0} \subset$ $X(H)$. Being the intersection of several half-spaces, the cone $X(H)$ is convex. Clearly, it is a union of several Weyl chambers. Therefore there exist Weyl chambers $C_{1}, C_{2}, \ldots$, $C_{n-1}$ contained in $X(H)$ such that in the sequence $C_{0}, C_{1}, \ldots, C_{n-1}, C_{n}=w^{-1} C_{0}$ any two neighbouring Weyl chambers have a common hyperface. For $i=1, \ldots, n$, let $w_{i}$ denote the reflection with respect to the common hyperface of the chambers $C_{i-1}$ and $C_{i}, w_{i} \in W, w_{i}^{2}=e$. Then for $i=1, \ldots, n$ we have $C_{i}=w_{i} w_{i-1} \ldots w_{1} C_{0}$. Next, for every $i=1, \ldots, n$ there exists a simple reflection $r_{i}$ satisfying the condition $w_{i}=$ $\left(w_{i-1} w_{i-2} \ldots w_{1}\right) r_{i}\left(w_{i-1} w_{i-2} \ldots w_{1}\right)^{-1}$. Denote by $\alpha_{i}$ the simple root corresponding to the reflection $r_{i}$. Then $C_{i}=r_{1} \ldots r_{i-1} r_{i} C_{0}=\left(r_{i} r_{i-1} \ldots r_{1}\right)^{-1} C_{0}, i=1, \ldots, n$. Recall that $C_{i} \subset X(H)$ for all $i=1, \ldots, n$; therefore, in view of Lemma 29, the subgroup $H_{i}=\left(\bar{r}_{i} \bar{r}_{i-1} \ldots \bar{r}_{1}\right) H\left(\bar{r}_{i} \bar{r}_{i-1} \ldots \bar{r}_{1}\right)^{-1}$ is standardly embedded in $B$ for $i=1, \ldots, n$. Hence, $H_{i}=\bar{r}_{i} H_{i-1} \bar{r}_{i}^{-1}$ (we put $H_{0}=H$ ), $\alpha_{i}$ is an active root with respect to $H_{i-1}$, and the transition $H_{i-1} \mapsto H_{i}$ is an elementary transformation with centre $\alpha_{i}$. Clearly, $H^{\prime}=t H_{n} t^{-1}$ for some element $t \in T$. Then the chain of elementary transformations

$$
H \mapsto H_{1} \mapsto \cdots \mapsto H_{n-1} \mapsto\left(t \bar{r}_{\alpha_{n}}\right) H_{n-1}\left(t \bar{r}_{\alpha_{n}}\right)^{-1}=H^{\prime}
$$

takes $H$ to $H^{\prime}$. Thus we have proved the following theorem.

Theorem 6. Two connected solvable spherical subgroups $H, H^{\prime} \subset G$ standardly embedded in $B$ are conjugate in $G$ if and only if $H^{\prime}$ can be obtained from $H$ by applying a suitable chain of elementary transformations. 
Theorems 4, 5] and 6 provide a complete classification of connected solvable spherical subgroups of $G$ up to conjugacy.

5.3. We now explore how the set of combinatorial data of a connected solvable spherical subgroup changes under an elementary transformation. Consider two connected solvable spherical subgroups $H$ and $H^{\prime}$ standardly embedded in $B$ that can be obtained from each other by an elementary transformation with centre $\alpha$, where $\alpha$ is a regular active simple root of $H$ (and of $\left.H^{\prime}\right)$. Let $\Upsilon(H)=(S, \mathrm{M}, \pi, \sim)$ and $\Upsilon\left(H^{\prime}\right)=\left(S^{\prime}, \mathrm{M}^{\prime}, \pi^{\prime}, \sim^{\prime}\right)$. We have $H^{\prime}=\sigma_{\alpha} H \sigma_{\alpha}^{-1}$ for some representative $\sigma_{\alpha} \in N_{G}(T)$ of the reflection $r_{\alpha}$, whence we obviously obtain $S^{\prime}=\sigma_{\alpha} S \sigma_{\alpha}^{-1}$.

Lemma 30. The following hold:

(a) $\Psi\left(H^{\prime}\right)=r_{\alpha}(\Psi(H) \backslash\{\alpha\}) \cup\{\alpha\}$;

(b) $\pi^{\prime}\left(r_{\alpha}(\beta)\right)=\pi(\beta)$ for $\beta \in \Psi(H) \backslash\{\alpha\}$;

(c) $\mathrm{M}^{\prime} \backslash\{\alpha\}=r_{\alpha}(\mathrm{M} \backslash\{\alpha\})$.

Proof. Part (a) is obvious. Let us prove (b). Suppose that $\beta \in \Psi(H) \backslash\{\alpha\}$. First we observe that $\pi(\beta) \neq \alpha$, so $\pi(\beta) \in \operatorname{Supp} r_{\alpha}(\beta)$. Let $r_{\alpha}(\beta)=\beta_{1}+\beta_{2}$ be an arbitrary decomposition of the root $r_{\alpha}(\beta) \in \Psi\left(H^{\prime}\right)$ into a sum of two positive roots such that $\beta_{1} \in \Psi\left(H^{\prime}\right)$. It suffices to show that $\pi(\beta) \notin \operatorname{Supp} \beta_{1}$. This holds if $\beta_{1}=\alpha$; therefore in what follows we assume that $\beta_{1} \neq \alpha$. Furthermore, we have $\beta_{2} \neq \alpha$ since $\beta_{2} \notin$ $\Psi\left(H^{\prime}\right)$. Therefore on the right-hand side of the expression $\beta=r_{\alpha}\left(\beta_{1}\right)+r_{\alpha}\left(\beta_{2}\right)$ both roots are positive and $r_{\alpha}\left(\beta_{1}\right) \in \Psi(H)$. Hence $\pi(\beta) \notin \operatorname{Supp} r_{\alpha}\left(\beta_{1}\right)$. Moreover, $\pi(\beta) \notin$ $\operatorname{Supp} r_{\alpha}\left(\beta_{1}\right) \cup\{\alpha\}$. But $\operatorname{Supp} \beta_{1} \subset \operatorname{Supp} r_{\alpha}\left(\beta_{1}\right) \cup\{\alpha\}$, and so $\pi(\beta) \notin \operatorname{Supp} \beta_{1}$, as required.

We now prove (c). Suppose that $\beta \in \mathrm{M} \backslash\{\alpha\}$. Assume that the root $r_{\alpha}(\beta) \in$ $\Psi\left(H^{\prime}\right) \backslash\{\alpha\}$ is not a maximal active root with respect to $H^{\prime}$. In this case there exist roots $\delta \in \Psi\left(H^{\prime}\right) \backslash\{\alpha\}$ and $\gamma \in \Delta_{+} \backslash \Psi\left(H^{\prime}\right)$ such that $r_{\alpha}(\beta)+\gamma=\delta$. In particular, $\gamma \neq \alpha$, whence $r_{\alpha}(\gamma) \in \Delta_{+}$. The active root $r_{\alpha}(\delta)$ has the representation $r_{\alpha}(\delta)=\beta+r_{\alpha}(\gamma)$ as a sum of two positive roots, which contradicts the maximality of the active root $\beta$. Thus, $r_{\alpha}(\mathrm{M} \backslash\{\alpha\}) \subset \mathrm{M}^{\prime} \backslash\{\alpha\}$. Similarly, $r_{\alpha}\left(\mathrm{M}^{\prime} \backslash\{\alpha\}\right) \subset \mathrm{M} \backslash\{\alpha\}$, giving the required result.

The following proposition is a consequence of the preceding arguments and Lemma 30.

Proposition 14. The sets of combinatorial data $\Upsilon(H)$ and $\Upsilon\left(H^{\prime}\right)$ are related as follows:

(1) $S^{\prime}=\sigma_{\alpha} S \sigma_{\alpha}^{-1}$

(2.1) if $\alpha \in \operatorname{Supp} \delta$ for some root $\delta \in r_{\alpha}(\mathrm{M} \backslash\{\alpha\})$, then

(a) $\mathrm{M}^{\prime}=r_{\alpha}(\mathrm{M} \backslash\{\alpha\})$;

(b) $\pi^{\prime}(\beta)=\pi\left(r_{\alpha}(\beta)\right)$ for every $\beta \in \mathrm{M}^{\prime}$;

(c) for all $\beta, \gamma \in \mathrm{M}^{\prime}$ the relation $\beta \sim^{\prime} \gamma$ holds if and only if $r_{\alpha}(\beta) \sim r_{\alpha}(\gamma)$;

(2.2) if $\alpha \notin \operatorname{Supp} \delta$ for all $\delta \in r_{\alpha}(\mathrm{M} \backslash\{\alpha\})$, then

(a) $\mathrm{M}^{\prime}=r_{\alpha}(\mathrm{M} \backslash\{\alpha\}) \cup\{\alpha\}$;

(b) $\pi^{\prime}(\beta)=\pi\left(r_{\alpha}(\beta)\right)$ for every $\beta \in \mathrm{M}^{\prime} \backslash\{\alpha\}, \pi^{\prime}(\alpha)=\alpha$;

(c) for all $\beta, \gamma \in \mathrm{M}^{\prime} \backslash\{\alpha\}$ the relation $\beta \sim^{\prime} \gamma$ holds if and only if $r_{\alpha}(\beta) \sim r_{\alpha}(\gamma)$; for every $\beta \in \mathrm{M}^{\prime} \backslash\{\alpha\}$ the relation $\beta \chi^{\prime} \alpha$ holds.

5.4. Let $H \subset G$ be a connected solvable spherical subgroup standardly embedded in $B$, and let $\Upsilon(H)=(S, \mathrm{M}, \pi, \sim)$. In this subsection we shall find out how to determine which simple roots are regular active roots of $H$ given the set $\Upsilon_{0}(H)=(\mathrm{M}, \pi, \sim)$. Here we use the same notation as in $\S 2.2$.

Proposition 15. A root $\alpha \in \Pi$ is a regular active root of $H$ in exactly one of the following two cases:

(1) $\alpha \in \mathrm{M}$ and $\beta \nsim \alpha$ for all $\beta \in \mathrm{M} \backslash\{\alpha\}$; 
(2) $\alpha \notin \mathrm{M}$ and there exists a root $\alpha^{\prime} \in \mathrm{M}$ such that
(a) the root $\alpha$ is terminal with respect to Supp $\alpha^{\prime}$;
(b) $\alpha \neq \pi\left(\alpha^{\prime}\right)$;
(c) $\operatorname{Supp} \alpha^{\prime} \backslash\{\alpha\} \not \subset \operatorname{Supp} \beta$ for all roots $\beta \in \mathrm{M} \backslash\left\{\alpha^{\prime}\right\}$.

Proof. Let $\alpha \in \Pi$ be a regular active root of $H$. If $\alpha \in \mathrm{M}$, then, obviously, condition (1) holds. Now suppose $\alpha \notin \mathrm{M}$. Then $\alpha \in F\left(\alpha^{\prime}\right)$ for some root $\alpha^{\prime} \in \mathrm{M}$, and $\alpha \neq \pi\left(\alpha^{\prime}\right)$. By Corollary 7 the root $\alpha$ is terminal with respect to $\operatorname{Supp} \alpha^{\prime}$. Assume that $\operatorname{Supp} \alpha^{\prime} \backslash\{\alpha\} \subset \operatorname{Supp} \beta$ for some root $\beta \in \mathrm{M} \backslash\left\{\alpha^{\prime}\right\}$. Then in view of the condition $\pi\left(\alpha^{\prime}\right) \neq \alpha$ and Propositions 4 , 5 we obtain that $\alpha^{\prime} \sim \beta$ and exactly one of the possibilities (E1) or (E2) is realized for $\alpha^{\prime}, \beta$. If (E1) is realized, then $\operatorname{Supp} \alpha^{\prime}=\left\{\alpha, \pi\left(\alpha^{\prime}\right)\right\}$. Since $\alpha^{\prime}-\pi\left(\alpha^{\prime}\right) \in F\left(\alpha^{\prime}\right)$ and $\pi\left(\alpha^{\prime}\right) \notin \operatorname{Supp}\left(\alpha^{\prime}-\pi\left(\alpha^{\prime}\right)\right)$, we have $\alpha^{\prime}-\pi\left(\alpha^{\prime}\right)=\alpha$. Hence we obtain that $\tau(\alpha)=\tau\left(\beta-\pi\left(\alpha^{\prime}\right)\right)$ and the root $\alpha$ is not regular. If (E2) is realized, then the root system $\Delta_{+} \cap\left\langle\operatorname{Supp} \alpha^{\prime} \cup \operatorname{Supp} \beta\right\rangle$ has type $\mathrm{D}$ or $\mathrm{E}$, whence $\alpha^{\prime}-\alpha \in \Delta_{+}$and $\beta-\left(\alpha^{\prime}-\alpha\right) \in F(\beta)$. Then $\tau(\alpha)=\tau\left(\beta-\left(\alpha^{\prime}-\alpha\right)\right)$ and the root $\alpha$ is not regular. The contradiction thus obtained proves that condition (2) holds.

Conversely, if condition (1) holds, then, obviously, $\alpha$ is a regular active root. Now suppose that condition (2) holds. By Corollary 8 we obtain that $\alpha \in \Psi$. Assume that $\alpha$ is not a regular active root. Then $\tau(\alpha)=\tau(\gamma)$ for some root $\gamma \in \Psi \backslash\{\alpha\}$. By Proposition 1 we have $\beta=\gamma+\left(\alpha^{\prime}-\alpha\right) \in \mathrm{M}$, whence Supp $\alpha^{\prime} \backslash\{\alpha\}=\operatorname{Supp} \alpha^{\prime} \cap \operatorname{Supp} \beta$, which contradicts condition (c).

Remark 6. Propositions 14 and 15, together with Remark 5] enable one to make the notion of an elementary transformation for sets $(\mathrm{M}, \pi, \sim)$ satisfying conditions (A), (D), $(\mathrm{E})$, and (C) well defined.

5.5. In this subsection we apply the theory presented above to one important class of connected solvable spherical subgroups. Namely, we obtain a classification, up to conjugacy, of all connected solvable spherical subgroups of $G$ having finite index in their normalizers. Following Vinberg (see [12, §1.3.4]), we say that connected spherical subgroups $H \subset G$ such that $H=N_{G}(H)^{0}$ are saturated ${ }^{2}$ Besides, we obtain a classification, up to conjugacy, of all unipotent subgroups of $G$ that are the unipotent radicals of connected solvable spherical subgroups of $G$.

Lemma 31. Let $H \subset G$ be a connected solvable spherical subgroup standardly embedded in $B$, and let $N=H \cap U$. Then $N_{G}(H)^{0}=\widehat{S} \wedge N$, where $\widehat{S}=\left(N_{G}(N) \cap T\right)^{0}$.

Proof. Let $S=H \cap T$. Let $\mathfrak{n}(\mathfrak{h})$ be the normalizer of the algebra $\mathfrak{h}$ in $\mathfrak{g}$. It suffices to prove that $\mathfrak{n}(\mathfrak{h})=\widehat{\mathfrak{s}}+\mathfrak{n}$. It is easy to see that $\mathfrak{n}(\mathfrak{h}) \subset \mathfrak{z}+\mathfrak{h}$, where $\mathfrak{z}$ is the centralizer of the algebra $\mathfrak{s}$ in $\mathfrak{g}$; it is the tangent algebra of the reductive group $Z_{G}(S)$. Let $\mathfrak{u}_{0}=\mathfrak{z} \cap \mathfrak{u}$. By the sphericity of $H$ we have $\mathfrak{u}_{0} \subset \mathfrak{h}$, whence $\mathfrak{z} \cap \mathfrak{h}=\mathfrak{s}+\mathfrak{u}_{0}$. Clearly, $\mathfrak{u}_{0}$ is a maximal nilpotent subalgebra of $\mathfrak{z}$; therefore its normalizer in $\mathfrak{z}$ coincides with the algebra $\mathfrak{t}+\mathfrak{u}_{0}$. Hence, $\mathfrak{n}(\mathfrak{h}) \subset \mathfrak{t}+\mathfrak{h}$ and therefore $\mathfrak{n}(\mathfrak{h})=\widehat{\mathfrak{s}}+\mathfrak{n}$.

Corollary 15. Every saturated solvable spherical subgroup $H \subset G$ standardly embedded in $B$ has the form $H=S \wedge N$, where $N=H \cap U$ and $S=\left(N_{G}(N) \cap T\right)^{0}$. In particular, the torus $S$ is the connected component of the identity of the subgroup of $T$ defined by the vanishing of all characters of the form $\alpha-\beta$, where $\alpha, \beta$ run over all roots in the set $\mathrm{M}(H)$ with $\alpha \sim \beta$.

Corollary 16. For every connected solvable spherical subgroup $H \subset G$, the subgroup $N_{G}(H)^{0}$ is a saturated solvable spherical subgroup of $G$.

\footnotetext{
${ }^{2}$ Another possible term is sober.
} 
Lemma 32. Up to conjugacy, every saturated solvable spherical subgroup is uniquely determined by its unipotent radical.

Proof. By Lemma 31, a maximal torus of a saturated solvable spherical subgroup $H \subset G$ with unipotent radical $N$ is a maximal torus in the group $N_{G}(N)$. This implies the assertion of the lemma, since all maximal tori in $N_{G}(N)$ are conjugate.

Corollary 17. Let $H \subset G$ be a saturated solvable spherical subgroup and $N$ its unipotent radical. Then the map $H \mapsto N$ defines a bijection between the conjugacy classes in $G$ of saturated solvable spherical subgroups and the conjugacy classes in $G$ of unipotent radicals of connected solvable spherical subgroups.

Proof. The injectivity of the map follows from Lemma 32 , and the surjectivity follows from Lemma 31 and Corollary 16.

We denote by $\widetilde{\Upsilon}_{0}$ the set of all triples $(\mathrm{M}, \pi, \sim)$, where $\mathrm{M} \subset \Delta_{+}$is a subset, $\pi$ : $\mathrm{M} \rightarrow \Pi$ is a map, and $\sim$ is an equivalence relation on $\mathrm{M}$ satisfying conditions (A), (D), (E), and (C). Recall (see Remarks 4 and 5) that to every triple $(\mathrm{M}, \pi, \sim) \in \widetilde{\Upsilon}_{0}$ there corresponds a subgroup $N=N(\mathrm{M}, \pi, \sim) \subset U$, determined uniquely up to a conjugation by an element of $T$, and this subgroup is the unipotent radical of a connected solvable spherical subgroup of $G$ standardly embedded in $B$.

Proposition 16. (a) The map $H \mapsto \Upsilon_{0}(H)$ is a bijection between the set of saturated solvable spherical subgroups of $G$ standardly embedded in $B$, up to a conjugation by an element of $T$, and the set $\widetilde{\Upsilon}_{0}$. Two saturated solvable spherical subgroups $H_{1}, H_{2} \subset$ $G$ standardly embedded in $B$ are conjugate in $G$ if and only if there exists a chain of elementary transformations taking the set $\Upsilon_{0}\left(H_{1}\right)$ to the set $\Upsilon_{0}\left(H_{2}\right)$.

(b) The map $(\mathrm{M}, \pi, \sim) \mapsto N(\mathrm{M}, \pi, \sim)$ is a bijection between the set $\widetilde{\Upsilon}_{0}$ and the set of unipotent radicals of connected solvable spherical subgroups in $G$ standardly embedded in $B$, up to a conjugation by an element of $T$. Two subgroups $N(\mathrm{M}, \pi, \sim), N\left(\mathrm{M}^{\prime}, \pi^{\prime}, \sim^{\prime}\right)$ are conjugate in $G$ if and only if there exists a chain of elementary transformations taking the set $(\mathrm{M}, \pi, \sim)$ to the set $\left(\mathrm{M}^{\prime}, \pi^{\prime}, \sim^{\prime}\right)$.

In particular, the set of conjugacy classes in $G$ of saturated solvable spherical subgroups, as well as the set of conjugacy classes in $G$ of unipotent radicals of connected solvable spherical subgroups, is finite.

Proof. (a) The injectivity of the map follows from Remark 4, Corollary 15] and Theorem 4. Let us prove the surjectivity. Let $(\mathrm{M}, \pi, \sim) \in \widetilde{\Upsilon}_{0}$. Consider the subtorus $S \subset T$ that is the connected component of the identity of the subgroup of $T$ defined by the vanishing of all characters of the form $\alpha-\beta$, where $\alpha, \beta$ run over all roots in $\mathrm{M}$ with $\alpha \sim \beta$. Then $S$ satisfies condition (T) (and it is the largest subtorus of $T$ satisfying this condition), whence by Theorem [5, up to a conjugation by an element of $T$, there exists a unique connected solvable spherical subgroup $H$ standardly embedded in $B$ such that $\Upsilon(H)=(S, \mathrm{M}, \pi, \sim)$. Let $N \subset U$ be the unipotent radical of $H$. Then, obviously, $S=\left(N_{G}(N) \cap T\right)^{0}$ and by Corollary 15 the subgroup $H$ is saturated. The second assertion of part (a) follows from the first one and Theorem 6 .

(b) Let $H \subset G$ be a saturated solvable spherical subgroup standardly embedded in $B$, and let $\Upsilon_{0}(H)=(\mathrm{M}, \pi, \sim)$. Then, up to a conjugation by an element of $T$, the subgroup $N=N(\mathrm{M}, \pi, \sim)$ is the unipotent radical of $H$. The required assertion now follows from part (a) and Corollary 17 


\section{$\S 6$. Simplification of the Set of COMbinatorial DATA CORRESPONDING TO A CONNECTED SOLVABLE SPHERICAL SUBGROUP}

This section is devoted to problems related to "simplification" of the set of combinatorial data $\Upsilon(H)$ corresponding to a connected solvable spherical subgroup $H \subset G$ standardly embedded in $B$.

6.1. In this subsection we show that every conjugacy class in $G$ of connected solvable spherical subgroups contains a subgroup $H$ standardly embedded in $B$ such that the set $\Upsilon(H)$ satisfies stronger conditions than those appearing in Theorem 4 . Thus the set $\Upsilon(H)$ is in a sense simpler than the set of the general form.

Until the end of this subsection $H$ stands for a connected solvable spherical subgroup of $G$ standardly embedded in $B$.

Definition 9. An active root $\alpha$ is called typical if it is equal to the sum of all simple roots in its support (that is, $\alpha=\sum_{\delta \in \operatorname{Supp} \alpha} \delta$ ), and nontypical otherwise.

It is easy to see that an active root $\alpha$ is typical if and only if it is contained in row 1 of Table 1 .

Lemma 33. Let $\alpha$ be a nontypical maximal active root. Then the simple root $\delta \in$ $F(\alpha) \cap \operatorname{Supp} \alpha$ marked in Table 2 by a star is a regular active root. (The notation in Table 2 is the same as in Table 1.)

TABLE 2

\begin{tabular}{|c|c|}
\hline Type of $\Delta(\alpha)$ & $\alpha$ \\
\hline $\mathrm{B}_{n}$ & $\alpha_{1}+\alpha_{2}+\cdots+\alpha_{n-1}+2 \alpha_{n}^{*}$ \\
\hline $\mathrm{C}_{n}$ & $2 \alpha_{1}^{*}+2 \alpha_{2}+\cdots+2 \alpha_{n-1}+\alpha_{n}$ \\
\hline $\mathrm{F}_{4}$ & $2 \alpha_{1}^{*}+2 \alpha_{2}+\alpha_{3}+\alpha_{4}$ \\
\hline $\mathrm{G}_{2}$ & $2 \alpha_{1}^{*}+\alpha_{2}$ \\
\hline $\mathrm{G}_{2}$ & $3 \alpha_{1}^{*}+\alpha_{2}$ \\
\hline
\end{tabular}

Proof. Assume that the active root $\delta$ is not regular. Then there exists an active root $\delta^{\prime} \neq \delta$ such that $\tau\left(\delta^{\prime}\right)=\tau(\delta)$. Since $\alpha-\delta \in \Delta_{+}$, by Proposition 1 we obtain $\beta=$ $\delta^{\prime}+(\alpha-\delta) \in$ M. Meanwhile $\operatorname{Supp}(\alpha-\delta)=\operatorname{Supp} \alpha$; therefore, $\operatorname{Supp} \alpha \subset \operatorname{Supp} \beta$, which is impossible because the active roots $\alpha$ and $\beta$ are maximal.

Proposition 17. There exists an element $w \in W$ such that $\bar{w} H \bar{w}^{-1} \subset B$ and all the maximal active roots of the subgroup $\bar{w} H \bar{w}^{-1}$ are typical.

Proof. Set $m=m(H)=\sum$ ht $\delta$, where $\delta$ runs over all nontypical maximal active roots of $H$. Let us prove the assertion by induction on $m$. For $m=0$ there is nothing to prove. Assume that the assertion is proved for all $m<k$, where $k>0$, and prove it for $m=k$. Let $\alpha$ be a nontypical maximal active root. Consider the indecomposable component $\Delta^{0}$ of $\Delta$ containing $\alpha$, and let $\Pi^{0}$ denote its set of simple roots, $\Pi^{0}=\Pi \cap \Delta^{0}$. We assume that $\Pi^{0}=\left\{\alpha_{1}, \ldots, \alpha_{n}\right\}$, where $n=\left|\Pi^{0}\right|$. We also set $\Delta_{+}^{0}=\Delta_{+} \cap \Delta^{0}$. Let $\delta \in \operatorname{Supp} \alpha$ be the simple root marked in Table 2 by a star. By Lemma 33 the root $\delta$ is a regular active root. Consider the subgroup $H^{\prime}=\bar{r}_{\delta} H \bar{r}_{\delta}^{-1}$ and show that $m\left(H^{\prime}\right)<m(H)$. We point out that by Lemma 30(c) all nontypical maximal active roots of $H^{\prime}$ are contained in the set $r_{\delta}(\mathrm{M}(H) \backslash\{\delta\})$. Since $r_{\delta}(\beta)=\beta$ for all roots $\beta \in \mathrm{M}(H) \backslash\{\alpha\}$ orthogonal to $\delta$, it suffices to show that the remaining maximal active roots of $H$ (including $\alpha$ ) 
make a positive contribution to the difference $m(H)-m\left(H^{\prime}\right)$. We next consider all the possibilities for $\Delta^{0}$ and $\alpha$ case-by-case.

$1^{\circ} . \Delta^{0}$ has type $B_{n}$. It follows from Theorem 3 that the support of every nontypical active root contained in $\Delta_{+}^{0}$ contains the simple root $\alpha_{n}$. Hence $\alpha$ is the unique nontypical maximal active root contained in $\Delta_{+}^{0}$. Let $\alpha=\alpha_{l}+\alpha_{l+1}+\cdots+\alpha_{n-1}+2 \alpha_{n}$, where $1 \leq l \leq n-1$. Then $\delta=\alpha_{n}$. Next, $r_{\delta}(\alpha)=\alpha_{l}+\alpha_{l+1}+\cdots+\alpha_{n-1} \in \mathrm{M}\left(H^{\prime}\right)$ is a typical root; therefore the contribution of the root $\alpha$ to the difference $m(H)-m\left(H^{\prime}\right)$ is equal to ht $\alpha$. If a root $\beta \in \mathrm{M}(H) \backslash\{\alpha\}$ is not orthogonal to $\delta$, then by Propositions 4 and 5 we obtain that $l=n-1, \beta=\alpha_{p}+\alpha_{p+1}+\cdots+\alpha_{n-1}$, where $1 \leq p \leq n-2$, and $\pi(\beta)=\pi(\alpha)=\alpha_{n-1}$. In other words, possibility (E1) is realized for the roots $\alpha$ and $\gamma$, whence $2 \alpha_{n}=\alpha-\alpha_{n-1} \in F(\alpha) \subset \Delta_{+}$, which is not the case. Therefore, $m(H)-m\left(H^{\prime}\right)=$ ht $\alpha>0$.

$2^{\circ} . \Delta^{0}$ has type $C_{n}$. It follows from Theorem 3 that the support of every nontypical active root contained in $\Delta_{+}^{0}$ contains the simple root $\alpha_{n}$. Hence $\alpha$ is the unique nontypical maximal active root contained in $\Delta_{+}^{0}$. Let $\alpha=2 \alpha_{l}+2 \alpha_{l+1}+\cdots+2 \alpha_{n-1}+\alpha_{n}$, where $1 \leq l \leq n-1$. Then $\delta=\alpha_{l}$. We have $r_{\delta}(\alpha)=2 \alpha_{l+1}+\cdots+2 \alpha_{n-1}+\alpha_{n}$, whence ht $\alpha-$ $\operatorname{ht}\left(r_{\delta}(\alpha)\right)=2$. If a root $\beta \in \mathrm{M}(H) \backslash\{\alpha\}$ is not orthogonal to $\delta$, then by Propositions 4 and 5 we obtain that $\operatorname{Supp} \beta \subset\left\{\alpha_{1}, \ldots, \alpha_{l}\right\}$. Hence each of the roots $\beta$ and $r_{\delta}(\beta)$ is typical. Thus, $m(H)-m\left(H^{\prime}\right)=2>0$.

$3^{\circ} . \Delta^{0}$ has type $\mathrm{F}_{4}$. By Theorem 3 there are four possible cases; we consider each of them separately.

Case 1. $\alpha=2 \alpha_{2}+\alpha_{3}$. Then $\delta=\alpha_{2}$. We have $r_{\delta}(\alpha)=\alpha_{3}$, so the contribution of the root $\alpha$ to the difference $m(H)-m\left(H^{\prime}\right)$ is equal to ht $\alpha=3$. If a root $\beta \in \mathrm{M}(H) \backslash\{\alpha\}$ is not orthogonal to $\delta$, then by Propositions 4 and 5 we obtain that either $\operatorname{Supp} \beta \subset\left\{\alpha_{1}, \alpha_{2}\right\}$ or $\beta=\alpha_{3}+\alpha_{4}$. In the first case, each of the roots $\beta$ and $r_{\delta}(\beta)$ is typical. In the second case, the possibility (E1) is realized for the roots $\alpha$ and $\beta$, and so $2 \alpha_{2}=\alpha-\alpha_{3} \in \Psi \subset \Delta_{+}$, which is not the case. Therefore, $m(H)-m\left(H^{\prime}\right)=3>0$.

Case 2. $\alpha=2 \alpha_{2}+\alpha_{3}+\alpha_{4}$. Then $\delta=\alpha_{2}$. Since $r_{\delta}(\alpha)=\alpha_{3}+\alpha_{4}$ is a typical root, the contribution of the root $\alpha$ to the difference $m(H)-m\left(H^{\prime}\right)$ is equal to ht $\alpha=4$. If a root $\beta \in \mathrm{M}(H) \backslash\{\alpha\}$ is not orthogonal to $\delta$, then by Propositions 4 and 5 we obtain that $\operatorname{Supp} \beta \subset\left\{\alpha_{1}, \alpha_{2}\right\}$, whence each of the $\operatorname{roots} \beta$ and $r_{\delta}(\beta)$ is typical. Thus, $m(H)-m\left(H^{\prime}\right)=4>0$.

Case 3. $\alpha=2 \alpha_{1}+2 \alpha_{2}+\alpha_{3}$. Then $\delta=\alpha_{1}$. We have $r_{\delta}(\alpha)=2 \alpha_{2}+\alpha_{3}$, so ht $\alpha-$ ht $\left(r_{\delta}(\alpha)\right)=2$. It follows from Propositions 4 and [5] that all roots in $\mathrm{M}(H) \backslash\{\alpha\}$ are orthogonal to $\delta$. Therefore, $m(H)-m\left(H^{\prime}\right)=2>0$.

Case 4. $\alpha=2 \alpha_{1}+2 \alpha_{2}+\alpha_{3}+\alpha_{4}$. Then $\delta=\alpha_{1}$. We have $r_{\delta}(\alpha)=2 \alpha_{2}+\alpha_{3}+\alpha_{4}$, and so ht $\alpha-\operatorname{ht}\left(r_{\delta}(\alpha)\right)=2$. Clearly, all roots in $\mathrm{M}(H) \backslash\{\alpha\}$ are orthogonal to the root $\delta$. Hence $m(H)-m\left(H^{\prime}\right)=2>0$.

$4^{\circ}$. $\Delta^{0}$ has type $\mathrm{G}_{2}$. By Theorem 3 we have $\alpha=2 \alpha_{1}+\alpha_{2}$ or $\alpha=3 \alpha_{1}+\alpha_{2}$. In both cases, $\delta=\alpha_{1}$. For $\alpha=2 \alpha_{1}+\alpha_{2}$ and $\alpha=3 \alpha_{1}+\alpha_{2}$ we have $r_{\delta}(\alpha)=\alpha_{1}+\alpha_{2}$ and $r_{\delta}(\alpha)=\alpha_{2}$, respectively; therefore the contribution of the root $\alpha$ to the difference $m(H)-m\left(H^{\prime}\right)$ is equal to ht $\alpha$. Clearly, all roots in $\mathrm{M}(H) \backslash\{\alpha\}$ are orthogonal to $\delta$. Therefore, $m(H)-m\left(H^{\prime}\right)=$ ht $\alpha \geq 3>0$.

Thus, we see that $m(H)>m\left(H^{\prime}\right)$. By the induction hypothesis there exists an element $w^{\prime} \in w$ such that $H^{\prime \prime}=\bar{w}^{\prime} H^{\prime} \bar{w}^{\prime-1} \subset B$ and all maximal active roots of the subgroup $H^{\prime \prime}$ are typical. Then it is easy to see that $\bar{w} H \bar{w}^{-1}=t H^{\prime \prime} t^{-1}$ for $w=w^{\prime} r_{\delta}$ and some $t \in T$, which completes the proof.

Remark 7. If all maximal active roots of $H$ are typical, then all active roots of $H$ are typical. 
Remark 8. If all roots in $\mathrm{M}(H)$ are typical, then for two distinct roots in $\mathrm{M}(H)$ condition (E1) is equivalent to the condition

(E1') Supp $\alpha \cap \operatorname{Supp} \beta=\{\gamma\}$, where $\gamma=\pi(\alpha)=\pi(\beta)$, and the root $\gamma$ is terminal with respect to $\operatorname{Supp} \alpha$ and $\operatorname{Supp} \beta$.

The following proposition is the next step in simplifying the set of combinatorial data of a connected solvable spherical subgroup standardly embedded in $B$.

Proposition 18. Suppose that all maximal active roots of $H$ are typical. Then there exists an element $w \in W$ such that $H^{\prime}=\bar{w} H \bar{w}^{-1} \subset B$, all maximal active roots of $H^{\prime}$ are typical, and for any two maximal active roots $\alpha, \beta$ of $H$ one of the following three possibilities is realized: (D0), (E1'), or

$\left(\mathrm{E}^{\prime}\right)$ the diagram $\Sigma(\operatorname{Supp} \alpha \cup \operatorname{Supp} \beta)$ has the form as in Figure 1 (where $p, q, r \geq 1$ are some numbers $), \alpha=\alpha_{1}+\cdots+\alpha_{p}+\gamma_{0}+\gamma_{1}+\cdots+\gamma_{r}, \beta=\beta_{1}+\cdots+\beta_{q}+\gamma_{0}+\gamma_{1}+\cdots+\gamma_{r}$, and $\pi(\alpha)=\pi(\beta)=\gamma_{r}$.

Before we prove this proposition, we need several auxiliary lemmas.

Lemma 34. If a simple root $\delta$ is active and is contained in the supports of at least two distinct maximal active roots, then $\delta$ is a regular active root.

Proof. Let $\alpha, \beta$ be distinct maximal active roots such that $\delta \in \operatorname{Supp} \alpha \cap \operatorname{Supp} \beta$. Assume that there exists an active root $\delta^{\prime} \neq \delta$ such that $\tau\left(\delta^{\prime}\right)=\tau(\delta)$. Then by Proposition 1 we obtain $\alpha^{\prime}=\delta^{\prime}+(\alpha-\delta) \in \mathrm{M}, \beta^{\prime}=\delta^{\prime}+(\beta-\delta) \in \mathrm{M}$, and $\alpha^{\prime} \neq \beta^{\prime}$. Since $\delta^{\prime} \neq \delta$, it follows that $\alpha^{\prime} \neq \alpha$ and $\beta^{\prime} \neq \beta$. But then the linear dependence $\alpha+\beta^{\prime}=\beta+\alpha^{\prime}$ contradicts Lemma 3 even if some of the roots $\alpha, \beta, \alpha^{\prime}, \beta^{\prime}$ coincide with each other.

Lemma 35. Suppose that $\alpha$ is a typical maximal active root such that $|\operatorname{Supp} \alpha| \geq 2$ and the root system $\Delta(\alpha)$ has type different from $\mathrm{G}_{2}$. Let $\delta \in \operatorname{Supp} \alpha$ be a regular active root.

(a) If in the diagram $\operatorname{Supp} \alpha$ the node $\delta$ is incident to a double edge with the arrow directed to $\delta$, then $r_{\delta}(\alpha)=\alpha$.

(b) Otherwise, $r_{\delta}(\alpha)=\alpha-\delta$.

Proof. By Corollary 7 the root $\delta$ is terminal with respect to $\operatorname{Supp} \alpha$. The rest of the proof is obtained by direct verification.

Lemma 36. Suppose that $\alpha, \beta$ are distinct maximal active roots and an active simple root $\delta$ is such that $\delta \in \operatorname{Supp} \alpha \cap \operatorname{Supp} \beta$. Let $\alpha_{0}$ denote the (unique) node of the diagram $\Sigma(\operatorname{Supp} \alpha)$ that is connected by an edge to the node $\delta$. Then for every maximal active root $\gamma \neq \alpha$ with $\alpha_{0} \in \operatorname{Supp} \gamma$ we have $\delta \in \operatorname{Supp} \gamma$.

Proof. We argue by contradiction. Assume that a maximal active root $\gamma \neq \alpha$ is such that $\alpha_{0} \in \operatorname{Supp} \gamma$ but $\delta \notin \operatorname{Supp} \gamma$. By Corollary 7 the root $\delta$ is terminal with respect to $\operatorname{Supp} \alpha$. If $\alpha_{0}$ is also terminal with respect to $\operatorname{Supp} \alpha$, then $|\operatorname{Supp} \alpha|=2$ in contradiction to Proposition 6. Now assume that $\alpha_{0}$ is not terminal with respect to $\operatorname{Supp} \alpha$. Then the set $\operatorname{Supp} \alpha \cap \operatorname{Supp} \gamma$ contains at least two elements. Since $\delta \notin \operatorname{Supp} \gamma$, in view of Propositions 4 and 5, possibility (E2) is realized for the roots $\alpha$ and $\gamma$, and $\operatorname{Supp} \alpha \cap \operatorname{Supp} \gamma=\operatorname{Supp} \alpha \backslash\{\delta\}$. Then $\operatorname{Supp} \alpha \subset \operatorname{Supp} \beta \cup \operatorname{Supp} \gamma$, and we again obtain a contradiction to Proposition 6 .

For any two distinct maximal active roots $\alpha, \beta$ of the subgroup $H$ we introduce a quantity $f(\alpha, \beta)=f(H, \alpha, \beta)$ as follows. If the set $\operatorname{Supp} \alpha \cap \operatorname{Supp} \beta$ contains an active simple root, then we set $f(\alpha, \beta)=|\operatorname{Supp} \alpha \cap \operatorname{Supp} \beta|$. Otherwise we set $f(\alpha, \beta)=0$.

Lemma 37. Suppose that a regular active simple root $\delta$ and a maximal active root $\alpha$ are such that in the diagram $\Sigma(\Pi)$ the node $\delta$ is connected by an edge to none of 
the nodes of the set $\operatorname{Supp} \alpha$. Then for every maximal active root $\beta \notin\{\alpha, \delta\}$ we have $f(H, \alpha, \beta)=f\left(\bar{r}_{\delta} H \bar{r}_{\delta}^{-1}, r_{\delta}(\alpha), r_{\delta}(\beta)\right)$.

Proof. It follows from the hypothesis that the root $\delta$ is not contained in the set Supp $\alpha$ and is orthogonal to all roots contained in this set, so $r_{\delta}(\alpha)=\alpha$. Then for every maximal active root $\beta$ we have $\operatorname{Supp} \alpha \cap \operatorname{Supp} \beta=\operatorname{Supp} r_{\delta}(\alpha) \cap \operatorname{Supp} r_{\delta}(\beta)$. To complete the proof it remains to observe that for a root $\delta^{\prime} \in \operatorname{Supp} \alpha \cap \operatorname{Supp} \beta$ the conditions $\delta^{\prime} \in \Psi(H)$ and $\delta^{\prime} \in \Psi\left(\bar{r}_{\delta} H \bar{r}_{\delta}^{-1}\right)$ are equivalent.

Proof of Proposition 18, Set $f(H)=\sum f(H, \alpha, \beta)$, where the sum is taken over all (unordered) pairs of distinct maximal active roots of $H$. We prove the assertion by induction on $f(H)$.

If $f(H)=0$, then for any two distinct roots $\alpha, \beta \in \mathrm{M}(H)$ the set $\operatorname{Supp} \alpha \cap \operatorname{Supp} \beta$ contains no active roots. But by Propositions 4 and 5 this means precisely that one of the possibilities (D0), $\left(\mathrm{E} 1^{\prime}\right)$, or $\left(\mathrm{E} 2^{\prime}\right)$ is realized for the roots $\alpha, \beta$.

Assume that for some $k>0$ the assertion holds for all subgroups $H$ with $f(H)<k$; we now prove it for $f(H)=k$. Let $\delta$ be an active simple root contained in the supports of at least two distinct maximal active roots of $H$. Then the root $\delta$ is regular by Lemma 34, Consider the subgroup $H_{0}=\bar{r}_{\delta} H \bar{r}_{\delta}^{-1}$; we claim that $f\left(H_{0}\right)<f(H)$ and all roots in the set $\mathrm{M}\left(H_{0}\right)$ are typical. Let $\Delta^{0}$ denote the indecomposable component of the root system $\Delta$ containing $\delta$. Clearly, $\Delta^{0}$ has type different from $\mathrm{G}_{2}$. Using Lemma 30(c) it is easy to see that only pairs of roots in $\mathrm{M}\left(H_{0}\right)$ that are contained in the set $r_{\delta}(\mathrm{M}(H))$ can make a nonzero contribution to $f\left(H_{0}\right)$. Note that if, for a root $\alpha \in \mathrm{M}(H)$, in the diagram $\Sigma\left(\Delta^{0}\right)$ the node $\delta$ is connected by an edge to none of the nodes in the set $\operatorname{Supp} \alpha$, then $r_{\delta}(\alpha)=\alpha$, which implies that the root $r_{\delta}(\alpha)$ is typical. Next, if distinct roots $\alpha, \beta \in \mathrm{M}(H)$ are such that $\delta \in \operatorname{Supp} \alpha \cap \operatorname{Supp} \beta$, then by Propositions 4 and 5 , one of the possibilities (D1), $(\mathrm{D} 2)$, or $(\mathrm{E} 2)$ is realized for them (and if (E2) is realized, then (E2') is not). In view of Lemma 35 and the condition that in the diagram $\Sigma(\Pi)$ the node $\delta$ is incident to at most one double edge, in all the cases we obtain $f\left(H_{0}, r_{\delta}(\alpha), r_{\delta}(\beta)\right)=f(H, \alpha, \beta)-1$. Moreover, each of the roots $r_{\delta}(\alpha)$ and $r_{\delta}(\beta)$ is typical. By Lemma 36, to proceed further we have to look at two cases.

Case 1. For every root $\alpha \in \mathrm{M}(H)$ with $\delta \notin \operatorname{Supp} \alpha$, in the diagram $\Sigma(\Pi)$ the node $\delta$ is connected by an edge to none of the nodes in the set $\operatorname{Supp} \alpha$. Then, for any such root $\alpha$, by Lemma 37 we see that the equality $f(H, \alpha, \beta)=f\left(H_{0}, r_{\delta}(\alpha), r_{\delta}(\beta)\right)$ holds for every root $\beta \in \mathrm{M}(H) \backslash\{\alpha\}$.

Case 2. The diagram $\Sigma\left(\Pi \cap \Delta^{0}\right)$ has the form as in Figure 1 (for some $p, q, r \geq 1$ ), $\delta=\gamma_{r^{\prime}}$, where $0 \leq r^{\prime} \leq r-1$, and the set $\mathrm{M}(H)$ contains the roots $\alpha=\alpha_{1}+\cdots+\alpha_{p^{\prime}}+$ $\gamma_{0}+\gamma_{1}+\cdots+\gamma_{r^{\prime}}, \beta=\beta_{1}+\cdots+\beta_{q^{\prime}}+\gamma_{0}+\gamma_{1}+\cdots+\gamma_{r^{\prime}}, \gamma=\gamma_{r^{\prime}+1}+\gamma_{r^{\prime}+2}+\cdots+\gamma_{r^{\prime \prime}}$ for some $p^{\prime}, q^{\prime}, r^{\prime \prime}$, where $1 \leq p^{\prime} \leq p, 1 \leq q^{\prime} \leq q, r^{\prime}+1 \leq r^{\prime \prime} \leq r$. By Propositions 4, 5, and 6, none of the maximal active roots of $H$ different from $\gamma$ contains $\gamma_{r^{\prime}+1}$ in its support. This fact and Lemma 36 imply that for every root $\alpha^{\prime} \in \mathrm{M}(H)$ different from $\alpha, \beta$, $\gamma$, the root $\delta$ is orthogonal to all roots in Supp $\alpha^{\prime}$. Then by Lemma 37 we obtain that the equality $f\left(H, \alpha^{\prime}, \beta^{\prime}\right)=f\left(H_{0}, r_{\delta}\left(\alpha^{\prime}\right), r_{\delta}\left(\beta^{\prime}\right)\right)$ holds for every root $\beta^{\prime} \in \mathrm{M}(H) \backslash\left\{\alpha^{\prime}\right\}$. Next, it is easy to verify that $f(H, \alpha, \gamma)=f\left(H_{0}, r_{\delta}(\alpha), r_{\delta}(\gamma)\right)=0, f(H, \beta, \gamma)=f\left(H_{0}, r_{\delta}(\beta), r_{\delta}(\gamma)\right)=0$, and each of the roots $r_{\delta}(\alpha), r_{\delta}(\beta), r_{\delta}(\gamma)$ is typical.

In both cases, $f\left(H_{0}\right)<f(H)$ and all roots in the set $\mathrm{M}\left(H_{0}\right)$ are typical. Then by the induction hypothesis there exists an element $w^{\prime} \in W$ such that the subgroup $H^{\prime}=\bar{w}^{\prime} H_{0} \bar{w}^{\prime-1}$ is standardly embedded in $B$, all roots in the set $\mathrm{M}\left(H^{\prime}\right)$ are typical, and one of the possibilities (D0), $\left(\mathrm{E} 1^{\prime}\right)$, or $\left(\mathrm{E} 2^{\prime}\right)$ is realized for any two distinct roots $\alpha, \beta \in \mathrm{M}\left(H^{\prime}\right)$. Then the element $w=w^{\prime} r_{\delta}$ is the required one. 
Definition 10. The set $\Upsilon(H)=(S, \mathrm{M}, \pi, \sim)$, as well as its subset $\Upsilon_{0}(H)=(\mathrm{M}, \pi, \sim)$, is said to be reduced if the following conditions hold:

$\left(\mathrm{A}^{\prime}\right) \alpha=\sum_{\delta \in \operatorname{Supp} \alpha} \delta$ for every root $\alpha \in \mathrm{M}$, and $\pi(\alpha) \in \operatorname{Supp} \alpha$;

$\left(\mathrm{D}^{\prime}\right)$ if $\alpha, \beta \in \mathrm{M}$ and $\alpha \nsim \beta$, then $\operatorname{Supp} \alpha \cap \operatorname{Supp} \beta=\varnothing$;

$\left(\mathrm{E}^{\prime}\right)$ if $\alpha, \beta \in \mathrm{M}$ and $\alpha \sim \beta$, then one of the possibilities (D0), (E1'), or (E2') is realized for the roots $\alpha, \beta$.

Propositions 17 and 18 imply the following theorem.

Theorem 7. Every connected solvable spherical subgroup $H \subset G$ is conjugate to a subgroup $H^{\prime}$ standardly embedded in $B$ such that the set $\Upsilon\left(H^{\prime}\right)$ is reduced.

6.2. In this subsection we prove the following theorem.

Theorem 8. Let $H, H^{\prime} \subset G$ be two connected solvable spherical subgroups standardly embedded in $B$ such that the sets $\Upsilon(H)$ and $\Upsilon\left(H^{\prime}\right)$ are reduced. Suppose that $H$ and $H^{\prime}$ are conjugate in $G$. Then there exists a chain of elementary transformations taking $H$ to $H^{\prime}$ such that for every intermediate subgroup $\widetilde{H}$ of this chain the set $\Upsilon(\widetilde{H})$ is also reduced.

Before we prove this theorem, let us prove an auxiliary lemma.

Lemma 38. Suppose that $\alpha \in \Psi(H)$, $|\operatorname{Supp} \alpha| \geq 2$, and $\delta \in F(\alpha) \cap \Pi$. In the diagram $\Sigma(\operatorname{Supp} \alpha)$, consider the (unique) node $\delta^{\prime}$ connected by an edge to $\delta$. Then $\delta^{\prime} \notin \Psi(H)$.

Proof. Assume that $\delta^{\prime} \in \Psi\left(H^{\prime}\right)$. Then by Corollary 7 both roots $\delta, \delta^{\prime}$ are terminal with respect to $\operatorname{Supp} \alpha$, whence $\operatorname{Supp} \alpha=\left\{\delta, \delta^{\prime}\right\}$. In this situation, the roots $\delta, \delta^{\prime}$ cannot be simultaneously active for $H$, and we have a contradiction.

Proof of Theorem 8 . We assume without loss of generality that the root system $\Delta$ is indecomposable. If $\Delta$ has type $\mathrm{G}_{2}$, then the assertion is easily verified by a case-by-case analysis of all possible sets $(\mathrm{M}, \pi, \sim)$ (there are 9 in total) and all chains of elementary transformations. Therefore we assume in what follows that $\Delta$ has type different from $\mathrm{G}_{2}$.

Consider the shortest chain of elementary transformations taking $H$ to $H^{\prime}$; we claim that it is the required one. Let $\delta_{1}, \delta_{2}, \ldots, \delta_{k}$ be the consecutive centres of elementary transformations of this chain. Since the length of the chain is minimal, we have $\delta_{i} \neq \delta_{i-1}$ for $i=1, \ldots, k-1$. We set $H_{0}=H$ and for $i=1, \ldots, k$ let $H_{i}$ denote the $i$ th intermediate subgroup: $H_{i}=\sigma_{i} H_{i-1} \sigma_{i}^{-1}$, where $\sigma_{i} \in N_{G}(T)$ is a representative of the reflection $r_{\delta_{i}}$ and $H_{k}=H^{\prime}$. Let $\mathrm{M}_{i}$ denote the set of maximal active roots of $H_{i}, i=0, \ldots, k$. We complete the proof in two steps.

Step 1. We prove that the set $\mathrm{M}_{i}$ consists of typical roots for all $i=1, \ldots, k-1$. Assume the converse: for some $j \in\{1, \ldots, k-1\}$ the set $\mathrm{M}_{j}$ contains a nontypical root $\alpha$, whereas for all $i<j$ the set $\mathrm{M}_{i}$ consists of typical roots. We assume without loss of generality that the number $j$ is maximal among all the shortest chains of elementary transformations taking $H$ to $H^{\prime}$. It is easy to see that in the diagram $\Sigma(\Pi)$ the node $\delta_{j}$ is incident to a double edge with the arrow directed to $\delta_{j}$. We claim that the nodes $\delta_{j}$ and $\delta_{j+1}$ are connected by an edge. Indeed, otherwise the elementary transformations with centres $\delta_{j}$ and $\delta_{j+1}$ commute; interchanging them results in a new chain in which the set $\mathrm{M}_{i}$ can contain a nontypical root only if $i \geq j+1$, which contradicts the choice of $j$. We now look at all the possibilities for $\Delta$.

$1^{\circ} . \Delta$ has type $\mathrm{B}_{n}$. Then $\alpha=\alpha_{l}+\alpha_{l+1}+\cdots+2 \alpha_{n}$, where $1 \leq l \leq n-1$. We have $\delta_{j+1}=\alpha_{n-1}$, which is impossible, since the root $\alpha_{n-1}$ cannot be active.

$2^{\circ} . \Delta$ has type $\mathrm{C}_{n}, n \geq 3$. Then $\alpha=2 \alpha_{n-1}+\alpha_{n}$. The root $\alpha_{n}$ is not active; therefore, $\delta_{j+1}=\alpha_{n-2}$. We prove by induction that $\delta_{j+l}=\alpha_{n-l-1}$ for all $l=1, \ldots, n-2$. This holds for $l=1$. We make the step from $l-1$ to $l$ as follows. Clearly, the set $\mathrm{M}_{j+l-1}$ 
contains the root $2 \alpha_{n-l}+2 \alpha_{n-l+1}+\cdots+2 \alpha_{n-1}+\alpha_{n}$. Therefore none of the roots $2 \alpha_{n-l+1}, \ldots, \alpha_{n-1}, \alpha_{n}$ can be active (with respect to $H_{j+l-1}$ ). If $\delta_{j+l} \neq \alpha_{n-l-1}$, then by taking the condition $\delta_{j+l} \neq \delta_{j+l-l}$ into account we see that the root $\delta_{j+1}$ is orthogonal to each of the roots $\alpha_{n-l}, \alpha_{n-l+1}, \ldots, \alpha_{n-1}$. Therefore by successively transposing neighbouring terms in our chain of elementary transformations we can transfer the $(j+l)$ th elementary transformation to the $j$ th place. As a result we obtain a new chain for which the set $\mathrm{M}_{i}$ can only contain a nontypical root for $i \geq j+1$, which contradicts the maximality of $j$. Thus, we have $\delta_{j+n-2}=\alpha_{1}$ and $\mathrm{M}_{j+n-2}=\left\{2 \alpha_{1}+\cdots+2 \alpha_{n-1}+\alpha_{n}\right\}$, whence the root $\delta_{j+n-1}$ can be none of the simple roots, which is impossible.

$3^{\circ} . \Delta$ has type $\mathrm{F}_{4}$. Then $\alpha=2 \alpha_{2}+\alpha_{3}$ or $\alpha=2 \alpha_{2}+\alpha_{3}+\alpha_{4}$. In both cases, the root $\alpha_{3}$ is not active; therefore $\delta_{j+1}=\alpha_{1}$. But then $\delta_{j+2}=\alpha_{4}$, and by successively transposing neighbouring terms in our chain of elementary transformations we can transfer the $(j+2)$ th elementary transformation to the $j$ th place. The new chain has the property that the set $\mathrm{M}_{i}$ can only contain nonrtypical roots for $i \geq j+1$, which contradicts the maximality of $j$.

Thus, in all the three cases we have obtained a contradiction; therefore the set $\mathrm{M}_{i}$ consists of typical roots for all $i=1, \ldots, k-1$.

Step 2. We now prove that for all $i=1, \ldots, k-1$, one of the possibilities (D0), (E1'), or $\left(\mathrm{E} 2^{\prime}\right)$ is realized for any two distinct roots in the set $\mathrm{M}_{i}$. To do this it is enough to prove that for all $i=1, \ldots, k-1$ every active simple root of the subgroup $H_{i}$ is contained in the support of exactly one root in $\mathrm{M}_{i}$. Assume the converse: for some $j \in\{1, \ldots, k-1\}$ there exists a root $\delta \in \Psi\left(H_{j}\right) \cap \Pi$ contained in the support of two distinct roots $\alpha, \beta \in \mathrm{M}_{j}$, whereas for all $i<j$ there are no active simple roots with the analogous property. We assume without loss of generality that the number $j$ is maximal among all the shortest chains of elementary transformations taking $H$ to $H^{\prime}$.

We claim that $\delta_{j}=\delta$. Assume that this is not the case and that $\delta_{j}=\nu \neq \delta$. Then $r_{\nu}(\alpha), r_{\nu}(\beta) \in \mathrm{M}_{j-1}$ by Lemma 30(c). We have $\nu \neq \delta$ and $\nu \neq \alpha-\delta$; therefore both roots $r_{\nu}(\delta)$ and $r_{\nu}(\alpha-\delta)$ are positive, and so $r_{\nu}(\delta) \in F\left(r_{\nu}(\alpha)\right)$. Similarly, $r_{\nu}(\delta) \in F\left(r_{\nu}(\beta)\right)$. Then any root in the (nonempty) set $F\left(r_{\nu}(\delta)\right) \cap \Pi$ is contained in the support of the maximal active roots $r_{\nu}(\alpha), r_{\nu}(\beta)$ of $H_{j-1}$, which contradicts the choice of $j$.

Thus, $\delta_{j}=\delta$. By repeating the analogous argument in step 1 word-for-word, we verify that in the diagram $\Sigma(\Pi)$ the nodes $\delta_{j}$ and $\delta_{j+1}$ are connected by an edge. There are two possible cases.

Case 1. Every node of the diagram $\Sigma(\Pi)$ connected by an edge to $\delta$ is contained in the support of a root $\alpha \in \mathrm{M}_{j}$ such that $\delta \in F(\alpha)$. In this case we obtain a contradiction in view of Lemma 38 .

Case 2. The diagram $\Sigma(\Pi)$ has the form as in Figure 1 (for some $p, q, r \geq 1$ ), $\delta=\gamma_{r^{\prime}}$, where $0 \leq r^{\prime} \leq r-1$, and the set $\mathrm{M}_{j}$ contains the roots $\alpha=\alpha_{1}+\cdots+\alpha_{p^{\prime}}+\gamma_{0}+\gamma_{1}+\cdots+\gamma_{r^{\prime}}$ and $\beta=\beta_{1}+\cdots+\beta_{q^{\prime}}+\gamma_{0}+\gamma_{1}+\cdots+\gamma_{r^{\prime}}$, where $1 \leq p^{\prime} \leq p, 1 \leq q^{\prime} \leq q$. By Lemma 38 we obtain $\delta_{j+1}=\gamma_{r^{\prime}+1}$. Applying inductive arguments similar to the arguments in case $2^{\circ}$ of step 1 , we see that $\delta_{j+i}=\gamma_{r^{\prime}+i}$ for all $i=1, \ldots, r-r^{\prime}$. Next, the root $\delta_{j+r-r^{\prime}+1}$ cannot be contained in the set $\operatorname{Supp} \alpha \cup \operatorname{Supp} \beta$; therefore it is orthogonal to each of the roots $\gamma_{r^{\prime}}, \gamma_{r^{\prime}+1}, \ldots, \gamma_{r}$. Therefore by successively transposing neighbouring terms in our chain of elementary transformations we can transfer the $\left(j+r-r^{\prime}+1\right)$ th elementary transformation to the $j$ th place. As a result we obtain a new chain such that for all $i \leq j$ every active simple root of $H_{i}$ is contained in the support of exactly one root in $\mathrm{M}_{i}$. We obtain a contradiction to the maximality of $j$.

Thus, in both cases we have arrived at a contradiction, which completes the proof of the theorem. 
6.3. Let $H \subset G$ be a connected solvable spherical subgroup standardly embedded in $B$ such that the set $\Upsilon(H)$ is reduced. Consider an elementary transformation $H \mapsto H^{\prime}=$ $\sigma_{\delta} H \sigma_{\delta}^{-1}$, where $\delta \in \Psi(H) \cap \Pi$ is a regular active simple root and $\sigma_{\delta} \in N_{G}(T)$ is a representative of the reflection $r_{\delta}$. In this subsection we shall find conditions on the root $\delta$ under which the set $\Upsilon_{0}\left(H^{\prime}\right)$ is reduced and different from the set $\Upsilon_{0}(H)$.

Note that the set $\Upsilon\left(H^{\prime}\right)$ is reduced if and only if the following two conditions hold:

(1) every root in $\mathrm{M}\left(H^{\prime}\right)$ is typical;

(2) for any two distinct roots $\alpha, \beta \in \mathrm{M}\left(H^{\prime}\right)$ the set $\operatorname{Supp} \alpha \cap \operatorname{Supp} \beta$ contains no active simple roots of $H^{\prime}$.

To proceed further we need the following notation:

$\mathrm{M}_{0}(H, \delta)$ is the set of roots $\alpha \in \mathrm{M}(H) \backslash\{\delta\}$ such that in the diagram $\Sigma(\Pi)$ the node $\delta$ is connected by an edge to none of the nodes in Supp $\alpha$;

$\mathrm{M}_{1}(H, \delta)$ is the set of roots $\alpha \in \mathrm{M}(H) \backslash\{\delta\}$ such that $\delta \notin \operatorname{Supp} \alpha$ and there exists a (unique) root $\gamma \in \operatorname{Supp} \alpha$ such that in the diagram $\Sigma(\Pi)$ the nodes $\delta$ and $\gamma$ are connected by an edge;

$\mathrm{M}_{11}(H, \delta)$ is the set of roots $\alpha \in \mathrm{M}_{1}(H, \delta)$ such that in the diagram $\Sigma(\Pi)$ the nodes $\delta$ and $\gamma$ are connected by a triple edge with the arrow directed to $\delta$;

$\mathrm{M}_{12}(H, \delta)$ is the set of roots $\alpha \in \mathrm{M}_{1}(H, \delta)$ such that in the diagram $\Sigma(\Pi)$ the nodes $\delta$ and $\gamma$ are connected by a double edge with the arrow directed to $\delta$;

$\mathrm{M}_{13}(H, \delta)=\mathrm{M}_{1}(H, \delta) \backslash\left(\mathrm{M}_{11}(H, \delta) \cup \mathrm{M}_{12}(H, \delta)\right)$;

$\mathrm{M}_{2}(H, \delta)$ is the set of roots $\alpha \in \mathrm{M}(H) \backslash\{\delta\}$ such that $\delta \in \operatorname{Supp} \alpha$;

$\mathrm{M}_{21}(H, \delta)$ is the set of roots $\alpha \in \mathrm{M}_{2}(H, \delta)$ such that in the diagram $\Sigma(\operatorname{Supp} \alpha)$ the node $\delta$ is incident to a triple edge with the arrow directed to $\delta$;

$\mathrm{M}_{22}(H, \delta)$ is the set of roots $\alpha \in \mathrm{M}_{2}(H, \delta)$ such that in the diagram $\Sigma(\operatorname{Supp} \alpha)$ the node $\delta$ is incident to a double edge with the arrow directed to $\delta$;

$\mathrm{M}_{23}(H, \delta)=\mathrm{M}_{2}(H, \delta) \backslash\left(\mathrm{M}_{21}(H, \delta) \cup \mathrm{M}_{22}(H, \delta)\right)$.

Note that we have disjoint unions

$$
\begin{aligned}
\mathrm{M}(H) & =\mathrm{M}_{0}(H, \delta) \cup \mathrm{M}_{1}(H, \delta) \cup \mathrm{M}_{2}(H, \delta), \\
\mathrm{M}_{1}(H, \delta) & =\mathrm{M}_{11}(H, \delta) \cup \mathrm{M}_{12}(H, \delta) \cup \mathrm{M}_{13}(H, \delta), \\
\mathrm{M}_{2}(H, \delta) & =\mathrm{M}_{21}(H, \delta) \cup \mathrm{M}_{22}(H, \delta) \cup \mathrm{M}_{23}(H, \delta) .
\end{aligned}
$$

Proposition 19. (a) The set $\Upsilon_{0}\left(H^{\prime}\right)$ coincides with the set $\Upsilon_{0}(H)$ if and only if

$$
\left|\mathrm{M}_{1}(H, \delta)\right|+\left|\mathrm{M}_{21}(H, \delta)\right|+\left|\mathrm{M}_{23}(H, \delta)\right|=0 .
$$

(b) The set $\Upsilon_{0}\left(H^{\prime}\right)$ contains a nontypical root if and only if

$$
\left|\mathrm{M}_{11}(H, \delta)\right|+\left|\mathrm{M}_{12}(H, \delta)\right|+\left|\mathrm{M}_{21}(H, \delta)\right| \geq 1 .
$$

(c) There exists a simple root in the set $\Psi\left(H^{\prime}\right)$ contained in the supports of at least two roots in $\mathrm{M}\left(H^{\prime}\right)$ if and only if $\left|\mathrm{M}_{1}(H, \delta)\right|+\left|\mathrm{M}_{22}(H, \delta)\right| \geq 2$.

Proof. (a) The condition $\left|\mathrm{M}_{1}(H, \delta)\right|+\left|\mathrm{M}_{21}(H, \delta)\right|+\left|\mathrm{M}_{23}(H, \delta)\right|=0$ is equivalent to the condition that $\delta$ is orthogonal to all roots in the set $\mathrm{M}(H) \backslash\{\delta\}$. If it holds, then, obviously, $\Upsilon_{0}\left(H^{\prime}\right)=\Upsilon_{0}(H)$. Conversely, suppose that $\Upsilon_{0}\left(H^{\prime}\right)=\Upsilon_{0}(H)$ but there exists a root $\alpha \in \mathrm{M}(H) \backslash\{\delta\}$ that is not orthogonal to $\delta$. Then we have $\alpha, r_{\delta}(\alpha) \in \mathrm{M}(H)$, whence the support of one of these roots is contained in the support of the other, which is impossible by Corollary 3 (c).

(b) The required assertion is obtained by a direct check.

(c) We use Lemma 37 to reduce the proof to a consideration of roots in the sets $\mathrm{M}_{1}(H, \delta)$ and $\mathrm{M}_{2}(H, \delta)$. Then the proof is completed by a direct check. 
Corollary 18. The set $\Upsilon_{0}\left(H^{\prime}\right)$ is reduced and distinct from the set $\Upsilon_{0}(H)$ if and only if the following conditions hold:

(1) $\left|\mathrm{M}_{13}(H, \delta)\right|+\left|\mathrm{M}_{23}(H, \delta)\right| \geq 1$;

(2) $\left|\mathrm{M}_{11}(H, \delta)\right|+\left|\mathrm{M}_{12}(H, \delta)\right|+\left|\mathrm{M}_{21}(H, \delta)\right|=0$;

(3) $\left|\mathrm{M}_{13}(H, \delta)\right|+\left|\mathrm{M}_{22}(H, \delta)\right| \leq 1$.

\section{$\S 7$. EXAMPLES}

In this section, to illustrate the theory presented in this paper, for all simple groups $G$ of rank at most 4 we list, up to conjugacy, all saturated solvable spherical subgroups of $G$, that is, solvable spherical subgroups $H \subset G$ such that $H=N_{G}(H)^{0}$ (see $\S 5.5$ ). Thereby we also list, up to conjugacy, all unipotent subgroups of $G$ that are the unipotent radicals of connected solvable spherical subgroups of $G$ (see Corollary 17 and Proposition 16).

For a fixed group $G$ the enumeration is carried out in two steps.

Step 1 . We enumerate all reduced sets $(\mathrm{M}, \pi, \sim)$ (where $\mathrm{M} \subset \Delta_{+}$is a subset, $\pi: \mathrm{M} \rightarrow \Pi$ is a map and $\sim$ is an equivalence relation on $\mathrm{M})$. We recall that reduced sets are characterized by satisfying conditions $\left(\mathrm{A}^{\prime}\right),\left(\mathrm{D}^{\prime}\right),\left(\mathrm{E}^{\prime}\right)$ and $(\mathrm{C})$ (see Proposition 16 and Theorem [7).

Step 2. For every set $(\mathrm{M}, \pi, \sim)$ in the list obtained at step 1 , we indicate all other sets in this list that can be obtained from the set $(\mathrm{M}, \pi, \sim)$ by chains of elementary transformations (see Theorem 8, as well as Propositions 15, 14 and Corollary 18).

Note that the procedure described in step 1 depends only on the underlying graph of the diagram $\Sigma(\Pi)$, that is, the graph obtained from $\Sigma(\Pi)$ by replacing each multiple edge by a nonoriented simple edge. In this connection, it is convenient to perform the procedure in step 1 simultaneously for all groups $G$ whose underlying graphs of the corresponding diagrams $\Sigma(\Pi)$ are the same.

The procedure for enumerating all reduced sets for a given group $G$ can be shortened as follows. Let $(\mathrm{M}, \pi, \sim)$ be a reduced set. We set $\operatorname{Supp} \mathrm{M}=\bigcup_{\delta \in \mathrm{M}} \operatorname{Supp} \delta$. Note that the set Supp M is invariant under an elementary transformation. Furthermore, the procedure of enumerating all reduced sets $(\mathrm{M}, \pi, \sim)$ having a given set Supp M depends only on the diagram $\Sigma(\operatorname{Supp} M)$. In this connection, for a given group $G$, the original problem reduces to the following: for every subset $\Pi^{\prime} \subset \Pi$ perform steps 1,2 , and at step 1 enumerate only those reduced sets $(\mathrm{M}, \pi, \sim)$ for which $\operatorname{Supp} M=\Pi^{\prime}$. The advantage of this approach consists in that for any two subsets $\Pi^{\prime}, \Pi^{\prime \prime} \subset \Pi$ with $\Sigma\left(\Pi^{\prime}\right) \simeq \Sigma\left(\Pi^{\prime \prime}\right)$ the procedure of enumerating all reduced sets $(M, \pi, \sim)$ with $\operatorname{Supp} M=\Pi^{\prime}$ and $\operatorname{Supp} M=\Pi^{\prime \prime}$ can be performed simultaneously.

Let $(\mathrm{M}, \pi, \sim)$ be a reduced set. We consider the corresponding saturated solvable spherical subgroup $H \subset G$ standardly embedded in $B$. Let $c(S)$ denote the codimension in $T$ of the maximal torus $S=H \cap T$ of $H$, and let $c(N)$ denote the codimension in $U$ of the unipotent radical $N=H \cap U$ of $H$. We have $c(S)=|\mathrm{M}|-\mu$, where $\mu$ is the number of equivalence classes in the set $\mathrm{M}$, and $c(N)$ is equal to the number of equivalence classes in the set $\Psi$; see $\oint 4.2$. Note that $c(S)+c(N)=|\operatorname{Supp} \mathrm{M}|$, whence in the case $\operatorname{Supp} \mathrm{M}=\Pi$ we obtain $c(S)+c(N)=\operatorname{rk} G$. In particular, in the latter case we have $\operatorname{dim} H=\operatorname{dim} U$ and $H$ is a spherical subgroup of $G$ of minimal dimension.

Let $d_{0}(G)$ denote the number of conjugacy classes of saturated solvable spherical subgroups of $G$ that correspond to reduced sets (M, $\pi, \sim)$ with Supp M $=\Pi$.

Now, for all groups $G$ such that the diagrams $\Sigma(\Pi)$ are subdiagrams of connected Dynkin diagrams of rank at most 4 , we enumerate all reduced sets $(\mathrm{M}, \pi, \sim)$ with Supp $\mathrm{M}=\Pi$. Formally, we include the case $\operatorname{rk} G=0$ in our consideration; it corresponds to the situation where $\mathrm{M}=\varnothing$. In each case we also give the values of $c(S)$ and $c(N)$. Besides, we indicate the values of $d_{0}(G)$ for all groups $G$ under consideration. 
rk $G=0$. We have $\mathrm{M}=\varnothing, c(S)=c(N)=0, d_{0}(\varnothing)=1$.

$\operatorname{rk} G=1$. We have $\mathrm{M}=\Delta_{+}, c(S)=0, c(N)=1, d_{0}\left(\mathrm{~A}_{1}\right)=1$.

$\operatorname{rk} G=2$, the underlying graph is $\mathrm{A}_{1} \times \mathrm{A}_{1}$. We have $\mathrm{M}=\Pi=\left\{\alpha_{1}, \alpha_{2}\right\}$ and either $\alpha_{1} \not \alpha_{2}($ then $c(S)=0, c(N)=2)$ or $\alpha_{1} \sim \alpha_{2}($ then $c(S)=c(N)=1) ; d_{0}\left(\mathrm{~A}_{1} \times \mathrm{A}_{1}\right)=2$.

$\operatorname{rk} G=2$, the underlying graph is $\mathrm{A}_{2}$. The results are presented in Table 4 .

rk $G=3$, the underlying graph is $\mathrm{A}_{1} \times \mathrm{A}_{1} \times \mathrm{A}_{1}$. The results are presented in Table 5 . $\operatorname{rk} G=3$, the underlying graph is $\mathrm{A}_{1} \times \mathrm{A}_{2}$. The results are presented in Table 6 ,

$\operatorname{rk} G=3$, the underlying graph is $\mathrm{A}_{3}$. The results are presented in Table 7 .

$\operatorname{rk} G=4$, the underlying graph is $\mathrm{A}_{4}$. The results are presented in Table 8

$\operatorname{rk} G=4$, the underlying graph is $\mathrm{D}_{4}$. The results are presented in Table 9 .

Having known the values $d_{0}(G)$ for all the groups $G$ considered above, for every simple group $G$ of rank at most 4 we can determine the value $d(G)$, which is equal to the number of conjugacy classes in $G$ of saturated solvable spherical subgroups and also to the number of conjugacy classes in $G$ of unipotent radicals of connected solvable spherical subgroups. Below we present the corresponding calculations and Table 3 summarizing them.

$$
\begin{aligned}
& d\left(\mathrm{~A}_{1}\right)=d_{0}(\varnothing)+d_{0}\left(\mathrm{~A}_{1}\right)=1+1=2 . \\
& d\left(\mathrm{~A}_{2}\right)=d_{0}(\varnothing)+2 d_{0}\left(\mathrm{~A}_{1}\right)+d_{0}\left(\mathrm{~A}_{2}\right)=1+2 \cdot 1+2=5 . \\
& d\left(\mathrm{~B}_{2}\right)=d_{0}(\varnothing)+2 d_{0}\left(\mathrm{~A}_{1}\right)+d_{0}\left(\mathrm{~B}_{2}\right)=1+2 \cdot 1+3=6 . \\
& d\left(\mathrm{G}_{2}\right)=d_{0}(\varnothing)+2 d_{0}\left(\mathrm{~A}_{1}\right)+d_{0}\left(\mathrm{G}_{2}\right)=1+2 \cdot 1+3=6 . \\
& d\left(\mathrm{~A}_{3}\right)=d_{0}(\varnothing)+3 d_{0}\left(\mathrm{~A}_{1}\right)+d_{0}\left(\mathrm{~A}_{1} \times \mathrm{A}_{1}\right)+2 d_{0}\left(\mathrm{~A}_{2}\right)+d_{0}\left(\mathrm{~A}_{3}\right)=1+3 \cdot 1+2+2 \cdot 2+8=18 . \\
& d\left(\mathrm{~B}_{3}\right)=d_{0}(\varnothing)+3 d_{0}\left(\mathrm{~A}_{1}\right)+d_{0}\left(\mathrm{~A}_{1} \times \mathrm{A}_{1}\right)+d_{0}\left(\mathrm{~A}_{2}\right)+d_{0}\left(\mathrm{~B}_{2}\right)+d_{0}\left(\mathrm{~B}_{3}\right)=1+3 \cdot 1+2+2+3+11=22 . \\
& d\left(\mathrm{C}_{3}\right)=d_{0}(\varnothing)+3 d_{0}\left(\mathrm{~A}_{1}\right)+d_{0}\left(\mathrm{~A}_{1} \times \mathrm{A}_{1}\right)+d_{0}\left(\mathrm{~A}_{2}\right)+d_{0}\left(\mathrm{~B}_{2}\right)+d_{0}\left(\mathrm{C}_{3}\right)=1+3 \cdot 1+2+2+3+10=21 . \\
& d\left(\mathrm{~A}_{4}\right)=d_{0}(\varnothing)+4 d_{0}\left(\mathrm{~A}_{1}\right)+3 d_{0}\left(\mathrm{~A}_{1} \times \mathrm{A}_{1}\right)+3 d_{0}\left(\mathrm{~A}_{2}\right)+2 d_{0}\left(\mathrm{~A}_{1} \times \mathrm{A}_{2}\right)+2 d_{0}\left(\mathrm{~A}_{3}\right)+d_{0}\left(\mathrm{~A}_{4}\right)= \\
& 1+4 \cdot 1+3 \cdot 2+3 \cdot 2+2 \cdot 5+2 \cdot 8+31=74 . \\
& d\left(\mathrm{~B}_{4}\right)=d_{0}(\varnothing)+4 d_{0}\left(\mathrm{~A}_{1}\right)+3 d_{0}\left(\mathrm{~A}_{1} \times \mathrm{A}_{1}\right)+2 d_{0}\left(\mathrm{~A}_{2}\right)+d_{0}\left(\mathrm{~B}_{2}\right)+d_{0}\left(\mathrm{~A}_{1} \times \mathrm{A}_{2}\right)+d_{0}\left(\mathrm{~A}_{1} \times\right. \\
& \left.\mathrm{B}_{2}\right)+d_{0}\left(\mathrm{~A}_{3}\right)+d_{0}\left(\mathrm{~B}_{3}\right)+d_{0}\left(\mathrm{~B}_{4}\right)=1+4 \cdot 1+3 \cdot 2+2 \cdot 2+3+5+7+8+11+42=91 . \\
& d\left(\mathrm{C}_{4}\right)=d_{0}(\varnothing)+4 d_{0}\left(\mathrm{~A}_{1}\right)+3 d_{0}\left(\mathrm{~A}_{1} \times \mathrm{A}_{1}\right)+2 d_{0}\left(\mathrm{~A}_{2}\right)+d_{0}\left(\mathrm{~B}_{2}\right)+d_{0}\left(\mathrm{~A}_{1} \times \mathrm{A}_{2}\right)+d_{0}\left(\mathrm{~A}_{1} \times\right. \\
& \left.\mathrm{B}_{2}\right)+d_{0}\left(\mathrm{~A}_{3}\right)+d_{0}\left(\mathrm{C}_{3}\right)+d_{0}\left(\mathrm{C}_{4}\right)=1+4 \cdot 1+3 \cdot 2+2 \cdot 2+3+5+7+8+10+38=86 . \\
& d\left(\mathrm{D}_{4}\right)=d_{0}(\varnothing)+4 d_{0}\left(\mathrm{~A}_{1}\right)+3 d_{0}\left(\mathrm{~A}_{1} \times \mathrm{A}_{1}\right)+3 d_{0}\left(\mathrm{~A}_{2}\right)+d_{0}\left(\mathrm{~A}_{1} \times \mathrm{A}_{1} \times \mathrm{A}_{1}\right)+3 d_{0}\left(\mathrm{~A}_{3}\right)+d_{0}\left(\mathrm{D}_{4}\right)= \\
& 1+4 \cdot 1+3 \cdot 2+3 \cdot 2+5+3 \cdot 8+40=86 . \\
& d\left(\mathrm{~F}_{4}\right)=d_{0}(\varnothing)+4 d_{0}\left(\mathrm{~A}_{1}\right)+3 d_{0}\left(\mathrm{~A}_{1} \times \mathrm{A}_{1}\right)+2 d_{0}\left(\mathrm{~A}_{2}\right)+d_{0}\left(\mathrm{~B}_{2}\right)+2 d_{0}\left(\mathrm{~A}_{1} \times \mathrm{A}_{2}\right)+d_{0}\left(\mathrm{~B}_{3}\right)+ \\
& d_{0}\left(\mathrm{C}_{3}\right)+d_{0}\left(\mathrm{~F}_{4}\right)=1+4 \cdot 1+3 \cdot 2+2 \cdot 2+3+2 \cdot 5+11+10+38=87 .
\end{aligned}
$$

TABLE 3

\begin{tabular}{|c|c|c|c|c|c|c|c|c|c|c|c|c|}
\hline Type of $G$ & $\mathrm{~A}_{1}$ & $\mathrm{~A}_{2}$ & $\mathrm{~B}_{2}$ & $\mathrm{G}_{2}$ & $\mathrm{~A}_{3}$ & $\mathrm{~B}_{3}$ & $\mathrm{C}_{3}$ & $\mathrm{~A}_{4}$ & $\mathrm{~B}_{4}$ & $\mathrm{C}_{4}$ & $\mathrm{D}_{4}$ & $\mathrm{~F}_{4}$ \\
\hline$d(G)$ & 2 & 5 & 6 & 6 & 18 & 22 & 21 & 74 & 91 & 86 & 86 & 87 \\
\hline
\end{tabular}

The notation used in Tables 49 is as follows. In case $\operatorname{rk} G=n(2 \leq n \leq 4)$ we assume that $\Pi=\left\{\alpha_{1}, \alpha_{2}, \ldots, \alpha_{n}\right\}$. Column " $(\mathrm{M}, \pi)$ " contains all pairs $(\alpha, \pi(\alpha))$, where $\alpha \in \mathrm{M}$. Here, $\left(i_{1} i_{2} \ldots i_{k}, i_{j}\right)$ denotes the pair $\left(\alpha_{i_{1}}+\alpha_{i_{2}}+\cdots+\alpha_{i_{k}}, \alpha_{i_{j}}\right)$. Column " $\sim$ " indicates the equivalence classes in $\mathrm{M}$ containing more than one element. The notation $i_{1} \ldots i_{k} \sim j_{1} \ldots j_{l}$ indicates that $\alpha_{i_{1}}+\cdots+\alpha_{i_{k}} \sim \alpha_{j_{1}}+\cdots+\alpha_{j_{l}}$. Each column labeled by the type of $G$ contains all reduced sets $(\mathrm{M}, \pi, \sim)$ obtained from a given reduced set $(\mathrm{M}, \pi, \sim)$ by chains of elementary transformations in $G$. Namely, in this column, the notation $m(i)$ (resp. $m$ ) indicates that the given set $(\mathrm{M}, \pi, \sim)$ is taken to the set $(\mathrm{M}, \pi, \sim)$ in row $m$ of the same table under the elementary transformation with centre $\alpha_{i}$ (resp. under a suitable chain of elementary transformations of length greater than 1). Columns " $c(S)$ " and " $c(N)$ " contain the corresponding values. The last row of each table contains the values $d_{0}(G)$ for each of the groups $G$ considered in this table. 
TABle 4

\begin{tabular}{|c|c|c|c|c|c|c|c|}
\hline & $(\mathrm{M}, \pi)$ & $\sim$ & $A_{2}$ & $\mathrm{~B}_{2}$ & $\mathrm{G}_{2}$ & $c(S)$ & $c(N)$ \\
\hline 1 & $(12,1)$ & & $2,3(2)$ & & $3(2)$ & 0 & 2 \\
\hline 2 & $(12,2)$ & & $1,3(1)$ & $3(1)$ & & 0 & 2 \\
\hline 3 & $(1,1),(2,2)$ & & $1(2), 2(1)$ & $2(1)$ & $1(2)$ & 0 & 2 \\
\hline 4 & $(1,1),(2,2)$ & $1 \sim 2$ & & & & 1 & 1 \\
\hline \multicolumn{3}{|c|}{$d_{0}(G)$} & 2 & 3 & 3 & & \\
\hline
\end{tabular}

TABle 5

\begin{tabular}{|c|c|c|c|c|c|}
\hline & $(\mathrm{M}, \pi)$ & $\sim$ & $\mathrm{A}_{1} \times \mathrm{A}_{1} \times \mathrm{A}_{1}$ & $c(S)$ & $c(N)$ \\
\hline 1 & $(1,1),(2,2),(3,3)$ & & & 0 & 3 \\
\hline 2 & $(1,1),(2,2),(3,3)$ & $1 \sim 2$ & & 1 & 2 \\
\hline 3 & $(1,1),(2,2),(3,3)$ & $1 \sim 3$ & & 1 & 2 \\
\hline 4 & $(1,1),(2,2),(3,3)$ & $2 \sim 3$ & & 1 & 2 \\
\hline 5 & $(1,1),(2,2),(3,3)$ & $1 \sim 2 \sim 3$ & & 2 & 1 \\
\hline \multicolumn{3}{|c|}{$d_{0}(G)$} & 5 & &
\end{tabular}

TABle 6

\begin{tabular}{|c|c|c|c|c|c|c|}
\hline & $(\mathrm{M}, \pi)$ & $\sim$ & $A_{1} \times A_{2}$ & $A_{1} \times B_{2}$ & $c(S)$ & $c(N)$ \\
\hline 1 & $(1,1),(23,2)$ & & $3,5(3)$ & & 0 & 3 \\
\hline 2 & $(1,1),(23,2)$ & $1 \sim 23$ & $6(3)$ & & 1 & 2 \\
\hline 3 & $(1,1),(23,3)$ & & $1,5(2)$ & $5(2)$ & 0 & 3 \\
\hline 4 & $(1,1),(23,3)$ & $1 \sim 23$ & $7(2)$ & $7(2)$ & 1 & 2 \\
\hline 5 & $(1,1),(2,2),(3,3)$ & & $1(3), 3(2)$ & $3(2)$ & 0 & 3 \\
\hline 6 & $(1,1),(2,2),(3,3)$ & $1 \sim 2$ & $2(3)$ & & 1 & 2 \\
\hline 7 & $(1,1),(2,2),(3,3)$ & $1 \sim 3$ & $4(2)$ & $4(2)$ & 1 & 2 \\
\hline 8 & $(1,1),(2,2),(3,3)$ & $2 \sim 3$ & & & 1 & 2 \\
\hline 9 & $(1,1),(2,2),(3,3)$ & $1 \sim 2 \sim 3$ & & & 2 & 1 \\
\hline \multicolumn{3}{|c|}{$d_{0}(G)$} & 5 & 7 & & \\
\hline
\end{tabular}


TABle 7

\begin{tabular}{|c|c|c|c|c|c|c|c|}
\hline & $(\mathrm{M}, \pi)$ & $\sim$ & $\mathrm{A}_{3}$ & $\mathrm{~B}_{3}$ & $\mathrm{C}_{3}$ & $c(S)$ & $c(N)$ \\
\hline 1 & $(123,1)$ & & $3,6,8(3)$ & & $8(3)$ & 0 & 3 \\
\hline 2 & $(123,2)$ & & $4(1), 10(3), 13$ & $4(1)$ & $4(1), 10(3), 13$ & 0 & 3 \\
\hline 3 & $(123,3)$ & & $1,6(1), 8$ & $6(1), 8$ & $6(1)$ & 0 & 3 \\
\hline 4 & $(1,1),(23,2)$ & & $2(1), 10,13(3)$ & $2(1)$ & $2(1), 10,13(3)$ & 0 & 3 \\
\hline 5 & $(1,1),(23,2)$ & $1 \sim 23$ & $14(3)$ & & $14(3)$ & 1 & 2 \\
\hline 6 & $(1,1),(23,3)$ & & $1,3(1), 8(2)$ & $3(1), 8(2)$ & $3(1)$ & 0 & 3 \\
\hline 7 & $(1,1),(23,3)$ & $1 \sim 23$ & $9(2)$ & $9(2)$ & & 1 & 2 \\
\hline 8 & $(12,1),(3,3)$ & & $1(3), 3,6(2)$ & $3,6(2)$ & $1(3)$ & 0 & 3 \\
\hline 9 & $(12,1),(3,3)$ & $12 \sim 3$ & $7(2)$ & $7(2)$ & & 1 & 2 \\
\hline 10 & $(12,2),(3,3)$ & & $2(3), 4,13(1)$ & $13(1)$ & $2(3), 4,13(1)$ & 0 & 3 \\
\hline 11 & $(12,2),(3,3)$ & $12 \sim 3$ & $16(1)$ & $16(1)$ & $16(1)$ & 1 & 2 \\
\hline 12 & $(12,2),(23,2)$ & $12 \sim 23$ & & & & 1 & 2 \\
\hline 13 & $(1,1),(2,2),(3,3)$ & & $2,4(3), 10(1)$ & $10(1)$ & $2,4(3), 10(1)$ & 0 & 3 \\
\hline 14 & $(1,1),(2,2),(3,3)$ & $1 \sim 2$ & $5(3)$ & & $5(3)$ & 1 & 2 \\
\hline 15 & $(1,1),(2,2),(3,3)$ & $1 \sim 3$ & & & & 1 & 2 \\
\hline 16 & $(1,1),(2,2),(3,3)$ & $2 \sim 3$ & $11(1)$ & $11(1)$ & $11(1)$ & 1 & 2 \\
\hline 17 & $(1,1),(2,2),(3,3)$ & $1 \sim 2 \sim 3$ & & & & 2 & 1 \\
\hline \multicolumn{3}{|c|}{$d_{0}(G)$} & & 8 & 11 & 10 & \\
\cline { 1 - 5 } & & & & & \\
\hline
\end{tabular}

TABle 8

\begin{tabular}{|c|c|c|c|c|c|c|c|c|}
\hline & $(\mathrm{M}, \pi)$ & $\sim$ & $\mathrm{A}_{4}$ & $\mathrm{~B}_{4}$ & $\mathrm{C}_{4}$ & $\mathrm{~F}_{4}$ & $c(S)$ & $c(N)$ \\
\hline 1 & $(1234,1)$ & & $4,9,11(4), 19$ & & $11(4)$ & $11(4), 19$ & 0 & 4 \\
\hline 2 & $(1234,2)$ & & $\begin{array}{c}5(1), 13(4), \\
23,37,52\end{array}$ & $5(1)$ & $5(1), 13(4), 37$ & $\begin{array}{c}5(1), 13(4), \\
23,37,52\end{array}$ & 0 & 4 \\
\hline 3 & $(1234,3)$ & & $\begin{array}{c}7(1), 15(4), \\
17,27,42\end{array}$ & $7(1), 17$ & $\begin{array}{c}7(1), 15(4), \\
17,27,42\end{array}$ & $7(1), 15(4), 42$ & 0 & 4 \\
\hline 4 & $(1234,4)$ & & $1,9(1), 11,19$ & $9(1), 11,19$ & $9(1), 19$ & $9(1)$ & 0 & 4 \\
\hline 5 & $(1,1),(234,2)$ & & $\begin{array}{c}2(1), 13,23, \\
37(4), 52\end{array}$ & $2(1)$ & $2(1), 13,37(4)$ & $\begin{array}{c}2(1), 13,23, \\
37(4), 42\end{array}$ & 0 & 4 \\
\hline 6 & $(1,1),(234,2)$ & $1 \sim 234$ & $38(4), 53$ & & $38(4)$ & $38(4), 53$ & 1 & 3 \\
\hline 7 & $(1,1),(234,3)$ & & $\begin{array}{c}3(1), 15, \\
17(2), 27, \\
42(4)\end{array}$ & $3(1), 17(2)$ & $\begin{array}{c}3(1), 15, \\
17(2), 27, \\
42(4)\end{array}$ & $3(1), 15,42(4)$ & 0 & 4 \\
\hline 8 & $(1,1),(234,3)$ & $1 \sim 234$ & $\begin{array}{c}18(2), 28, \\
43(4)\end{array}$ & $18(2)$ & $\begin{array}{c}18(2), 28, \\
43(4)\end{array}$ & $43(4)$ & 1 & 3 \\
\hline 9 & $(1,1),(234,4)$ & & $\begin{array}{c}1,4(1), 11 \\
19(2)\end{array}$ & $\begin{array}{c}4(1), 11 \\
19(2)\end{array}$ & $4(1), 19(2)$ & $4(1)$ & 0 & 4 \\
\hline 10 & $(1,1),(234,4)$ & $1 \sim 234$ & $12,20(2)$ & $12,20(2)$ & $20(2)$ & & 1 & 3 \\
\hline 11 & $(123,1),(4,4)$ & & $\begin{array}{c}1(4), 4,9, \\
19(3)\end{array}$ & $4,9,19(3)$ & $1(4)$ & $1(4), 19(3)$ & 0 & 4 \\
\hline 12 & $(123,1),(4,4)$ & $123 \sim 4$ & $10,20(3)$ & $10,20(3)$ & & $20(3)$ & 1 & 3 \\
\hline 13 & $(123,2),(4,4)$ & & $\begin{array}{c}2(4), 5,23(3) \\
37(1), 52\end{array}$ & $\begin{array}{c}23(3), \\
37(1), 52\end{array}$ & $2(4), 5,37(1)$ & $\begin{array}{c}2(4), 5,23(3) \\
37(1), 52\end{array}$ & 0 & 4 \\
\hline
\end{tabular}




\begin{tabular}{|c|c|c|c|c|c|c|c|c|}
\hline & $(\mathrm{M}, \pi)$ & $\sim$ & $\mathrm{A}_{4}$ & $\mathrm{~B}_{4}$ & $\mathrm{C}_{4}$ & $\mathrm{~F}_{4}$ & $c(S)$ & $c(N)$ \\
\hline 14 & $(123,2),(4,4)$ & $123 \sim 4$ & $\begin{array}{c}24(3), 40(1) \\
55\end{array}$ & $\begin{array}{c}24(3), \\
40(1), 55\end{array}$ & $40(1)$ & $\begin{array}{c}24(3), 40(1) \\
55\end{array}$ & 1 & 3 \\
\hline 15 & $(123,3),(4,4)$ & & $\begin{array}{c}3(4), 7,17,27 \\
42(1)\end{array}$ & $27,42(1)$ & $\begin{array}{c}3(4), 7,17,27 \\
42(1)\end{array}$ & $3(4), 7,42(1)$ & 0 & 4 \\
\hline 16 & $(123,3),(4,4)$ & $123 \sim 4$ & $30,45(1)$ & $30,45(1)$ & $30,45(1)$ & $45(1)$ & 1 & 3 \\
\hline 17 & $(12,1),(34,3)$ & & $\begin{array}{c}3,7(2), 15 \\
27(4), 42\end{array}$ & $3,7(2)$ & $\begin{array}{c}3,7(2), 15 \\
27(4), 42\end{array}$ & $27(4)$ & 0 & 4 \\
\hline 18 & $(12,1),(34,3)$ & $12 \sim 34$ & $8(2), 28(4), 43$ & $8(2)$ & $8(2), 28(4), 43$ & $28(4)$ & 1 & 3 \\
\hline 19 & $(12,1),(34,4)$ & & $\begin{array}{c}1,4,9(2), \\
11(3)\end{array}$ & $\begin{array}{c}4,9(2), \\
11(3)\end{array}$ & $4,9(2)$ & $1,11(3)$ & 0 & 4 \\
\hline 20 & $(12,1),(34,4)$ & $12 \sim 34$ & $10(2), 12(3)$ & $10(2), 12(3)$ & $10(2)$ & $12(3)$ & 1 & 3 \\
\hline 21 & $(12,2),(34,3)$ & & $\begin{array}{c}32(4), 47(1) \\
61\end{array}$ & $47(1)$ & $\begin{array}{c}32(4), 47(1), \\
61\end{array}$ & $\begin{array}{c}32(4), 47(1) \\
61\end{array}$ & 0 & 4 \\
\hline 22 & $(12,2),(34,3)$ & $12 \sim 34$ & $\begin{array}{c}33(4), 50(1) \\
65\end{array}$ & $50(1)$ & $\begin{array}{c}33(4), 50(1) \\
65\end{array}$ & $\begin{array}{c}33(4), 50(1) \\
65\end{array}$ & 1 & 3 \\
\hline 23 & $(12,2),(34,4)$ & & $\begin{array}{c}2,5,13(3), 37 \\
52(1)\end{array}$ & $\begin{array}{c}13(3), 37, \\
52(1)\end{array}$ & $52(1)$ & $\begin{array}{c}2,5,13(3), 37 \\
52(1)\end{array}$ & 0 & 4 \\
\hline 24 & $(12,2),(34,4)$ & $12 \sim 34$ & $\begin{array}{c}14(3), 40 \\
55(1)\end{array}$ & $\begin{array}{c}14(3), 40, \\
55(1)\end{array}$ & $55(1)$ & $\begin{array}{c}14(3), 40 \\
55(1)\end{array}$ & 1 & 3 \\
\hline 25 & $\begin{array}{l}(12,2), \\
(234,2)\end{array}$ & $12 \sim 234$ & $57(4)$ & & $57(4)$ & $57(4)$ & 1 & 3 \\
\hline 26 & $\begin{array}{c}(123,3), \\
(34,3)\end{array}$ & $123 \sim 34$ & $59(1)$ & $59(1)$ & $59(1)$ & $59(1)$ & 1 & 3 \\
\hline 27 & $\begin{array}{c}(12,1),(3,3) \\
(4,4)\end{array}$ & & $\begin{array}{c}3,7,15,17(4) \\
42(2)\end{array}$ & $15,42(2)$ & $\begin{array}{c}3,7,15,17(4) \\
42(2)\end{array}$ & $17(4)$ & 0 & 4 \\
\hline 28 & $\begin{array}{c}(12,1),(3,3) \\
(4,4)\end{array}$ & $12 \sim 3$ & $8,18(4), 43(2)$ & $43(2)$ & $8,18(4), 43(2)$ & $18(4)$ & 1 & 3 \\
\hline 29 & $\begin{array}{c}(12,1),(3,3) \\
(4,4)\end{array}$ & $12 \sim 4$ & $44(2)$ & $44(2)$ & $44(2)$ & & 1 & 3 \\
\hline 30 & $\begin{array}{c}(12,1),(3,3) \\
(4,4)\end{array}$ & $3 \sim 4$ & $16,45(2)$ & $16,45(2)$ & $16,45(2)$ & & 1 & 3 \\
\hline 31 & $\begin{array}{c}(12,1),(3,3) \\
(4,4) \\
\end{array}$ & $12 \sim 3 \sim 4$ & $46(2)$ & $46(2)$ & $46(2)$ & & 2 & 2 \\
\hline 32 & $\begin{array}{c}(12,2),(3,3) \\
(4,4)\end{array}$ & & $\begin{array}{c}21(4), 47 \\
61(1)\end{array}$ & $61(1)$ & $\begin{array}{c}21(4), 47, \\
61(1)\end{array}$ & $\begin{array}{c}21(4), 47, \\
61(1)\end{array}$ & 0 & 4 \\
\hline 33 & $\begin{array}{c}(12,2),(3,3) \\
(4,4)\end{array}$ & $12 \sim 3$ & $\begin{array}{c}22(4), 50 \\
65(1)\end{array}$ & $65(1)$ & $\begin{array}{c}22(4), 50, \\
65(1)\end{array}$ & $\begin{array}{c}22(4), 50, \\
65(1)\end{array}$ & 1 & 3 \\
\hline 34 & $\begin{array}{c}(12,2),(3,3) \\
(4,4)\end{array}$ & $12 \sim 4$ & $66(1)$ & $66(1)$ & $66(1)$ & $66(1)$ & 1 & 3 \\
\hline 35 & $\begin{array}{c}(12,2),(3,3) \\
(4,4)\end{array}$ & $3 \sim 4$ & $67(1)$ & $67(1)$ & $67(1)$ & $67(1)$ & 1 & 3 \\
\hline 36 & $\begin{array}{c}(12,2),(3,3) \\
(4,4)\end{array}$ & $12 \sim 3 \sim 4$ & $71(1)$ & $71(1)$ & $71(1)$ & $71(1)$ & 2 & 2 \\
\hline 37 & $\begin{array}{c}(1,1),(23,2) \\
(4,4)\end{array}$ & & $\begin{array}{c}2,5(4), 13(1) \\
23,52(3)\end{array}$ & $\begin{array}{c}13(1), 23, \\
52(3)\end{array}$ & $2,5(4), 13(1)$ & $\begin{array}{c}2,5(4), 13(1) \\
23,52(3)\end{array}$ & 0 & 4 \\
\hline 38 & $\begin{array}{c}(1,1),(23,2), \\
(4,4)\end{array}$ & $1 \sim 23$ & $6(4), 53(3)$ & $53(3)$ & $6(4)$ & $6(4), 53(3)$ & 1 & 3 \\
\hline 39 & $\begin{array}{c}(1,1),(23,2) \\
(4,4)\end{array}$ & $1 \sim 4$ & $54(3)$ & $54(3)$ & & $54(3)$ & 1 & 3 \\
\hline 40 & $\begin{array}{c}(1,1),(23,2) \\
(4,4)\end{array}$ & $23 \sim 4$ & $\begin{array}{c}14(1), 24, \\
55(3)\end{array}$ & $\begin{array}{c}14(1), 24, \\
55(3)\end{array}$ & $14(1)$ & $\begin{array}{c}14(1), 24, \\
55(3)\end{array}$ & 1 & 3 \\
\hline 41 & $\begin{array}{c}(1,1),(23,2), \\
(4,4)\end{array}$ & $1 \sim 23 \sim 4$ & $56(3)$ & $56(3)$ & & $56(3)$ & 2 & 2 \\
\hline
\end{tabular}




\begin{tabular}{|c|c|c|c|c|c|c|c|c|}
\hline & $(\mathrm{M}, \pi)$ & $\sim$ & $\mathrm{A}_{4}$ & $\mathrm{~B}_{4}$ & $\mathrm{C}_{4}$ & $\mathrm{~F}_{4}$ & $c(S)$ & $c(N)$ \\
\hline 42 & $\begin{array}{c}(1,1),(23,3), \\
(4,4)\end{array}$ & & \begin{tabular}{|c|}
$3,7(4), 15(1)$ \\
$17,27(2)$
\end{tabular} & $15(1), 27(2)$ & \begin{tabular}{|c|}
$3,7(4), 15(1)$ \\
$17,27(2)$
\end{tabular} & $3,7(4), 15(1)$ & 0 & 4 \\
\hline 43 & $\begin{array}{c}(1,1),(23,3), \\
(4,4)\end{array}$ & $1 \sim 23$ & $8(4), 18,28(2)$ & $28(2)$ & $8(4), 18,28(2)$ & $8(4)$ & 1 & 3 \\
\hline 44 & $\begin{array}{c}(1,1),(23,3), \\
(4,4)\end{array}$ & $1 \sim 4$ & $29(2)$ & $29(2)$ & $29(2)$ & & 1 & 3 \\
\hline 45 & $\begin{array}{c}(1,1),(23,3), \\
(4,4)\end{array}$ & $23 \sim 4$ & $16(1), 30(2)$ & $16(1), 30(2)$ & $16(1), 30(2)$ & $16(1)$ & 1 & 3 \\
\hline 46 & $\begin{array}{c}(1,1),(23,3) \\
(4,4)\end{array}$ & $1 \sim 23 \sim 4$ & $31(2)$ & $31(2)$ & $31(2)$ & & 2 & 2 \\
\hline 47 & $\begin{array}{c}(1,1),(2,2) \\
(34,3)\end{array}$ & & $\begin{array}{c}21(1), 32 \\
61(4)\end{array}$ & $21(1)$ & $\begin{array}{c}21(1), 32, \\
61(4)\end{array}$ & $\begin{array}{c}21(1), 32 \\
61(4)\end{array}$ & 0 & 4 \\
\hline 48 & $\begin{array}{c}(1,1),(2,2) \\
(34,3)\end{array}$ & $1 \sim 2$ & $62(4)$ & & $62(4)$ & $62(4)$ & 1 & 3 \\
\hline 49 & $\begin{array}{c}(1,1),(2,2) \\
(34,3)\end{array}$ & $1 \sim 34$ & $63(4)$ & & $63(4)$ & $63(4)$ & 1 & 3 \\
\hline 50 & $\begin{array}{c}(1,1),(2,2) \\
(34,3)\end{array}$ & $2 \sim 34$ & $\begin{array}{c}22(1), 33, \\
65(4)\end{array}$ & $22(1)$ & $\begin{array}{c}22(1), 33, \\
65(4)\end{array}$ & $\begin{array}{c}22(1), 33, \\
65(4)\end{array}$ & 1 & 3 \\
\hline 51 & $\begin{array}{c}(1,1),(2,2) \\
(34,3)\end{array}$ & $1 \sim 2 \sim 34$ & $68(4)$ & & $68(4)$ & $68(4)$ & 2 & 2 \\
\hline 52 & $\begin{array}{c}(1,1),(2,2) \\
(34,4)\end{array}$ & & \begin{tabular}{|c|}
$2,5,13,23(1)$ \\
$37(3)$ \\
\end{tabular} & $\begin{array}{c}13,23(1), \\
37(3)\end{array}$ & $23(1)$ & $\begin{array}{c}2,5,13,23(1) \\
37(3) \\
\end{array}$ & 0 & 4 \\
\hline 53 & $\begin{array}{c}(1,1),(2,2) \\
(34,4)\end{array}$ & $1 \sim 2$ & $6,38(3)$ & $38(3)$ & & $6,38(3)$ & 1 & 3 \\
\hline 54 & $\begin{array}{c}(1,1),(2,2) \\
(34,4)\end{array}$ & $1 \sim 34$ & $39(3)$ & $39(3)$ & & $39(3)$ & 1 & 3 \\
\hline 55 & $\begin{array}{c}(1,1),(2,2) \\
(34,4)\end{array}$ & $2 \sim 34$ & $\begin{array}{c}14,24(1), \\
40(3)\end{array}$ & $\begin{array}{c}14,24(1), \\
40(3)\end{array}$ & $24(1)$ & $\begin{array}{c}14,24(1), \\
40(3)\end{array}$ & 1 & 3 \\
\hline 56 & $\begin{array}{c}(1,1),(2,2) \\
(34,4)\end{array}$ & $1 \sim 2 \sim 34$ & $41(3)$ & $41(3)$ & & $41(3)$ & 2 & 2 \\
\hline 57 & $\begin{array}{c}(12,2),(23, \\
2),(4,4)\end{array}$ & $12 \sim 23$ & $25(4)$ & & $25(4)$ & $25(4)$ & 1 & 3 \\
\hline 58 & $\begin{array}{c}(12,2),(23 \\
2),(4,4)\end{array}$ & $12 \sim 23 \sim 4$ & & & & & 2 & 2 \\
\hline 59 & $\begin{array}{c}(1,1),(23,3) \\
(34,3)\end{array}$ & $23 \sim 34$ & $26(1)$ & $26(1)$ & $26(1)$ & $26(1)$ & 1 & 3 \\
\hline 60 & $\begin{array}{c}(1,1),(23,3), \\
(34,3)\end{array}$ & $1 \sim 23 \sim 34$ & & & & & 2 & 2 \\
\hline 61 & $\begin{array}{c}(1,1),(2,2) \\
(3,3),(4,4)\end{array}$ & & $\begin{array}{c}21,32(1), \\
47(4)\end{array}$ & $32(1)$ & $\begin{array}{c}21,32(1), \\
47(4)\end{array}$ & $\begin{array}{c}21,32(1), \\
47(4)\end{array}$ & 0 & 4 \\
\hline 62 & $\begin{array}{c}(1,1),(2,2) \\
(3,3),(4,4)\end{array}$ & $1 \sim 2$ & $48(4)$ & & $48(4)$ & $48(4)$ & 1 & 3 \\
\hline 63 & $\begin{array}{c}(1,1),(2,2) \\
(3,3),(4,4)\end{array}$ & $1 \sim 3$ & $49(4)$ & & $49(4)$ & $49(4)$ & 1 & 3 \\
\hline 64 & $\begin{array}{c}(1,1),(2,2) \\
(3,3),(4,4)\end{array}$ & $1 \sim 4$ & & & & & 1 & 3 \\
\hline 65 & $\begin{array}{c}(1,1),(2,2) \\
(3,3),(4,4)\end{array}$ & $2 \sim 3$ & $\begin{array}{c}22,33(1), \\
50(4)\end{array}$ & $33(1)$ & $\begin{array}{c}22,33(1), \\
50(4)\end{array}$ & $\begin{array}{c}22,33(1), \\
50(4)\end{array}$ & 1 & 3 \\
\hline 66 & $\begin{array}{c}(1,1),(2,2) \\
(3,3),(4,4)\end{array}$ & $2 \sim 4$ & $34(1)$ & $34(1)$ & $34(1)$ & $34(1)$ & 1 & 3 \\
\hline 67 & $\begin{array}{c}(1,1),(2,2) \\
(3,3),(4,4)\end{array}$ & $3 \sim 4$ & $35(1)$ & $35(1)$ & $35(1)$ & $35(1)$ & 1 & 3 \\
\hline
\end{tabular}




\begin{tabular}{|c|c|c|c|c|c|c|c|c|}
\hline & $(\mathrm{M}, \pi)$ & $\sim$ & $\mathrm{A}_{4}$ & $\mathrm{~B}_{4}$ & $\mathrm{C}_{4}$ & $\mathrm{~F}_{4}$ & $c(S)$ & $c(N)$ \\
\hline 68 & $\begin{array}{c}(1,1),(2,2) \\
(3,3),(4,4)\end{array}$ & $1 \sim 2 \sim 3$ & $51(4)$ & & $51(4)$ & $51(4)$ & 2 & 2 \\
\hline 69 & $\begin{array}{c}(1,1),(2,2) \\
(3,3),(4,4)\end{array}$ & $1 \sim 2 \sim 4$ & & & & & 2 & 2 \\
\hline 70 & $\begin{array}{c}(1,1),(2,2) \\
(3,3),(4,4)\end{array}$ & $1 \sim 3 \sim 4$ & & & & & 2 & 2 \\
\hline 71 & $\begin{array}{c}(1,1),(2,2) \\
(3,3),(4,4)\end{array}$ & $2 \sim 3 \sim 4$ & $36(1)$ & $36(1)$ & $36(1)$ & $36(1)$ & 2 & 2 \\
\hline 72 & $\begin{array}{c}(1,1),(2,2) \\
(3,3),(4,4)\end{array}$ & $1 \sim 2,3 \sim 4$ & & & & & 2 & 2 \\
\hline 73 & $\begin{array}{c}(1,1),(2,2) \\
(3,3),(4,4)\end{array}$ & $1 \sim 3,2 \sim 4$ & & & & & 2 & 2 \\
\hline 74 & $\begin{array}{c}(1,1),(2,2) \\
(3,3),(4,4)\end{array}$ & $1 \sim 4,2 \sim 3$ & & & & & 2 & 2 \\
\hline 75 & $\begin{array}{c}(1,1),(2,2) \\
(3,3),(4,4)\end{array}$ & $1 \sim 2 \sim 3 \sim 4$ & & & & & 3 & 1 \\
\hline \multicolumn{3}{|c|}{$d_{0}(G)$} & 31 & 42 & 38 & 38 & & \\
\hline
\end{tabular}

TABle 9

\begin{tabular}{|c|c|c|c|c|c|}
\hline & $(\mathrm{M}, \pi)$ & $\sim$ & $\mathrm{D}_{4}$ & $c(S)$ & $c(N)$ \\
\hline 1 & $(1234,1)$ & & $11(3), 17(4), 26$ & 0 & 4 \\
\hline 2 & $(1234,2)$ & & $5(1), 13(3), 19(4), 31,36,46,63$ & 0 & 4 \\
\hline 3 & $(1234,3)$ & & $7(1), 21(4), 41$ & 0 & 4 \\
\hline 4 & $(1234,4)$ & & $9(1), 15(3), 51$ & 0 & 4 \\
\hline 5 & $(1,1),(234,2)$ & & $2(1), 13,19,31,36(4), 46(3), 63$ & 0 & 4 \\
\hline 6 & $(1,1),(234,2)$ & $1 \sim 234$ & $37(4), 47(3), 64$ & 1 & 3 \\
\hline 7 & $(1,1),(234,3)$ & & $3(1), 21,41(4)$ & 0 & 4 \\
\hline 8 & $(1,1),(234,3)$ & $1 \sim 234$ & $42(4)$ & 1 & 3 \\
\hline 9 & $(1,1),(234,4)$ & & $4(1), 15,51(3)$ & 0 & 4 \\
\hline 10 & $(1,1),(234,4)$ & $1 \sim 234$ & $52(3)$ & 1 & 3 \\
\hline 11 & $(124,1),(3,3)$ & & $1(3), 17,26(4)$ & 0 & 4 \\
\hline 12 & $(124,1),(3,3)$ & $124 \sim 3$ & $27(4)$ & 1 & 3 \\
\hline 13 & $(124,2),(3,3)$ & & $2(3), 5,19,31(4), 36,46(1), 63$ & 0 & 4 \\
\hline 14 & $(124,2),(3,3)$ & $124 \sim 3$ & $32(4), 49(1), 67$ & 1 & 3 \\
\hline 15 & $(124,4),(3,3)$ & & $4(3), 9,51(1)$ & 0 & 4 \\
\hline 16 & $(124,4),(3,3)$ & $124 \sim 3$ & $54(1)$ & 1 & 3 \\
\hline 17 & $(123,1),(4,4)$ & & $1(4), 11,26(3)$ & 0 & 4 \\
\hline 18 & $(123,1),(4,4)$ & $123 \sim 4$ & $28(3)$ & 1 & 3 \\
\hline 19 & $(123,2),(4,4)$ & & $2(4), 5,13,31(3), 36(1), 46,63$ & 0 & 4 \\
\hline 20 & $(123,2),(4,4)$ & $123 \sim 4$ & $33(3), 39(1), 68$ & 1 & 3 \\
\hline 21 & $(123,3),(4,4)$ & & $3(4), 7,41(1)$ & 0 & 4 \\
\hline 22 & $(123,3),(4,4)$ & $123 \sim 4$ & $44(1)$ & 1 & 3 \\
\hline 23 & $(123,3),(234,3)$ & $123 \sim 234$ & & 1 & 3 \\
\hline 24 & $(123,1),(124,1)$ & $123 \sim 124$ & & 1 & 3 \\
\hline & & & & & \\
\hline
\end{tabular}




\begin{tabular}{|c|c|c|c|c|c|}
\hline & $(\mathrm{M}, \pi)$ & $\sim$ & $\mathrm{D}_{4}$ & $c(S)$ & $c(N)$ \\
\hline 25 & $(234,4),(124,4)$ & $234 \sim 124$ & & 1 & 3 \\
\hline 26 & $(12,1),(3,3),(4,4)$ & & $1,11(4), 17(3)$ & 0 & 4 \\
\hline 27 & $(12,1),(3,3),(4,4)$ & $12 \sim 3$ & $12(4)$ & 1 & 3 \\
\hline 28 & $(12,1),(3,3),(4,4)$ & $12 \sim 4$ & $18(3)$ & 1 & 3 \\
\hline 29 & $(12,1),(3,3),(4,4)$ & $3 \sim 4$ & & 1 & 3 \\
\hline 30 & $(12,1),(3,3),(4,4)$ & $12 \sim 3 \sim 4$ & & 2 & 2 \\
\hline 31 & $(12,2),(3,3),(4,4)$ & & $2,5,13(4), 19(3), 36,46,63(1)$ & 0 & 4 \\
\hline 32 & $(12,2),(3,3),(4,4)$ & $12 \sim 3$ & $14(4), 49,67(1)$ & 1 & 3 \\
\hline 33 & $(12,2),(3,3),(4,4)$ & $12 \sim 4$ & $20(3), 39,68(1)$ & 1 & 3 \\
\hline 34 & $(12,2),(3,3),(4,4)$ & $3 \sim 4$ & $69(1)$ & 1 & 3 \\
\hline 35 & $(12,2),(3,3),(4,4)$ & $12 \sim 3 \sim 4$ & $73(1)$ & 2 & 2 \\
\hline 36 & $(1,1),(23,2),(4,4)$ & & $2,5(4), 13,19(1), 31,46,63(3)$ & 0 & 4 \\
\hline 37 & $(1,1),(23,2),(4,4)$ & $1 \sim 23$ & $6(4), 47,64(3)$ & 1 & 3 \\
\hline 38 & $(1,1),(23,2),(4,4)$ & $1 \sim 4$ & $66(3)$ & 1 & 3 \\
\hline 39 & $(1,1),(23,2),(4,4)$ & $23 \sim 4$ & $20(1), 33,68(3)$ & 1 & 3 \\
\hline 40 & $(1,1),(23,2),(4,4)$ & $1 \sim 23 \sim 4$ & $71(3)$ & 2 & 2 \\
\hline 41 & $(1,1),(23,3),(4,4)$ & & $3,7(4), 21(1)$ & 0 & 4 \\
\hline 42 & $(1,1),(23,3),(4,4)$ & $1 \sim 23$ & $8(4)$ & 1 & 3 \\
\hline 43 & $(1,1),(23,3),(4,4)$ & $1 \sim 4$ & & 1 & 3 \\
\hline 44 & $(1,1),(23,3),(4,4)$ & $23 \sim 4$ & $22(1)$ & 1 & 3 \\
\hline 45 & $(1,1),(23,3),(4,4)$ & $1 \sim 23 \sim 4$ & & 2 & 2 \\
\hline 46 & $(1,1),(24,2),(3,3)$ & & $2,5(3), 13(1), 19,31,36,63(4)$ & 0 & 4 \\
\hline 47 & $(1,1),(24,2),(3,3)$ & $1 \sim 24$ & $6(3), 37,64(4)$ & 1 & 3 \\
\hline 48 & $(1,1),(24,2),(3,3)$ & $1 \sim 3$ & $65(4)$ & 1 & 3 \\
\hline 49 & $(1,1),(24,2),(3,3)$ & $24 \sim 3$ & $14(1), 32,67(4)$ & 1 & 3 \\
\hline 50 & $(1,1),(24,2),(3,3)$ & $1 \sim 24 \sim 3$ & $70(4)$ & 2 & 2 \\
\hline 51 & $(1,1),(24,4),(3,3)$ & & $4,9(3), 15(1)$ & 0 & 4 \\
\hline 52 & $(1,1),(24,4),(3,3)$ & $1 \sim 24$ & $10(3)$ & 1 & 3 \\
\hline 53 & $(1,1),(24,4),(3,3)$ & $1 \sim 3$ & & 1 & 3 \\
\hline 54 & $(1,1),(24,4),(3,3)$ & $24 \sim 3$ & $16(1)$ & 1 & 3 \\
\hline 55 & $(1,1),(24,4),(3,3)$ & $1 \sim 24 \sim 3$ & & 2 & 2 \\
\hline 56 & $(1,1),(23,2),(24,2)$ & $23 \sim 24$ & & 1 & 3 \\
\hline 57 & $(1,1),(23,2),(24,2)$ & $1 \sim 23 \sim 24$ & & 2 & 2 \\
\hline 58 & $(12,2),(24,2),(3,3)$ & $12 \sim 24$ & & 1 & 3 \\
\hline 59 & $(12,2),(24,2),(3,3)$ & $12 \sim 24 \sim 3$ & & 2 & 2 \\
\hline 60 & $(12,2),(23,2),(4,4)$ & $12 \sim 23$ & & 1 & 3 \\
\hline 61 & $(12,2),(23,2),(4,4)$ & $12 \sim 23 \sim 4$ & & 2 & 2 \\
\hline 62 & $(12,2),(23,2),(24,2)$ & $12 \sim 23 \sim 24$ & & 2 & 2 \\
\hline 63 & $(1,1),(2,2),(3,3),(4,4)$ & & $2,5,13,19,31(1), 36(3), 46(4)$ & 0 & 4 \\
\hline 64 & $(1,1),(2,2),(3,3),(4,4)$ & $1 \sim 2$ & $6,37(3), 47(4)$ & 1 & 3 \\
\hline
\end{tabular}




\begin{tabular}{|c|c|c|c|c|c|}
\hline & $(\mathrm{M}, \pi)$ & $\sim$ & $\mathrm{D}_{4}$ & $c(S)$ & $c(N)$ \\
\hline 65 & $(1,1),(2,2),(3,3),(4,4)$ & $1 \sim 3$ & $48(4)$ & 1 & 3 \\
\hline 66 & $(1,1),(2,2),(3,3),(4,4)$ & $1 \sim 4$ & $38(3)$ & 1 & 3 \\
\hline 67 & $(1,1),(2,2),(3,3),(4,4)$ & $2 \sim 3$ & $14,32(1), 49(4)$ & 1 & 3 \\
\hline 68 & $(1,1),(2,2),(3,3),(4,4)$ & $2 \sim 4$ & $20,33(1), 39(3)$ & 1 & 3 \\
\hline 69 & $(1,1),(2,2),(3,3),(4,4)$ & $3 \sim 4$ & $34(1)$ & 1 & 3 \\
\hline 70 & $(1,1),(2,2),(3,3),(4,4)$ & $1 \sim 2 \sim 3$ & $50(4)$ & 2 & 2 \\
\hline 71 & $(1,1),(2,2),(3,3),(4,4)$ & $1 \sim 2 \sim 4$ & $40(3)$ & 2 & 2 \\
\hline 72 & $(1,1),(2,2),(3,3),(4,4)$ & $1 \sim 3 \sim 4$ & & 2 & 2 \\
\hline 73 & $(1,1),(2,2),(3,3),(4,4)$ & $2 \sim 3 \sim 4$ & $35(1)$ & 2 & 2 \\
\hline 74 & $(1,1),(2,2),(3,3),(4,4)$ & $1 \sim 2,3 \sim 4$ & & 2 & 2 \\
\hline 75 & $(1,1),(2,2),(3,3),(4,4)$ & $1 \sim 3,2 \sim 4$ & & 2 & 2 \\
\hline 76 & $(1,1),(2,2),(3,3),(4,4)$ & $1 \sim 4,2 \sim 3$ & & 3 & 2 \\
\hline 77 & $(1,1),(2,2),(3,3),(4,4)$ & $1 \sim 2 \sim 3 \sim 4$ & & & \\
\hline \multicolumn{7}{|r|}{$d_{0}(G)$} & & & \\
\hline
\end{tabular}

\section{ACKNOWLEDGEMENTS}

The author thanks N.E. Gorfinkel' for numerous conversations which stimulated the development of the active root theory, and also È. B. Vinberg for useful discussions.

\section{REFERENCES}

[1] M. Krämer, Sphärische Untergruppen in kompakten zusammenhängenden Liegruppen, Compositio Math. 38 (1979), no. 2, 129-153. MR.528837 (80f:22011)

[2] I. V. Mikityuk, Integrability of invariant Hamiltonian systems with homogeneous configuration spaces, Mat. Sb. 129 (1986), no. 4, 514-534; English transl., Math. USSR-Sb. 57 (1987), 527-546. MR $842398(88 \mathrm{e}: 58032)$

[3] M. Brion, Classification des espaces homogènes sphériques, Compositio Math. 63 (1987), no. 2, 189-208. MR 906369 (89d:32068)

[4] O. S. Yakimova, Weakly symmetric spaces of semisimple Lie groups, Vestnik Moskov. Univ. Ser. I Mat. Mekh. 2002 (2002), no. 2, 57-60; English transl., Moscow Univ. Math. Bull. 57 (2002), no. 2, 37-40. MR:1934062 (2004b:53076)

[5] D. Luna, Sous-groupes sphériques résolubles, Prépublication de l'Institut Fourier, 1993, no. 241.

[6] D. Luna, Variétés sphériques de type A, IHÉS Publ. Math. 94 (2001), 161-226. MR1896179 (2003f:14056)

[7] P. Bravi and G. Pezzini, Wonderful varieties of type $B$ and $C$, arXiv:0909.3771v1.

[8] S. Cupit-Foutou, Wonderful varieties: a geometrical realization, arXiv:0907.2852v3.

[9] R. Avdeev, On solvable spherical subgroups of semisimple algebraic groups, Oberwolfach Reports, 7 (2010), no. 2, 1105-1108.

[10] È. B. Vinberg and A. L. Onishchik, Seminar on Lie groups and algebraic groups, URSS, Moscow, 1995; English transl. of 1st ed., A. L. Onishchik and È. B. Vinberg, Lie groups and algebraic groups, Springer Series in Soviet Mathematics, Springer-Verlag, Berlin, 1990. MR.1403378 (97d:22001)

[11] P.-L. Montagard, Une nouvelle propriété de stabilité du pléthysme, Comment. Math. Helvetici, 71 (1996), 475-505. MR1418950 (97m:20054)

[12] È. B. Vinberg, Commutative homogeneous spaces and co-isotropic symplectic actions, Uspekhi Mat. Nauk 56 (2001), no. 1, 3-62; English transl., Russian Math. Surveys 56 (2001), no. 1, 1-60. MR.1845642(2002f:53088)

Moscow State University, Russia

E-mail address: suselr@yandex.ru

Translated by E. KHUKHRO

Translation edited by the author 